\title{
DESIGN MANUAL
}

\author{
Submitted to \\ The Department of Energy
}

By

Thomas E. Burch

Paut D. Chancellor

David F. Dyer

Glennon Maples

\author{
High Temperature Heat Pump \\ Contract \# DE-ACO1-79CS-40163 \\ Mechanical Engineering Department \\ Auburn University \\ Auburn, Alabama \\ January 1980
}




\section{DISCLAIMER}

This report was prepared as an account of work sponsored by an agency of the United States Government. Neither the United States Government nor any agency Thereof, nor any of their employees, makes any warranty, express or implied, or assumes any legal liability or responsibility for the accuracy, completeness, or usefulness of any information, apparatus, product, or process disclosed, or represents that its use would not infringe privately owned rights. Reference herein to any specific commercial product, process, or service by trade name, trademark, manufacturer, or otherwise does not necessarily constitute or imply its endorsement, recommendation, or favoring by the United States Government or any agency thereof. The views and opinions of authors expressed herein do not necessarily state or reflect those of the United States Government or any agency thereof. 


\section{DISCLAIMER}

Portions of this document may be illegible in electronic image products. Images are produced from the best available original document. 


\section{DESIGN MANUAL}

Submitted to
The Department of Energy

By

Thomas E. Burch

Paul D. Chancellor

David F. Dyer

Glennon Maples

High Temperature Heât Pump
Contract \# DE-ACOT-79CS-40163

Mechanical Engineering Department Auburn University Auburn, Alabama

January 1980

This book was prepared as an accounis ot work stwunsorod by an mpency of the United States Government. Neither the United States Government nor any agency thereof, hor any of their employeets, nivekcc any completeness, or usefuness of any intormation, apparatus, product, or process disclosed. a represents that its use would not intringe privately owmed sights. Reterences herein to any specific commmercial product, processs, or service by trade nante. tradenterk, manulacturet, or otherwise, does

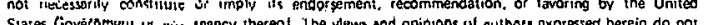

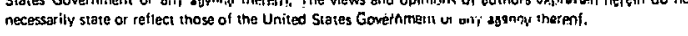


TABLE OF CONTENTS

CHAPTER 1: INTRODUCTION AND JUSTIFICATION . . . . . . . . . . 1.1

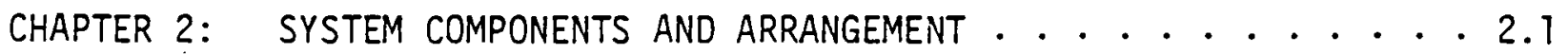

A. Electric Motor. . . . . . . . . . 2.7

1. Applications

2. Possible Configurations

3. Best System Design

4. Advantages and Disadvantages

B. Steam Turbine . . . . . . . . . . 2.8

1. Applications

2. Possible Configurations

3. Advantages and Disadvantages

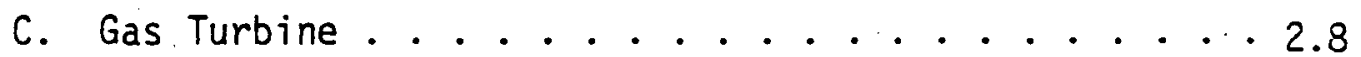

1. Applications

2. Possible Configurations

3. Advantages and Disadvantages

CHAPTER 3: SYSTEM ANALYSIS FOR ELECTRIC MOTOR . . . . . . . . . . . . 3.1

A. Analysis

B. Computer Programming

CHAPTER 4: OPTIMIZATION STUDY FOR ELECTRIC MOTOR . . . . . . 4.1

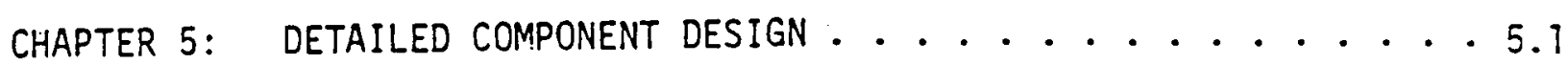

A. The Compressor .............. 5.2

B. The Heat Exchangers .......... . . 5.15

C. The Accumulator Tanks . . . . . . . . . 5.23

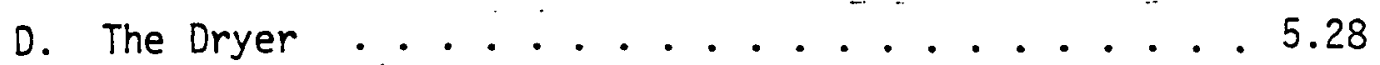




\section{TABLE OF CONTENTS(CONT'D)}

CHAPTER 6: THE CONTROL SYSTEM . . . . . . . . . . . 6.1

CHAPTER 7: LAYOUT AND CONSTRUCTION. . . . . . . . . 7.1

CHAPTER 8: ECONOMICS AND SUMMARY . . . . . . . . . . 8.1 


\section{Chapter 1}

\section{INTRODUCTION AND JUSTIFICATION}

The purpose of this final design report is to submit the work carried out under the initial research phase (PHASE I) of the DOE Contract No. DE-AC01-79CS40163. Current funding is for PHASE I only.

The potential exists in industry today to save large quantities of energy through process modification and recovery from waste energy streams. Application of current technology and initiation of research to begin and sustain increased energy savings is the bottom line in maintaining this country's high standard of living while limiting its dependence on imported energy products. The work detailed describes a waste heat recovery system utilizing a high temperature heat pump and intended for use in those industries incorporating indirect drying processes. The industrial segments which could achieve significant savings from this waste heat recovery system are diverse and include such energy intensive members as the pulp and paper industry and the textile industry. These industries presently funnel up to 60 percent of their total energy flow into indirect drying processes and make little to no attempt to recapture and reuse energy in the exhaust streams. Conservative estimates show that up to 30 percent of this otherwise wasted energy can be reclaimed for use in the process. Application of this waste heat recovery system in the pulp, paper, and textile industries has the potential to save $3.9 \times 10^{14}$ BTU/year or some $7.8 \times 10^{8}$ dollars/year in gas dollar equivalents (assuming a fuel cost of $\$ 2 /$ million BTU).

The outstanding potential for saving energy is realized by many manufacturers in the paper, pulp, and textile business, but they are wary of the initial 
capitol investment and even more concerned about the technological difficulties of an as yet unproved system. It seems that most manufacturers have adopted a wait and see attitude despite ever-rising energy costs, preferring to retain methods which have been utilized for the past 50 years. It becomes evident that the realization of these savings on a large scale depends on the acceptance of high temperature heat pump recovery systems on the marketplace, based on a documented record of successful operation. The intentions of this research and development project are thus to first design a prototype system; second, construct the prototype system and gather operational data from $i t$; and finally, to place a fully sized system in industry to prove its ability to operate successfulty and reliably in that environment. 
Chapter 2

\section{SYSTEM COMPONENTS AND ARRANGEMENT}

\section{General Requirements}

The vapor compression heat pump cycle inherently posesses four basic pieces of equipment: 1) an evaporator which transfers heat from the heat source to the refrigerant, 2) a compressor which raises the pressure and temperature of the refrigerant, 3) a condenser. which transfers heat from the refrigerant to the medium to be heated, and 4) an expansion valve or restriction which reduces the pressure and temperature to allow evaporation. The introduction of methanol as a working fluid along with the high temperature application being considered places more demanding requirements on the system's hardware than those found in more conventional systems.

The design temperatures of the evaporator and condenser are $175^{\circ} \mathrm{F}$ and $330^{\circ} \mathrm{F}$ respertively. Using these saturation temperatures the saturation pressures were found to be 25.5 PSIA at $175^{\circ} \mathrm{F}$ and 284 PSIA at $330^{\circ} \mathrm{F}$. This yields a compressor pressure ratio of 11.1 to 1 . Since the maximum practical pressure ratio of a centrifugal or axidl flow compressor is below this value (approximately 7:1) a two stage compressor is required. This is shown in Figure 2.1 .

Methanol is a dry compressing fluid. This means that as methanol vapor is compressed it moves away from the saturation dome into the superheat region. This superheating effect causes a reduced fiuid density which requires more work for each successive increment of pressure rise. This effect ean be controlled by forcing the vapor to remain close to the saturation dome through inner-conling. Multistage compression readily 


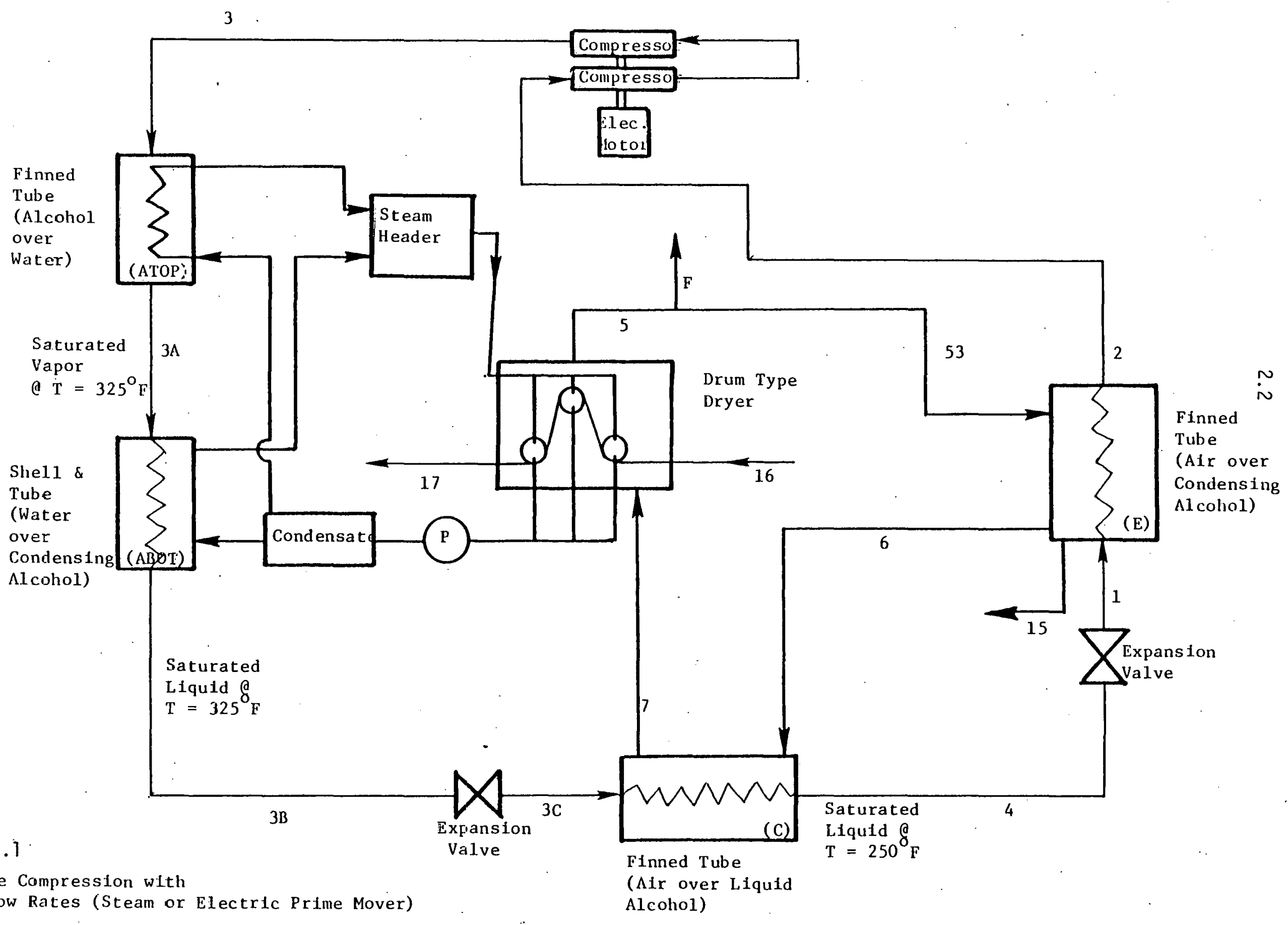

\section{FIGURE 2.1}

Two Stage Compression with

Equal Flow Rates (Steam or Electric Prime Mover)
(Air over Liquid

Alcohol)
N

(n)

Tube

ondensing

Al cohol) 
lends itself to inner-cooling and has already been deemed necessary for this application. The desired inner-cooling can be achieved by any one of several methods. One possible method is to pass the superheated refrigerant through a coil placed in cool water as shown in Figure 2.2. Although this method will work, it is undesirable because part of the energy of the working fluid is lost. It is also difficult to assure that the state of the refrigerant leaving the coil and entering the second stage of compression is a saturated vapor instead of a superheated vapor or a mixture of liquid and vapor. Another possibility would be to desuperheat the refrigerant by preheating the air entering the dryer. This would recover the energy from the refrigerant but requires the use of an expensive gas to air heat exchanger and does no:t solve the control problem. A better solution would be to bubble the superheated vapor through a saturated liquild at the same pressure as shown in Figure 2.3. This system would recover the energy of the superheated vapor (by increasing the mass flow rate of saturated vapor) while giving more positive control over the state of the refrigerant enlering the second stage of compression. The - refrigerant removed from the accumulator tank is returned to the tank from Heat Exchanger $C$ as a saturated liquid at the same temperature. Notice in Figure 2.3 that three separate heat exchangers are used in removing heat from the methanol, two of which produce steam. This seemed necessary at first due to the superheated state of the methanol at the compressor exit. Superheated vapors have poor heat transfer coefficients requiring a finned tube heat exchanger to provide the necessary surface area at a reasonable cost. Heat Exchanger ATOP is a finned tube heat 


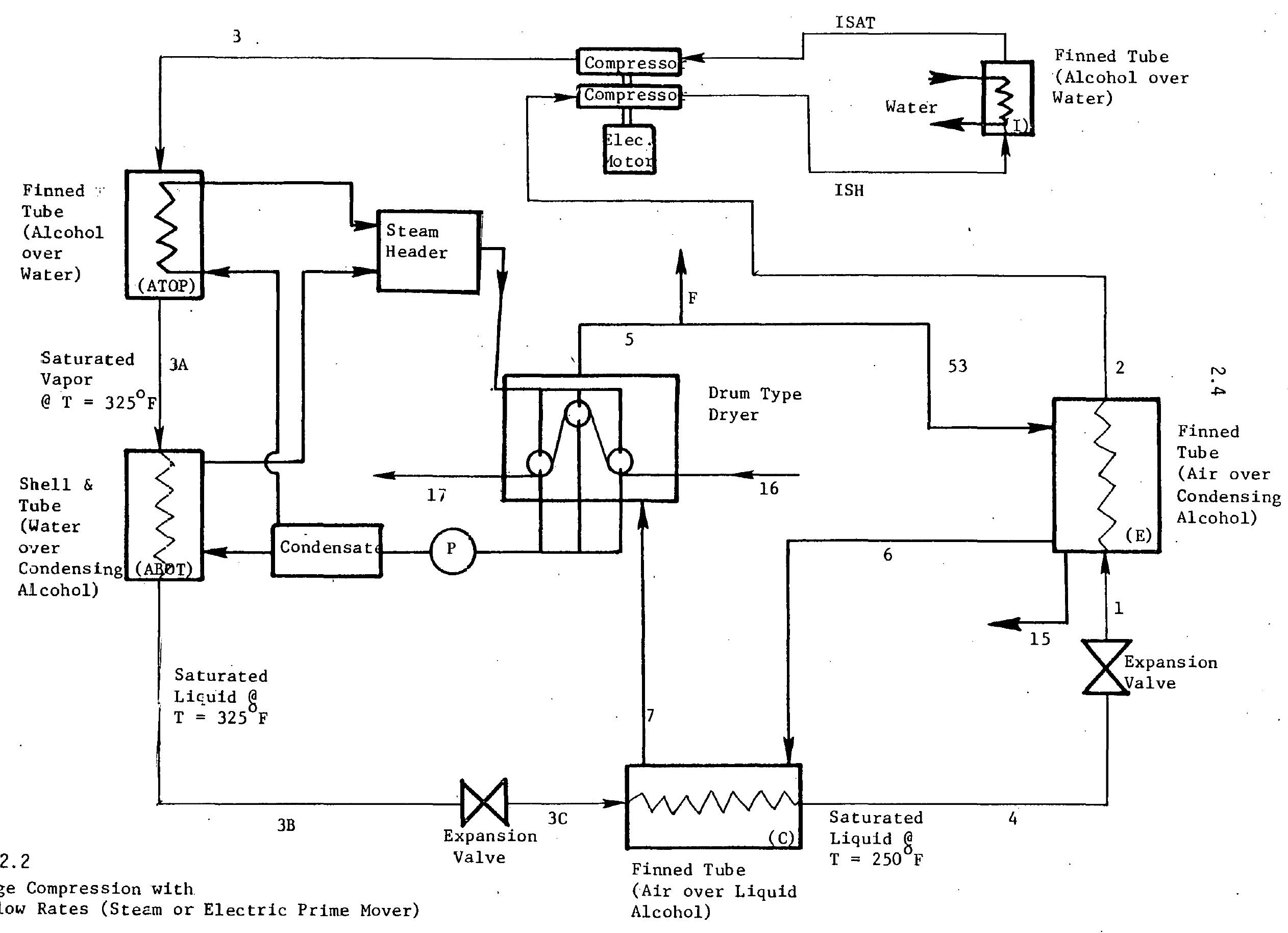

FIGURE 2.2

Two Stage Compression with

Equal Flow Rates (Steem or Electric Prime Mover)
(Air over Liquid

Alcohol) 


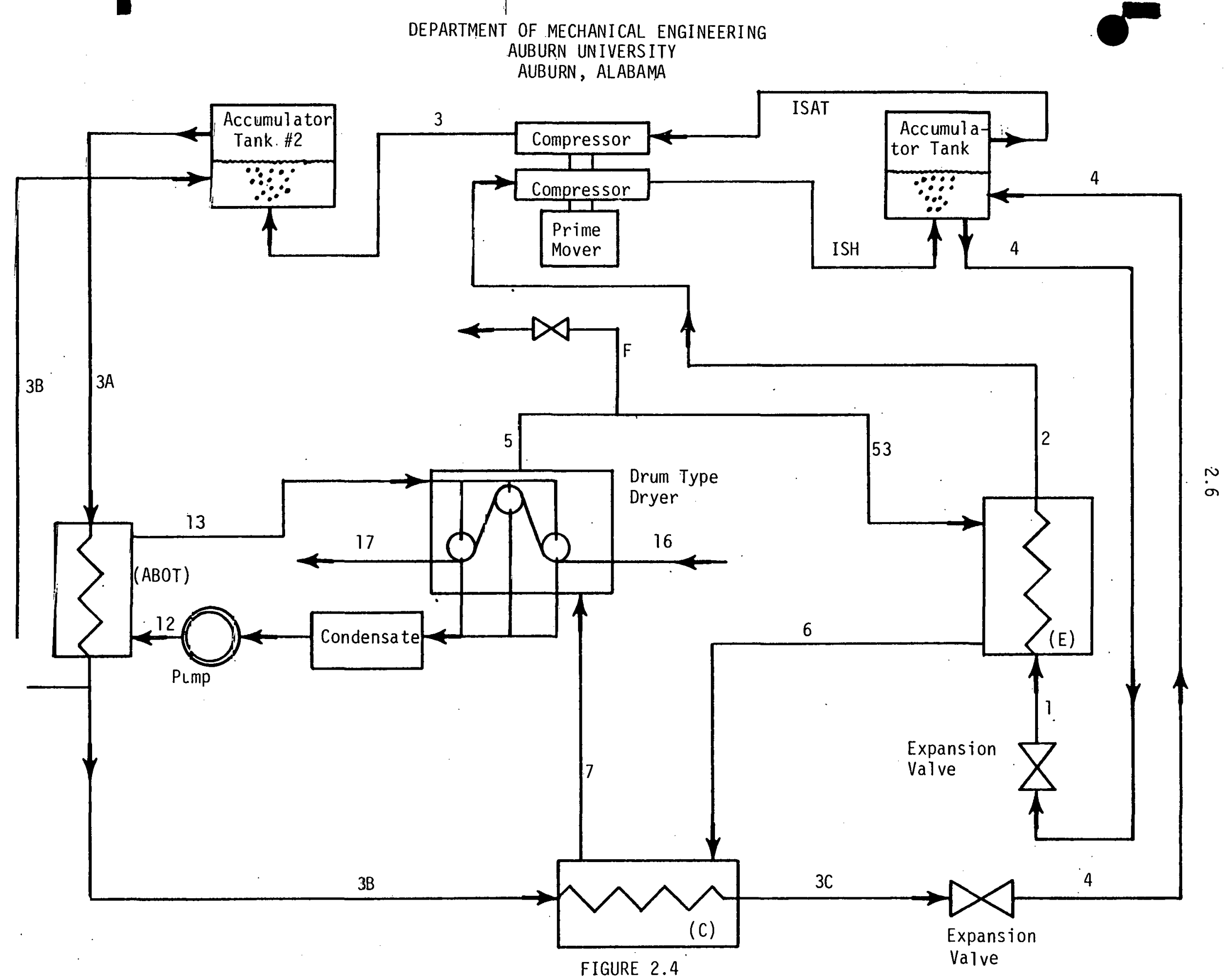




\section{7}

exchanger used to de-superheat the methanol vapor. By applying the same technique used to innercool the vapor between compression stages, Heat Exchanger ATOP was replaced by an accumulator tank (shown in Figure 2.4). Once again no energy is lost and more positive control is gained with a reduced cost. The liquid level in this tank is maintained by feeding part of the condensed riquid at State $3 B$ back into the tank. The bubbling process will also serve as a final separator to remove any oil that might be entrained in the refrigerant stream.: This is important because the oil would have a tendency to precipitate or plate out in the condenser, fouling the tubes.

Several prime movers are available for providing power to the compressor each having characteristics appropriate for a particular application. The following pages contain a discussion of some conventional prime movers with suitable applications and their effects on system design and performance.

\section{Electric Motor}

The electric motor is probabiy the most versatile and certainly the most widely used prime mover available. Electric motors are preferred by many industrtal consumers because of their ready availability and relatively low initial cost. Ease of operation, low maintenance and the availability of electricity are also attractive features. The electric motor is the unanimous choice for most small applications and is very competitive for power requirements not exceeding 2000 horsepower. For most larger applications the cost of operation (primarily cost of electricity) becomes a dominant factor and other prime movers are chosen. 
For the high temperature heat pump demonstration unit, the electric motor was chosen for economic reasons and because compressor power requirements can be easily and accurately measured. Figure 2.5 shows a sketch of the demonstration unit utilizing an electric motor. No system modifications or extra equipment are necessary for this prime mover.

\section{Steam Turbine}

For applications where large quantities of high pressure steam are available, the steam turbine may be the best choice, especially where steam is generated from waste fired boilers. The initial cost of a steam turbine is high (usually from two to five times that of an electric motor of equal output). The operating cost, however, can be significantly lower. If low pressure process steam is required, a non-condensing steam turbine can be effectively used. The turbine can be used in the place of a pressure reducing valve with the turbine exhaust steam going to the low pressure application. With these conditions, the initial cost of a steam turbine can easily be justlfied. A schematic of the heat pump system with steam turbine drive is shown in Figure 2.6. Note that no modification of system components or design is necessary.

\section{Gas Turbine or Diesel}

The use of gas turbines as prime movers is primarily restricted to large applications. Although the gas turbine driven heat pump would have the best overall energy efficiency of the four prime movers considered, the purchase and installation costs are prohibitive. The use of gas turbine drive would have its greatest advantage in applications requiring 2,000 or more horsepower. For these large horsepower units, the equipment cost in dollars per horsepower would be more competitive. 


\section{FIGURE 2.5 HEAT PUMP WASTE HEAT RECOVERY SYSTEM}

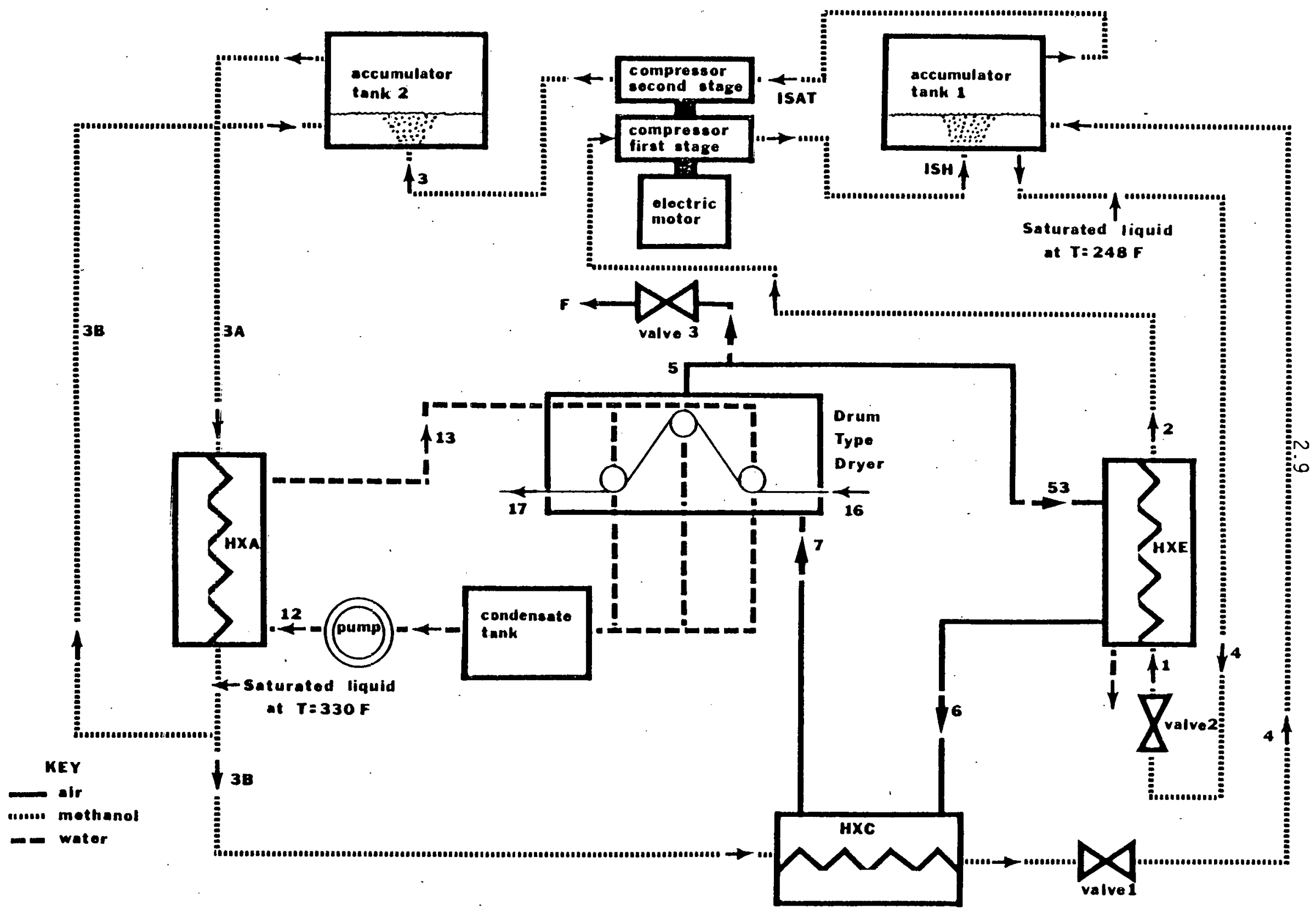




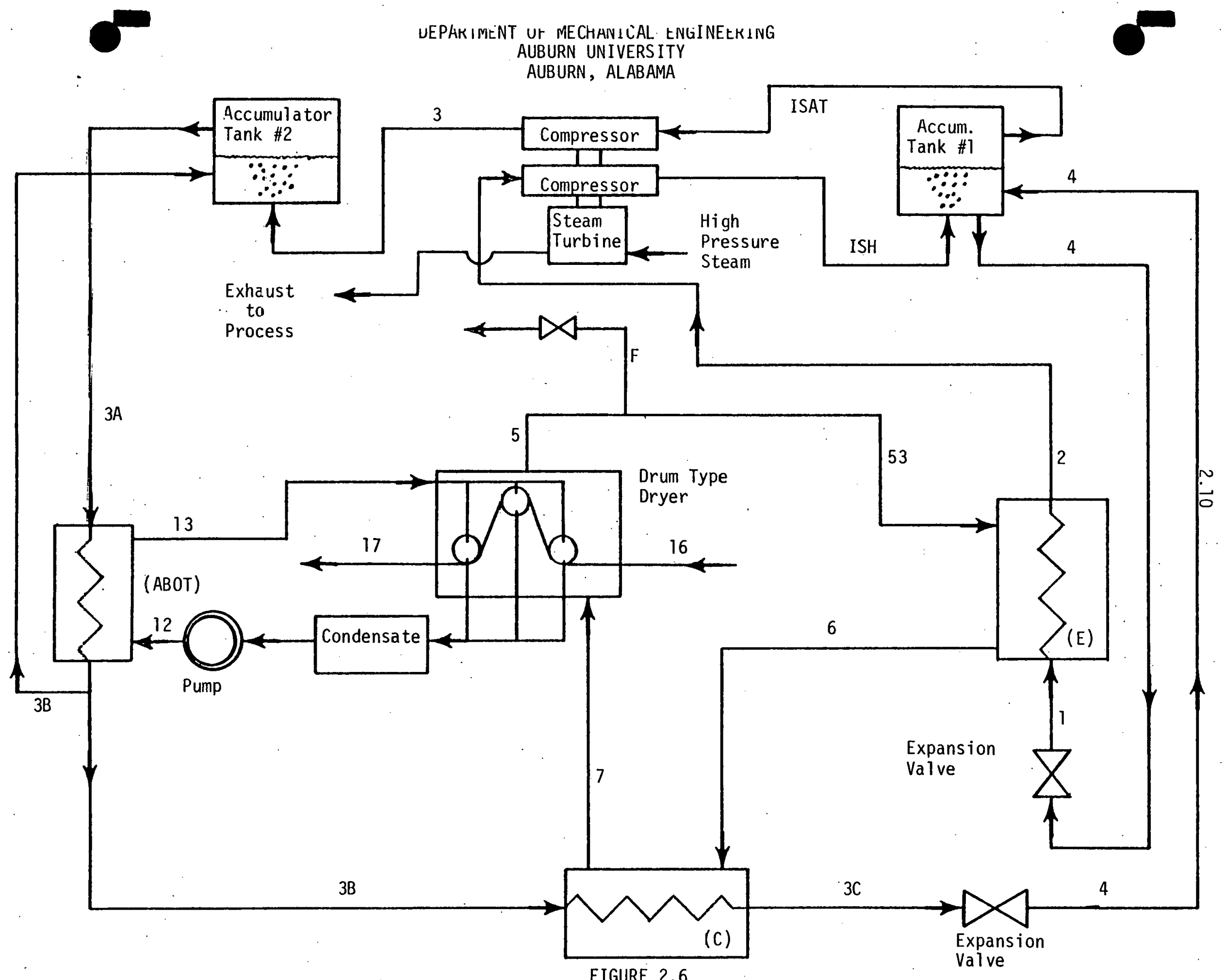


Diesel engines are available in virtually any power range required. The overall efficiency of a diesel powered heat pump would be very good, approaching that of a gas turbine. The initial cost of a diesel in dollars per horsepower lies somewhere between those of an electric motor and a gas turbine. The diesel engine also provides a power source independent of electric power as does the gas turbine. The major disadvantages of the diesel are high maintenance cost and comparatively short life.

The key to the high efficiency of either the gas turbine or diesel drive lies in the utilization of prime mover exhaust heat. This energy can be used to produce part of the steam required for drying or to produce extra steam to be used elsewhere in the plant. If the steam can be used in other 'processes, the unit will be able to recover and recycle more of the energy used for drying thus minimizing waste and increasing system efficiency. Figure 2.7 shows a schematic of the system producing extra steam. If the system must be self contained (i.e., no extra steam produced), there are two options available. The prime mover exhaust can be discharged directly to the atmosphere (shown in Figure 2.8) carrying with it one third to one half of the total energy available in the fuel used. The other option would be to recover the available exhaust energy and produce steam for the drying process. For this case, the size of the prime mover, compressor and heat exchangers would be reduced along with their initial cost. Figure 2.9 illustrates a self-contained heat pump system utilizing available exhaust energy.

Note in Figure 2.7 and Figure 2.9 that a waste heat boiler or heat exchanger is necessary for exhaust heat recovery.

The appropriate choice of prime movers for any industrial application would be highly dependent on the industrial process involved. Before a choice is made, an analysis of industry requirements and system compatability 


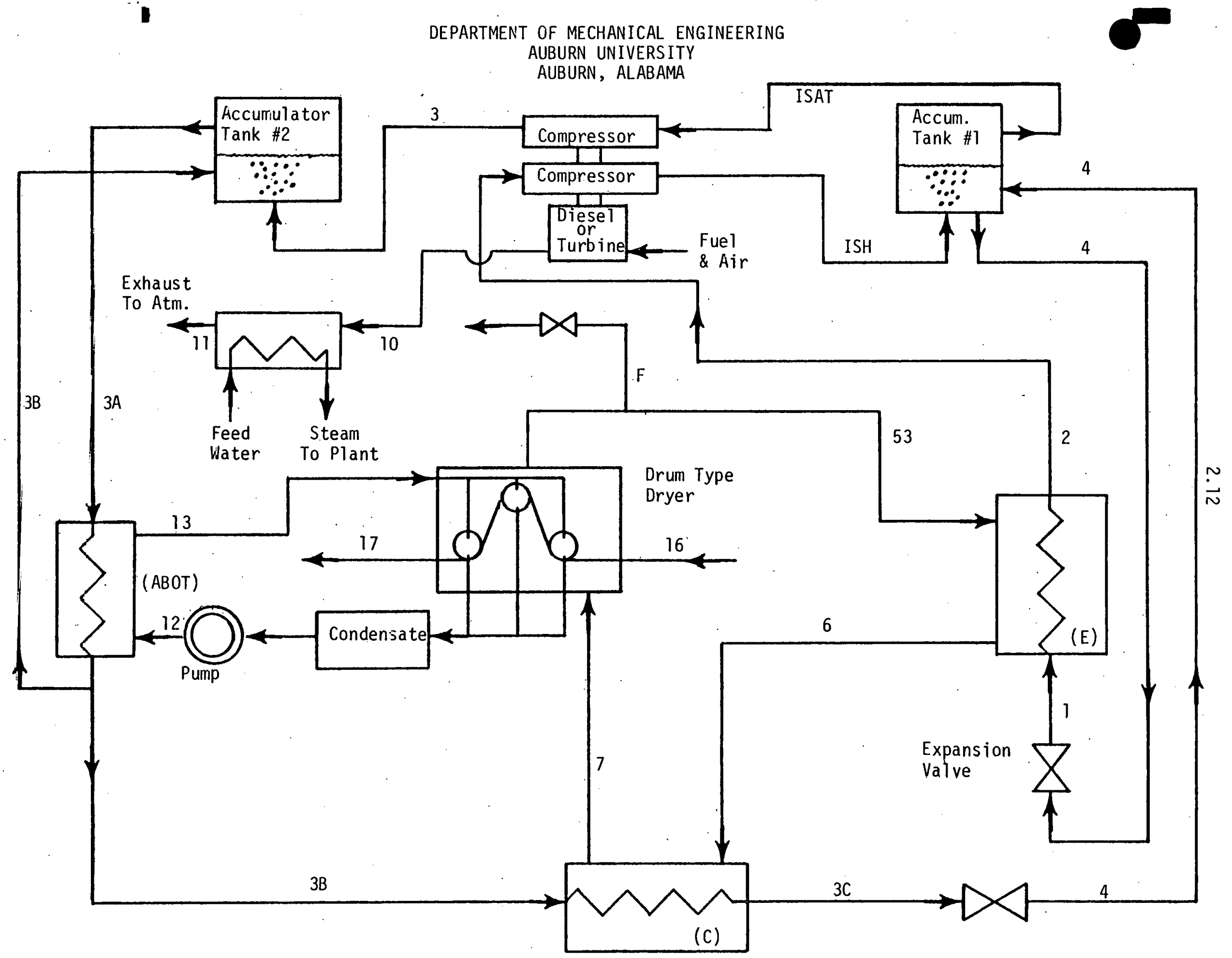

FIGURE 2.7 


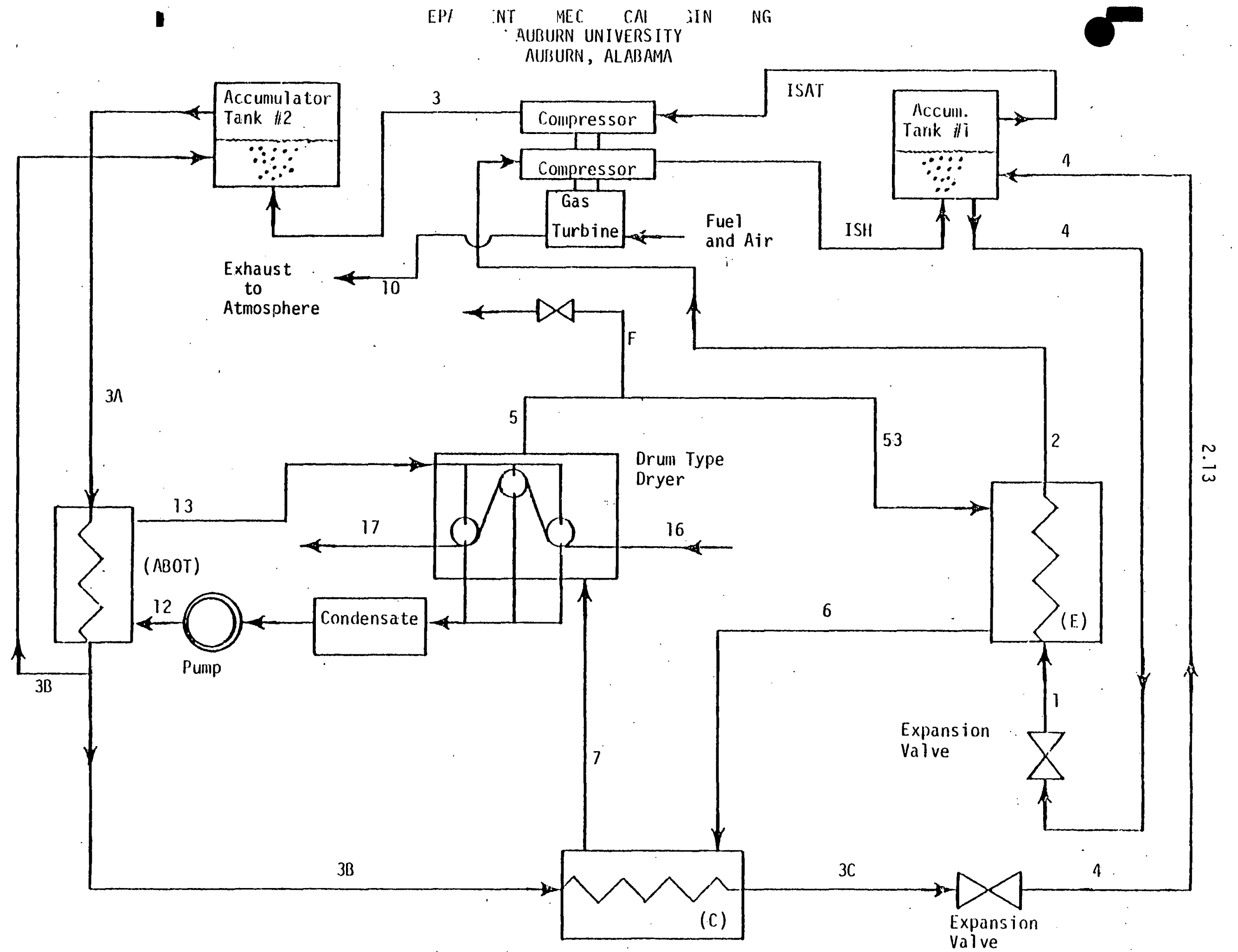

FIrIIRF ? $R$ 
with existing equipment and facilities should be completed. From this analysis, along with an economic evaluation of system performance, a prime mover and system arrangement can be selected to give desirable results for virtually any application. 


\section{SYSTEM ANALYSIS}

The selection of components to be used and the optimum arrangement of these components in the system is presented in Section 2 of this manual. The purpose of this section on system analysis is to give a step by step procedure for analyzing the performance of this system for any given set of operating conditions. The analysis is then presented in computer language (FORTRAN) which provides the designer with a useful tool for predicting system performance for a wide range of system variables. With this information in hand a set of system variables can be chosen which will give optimum performance and practicality for a particular application.

The system to be discussed here is shown in Figure 3.1 with all points labled and defined in Table 3.1. 


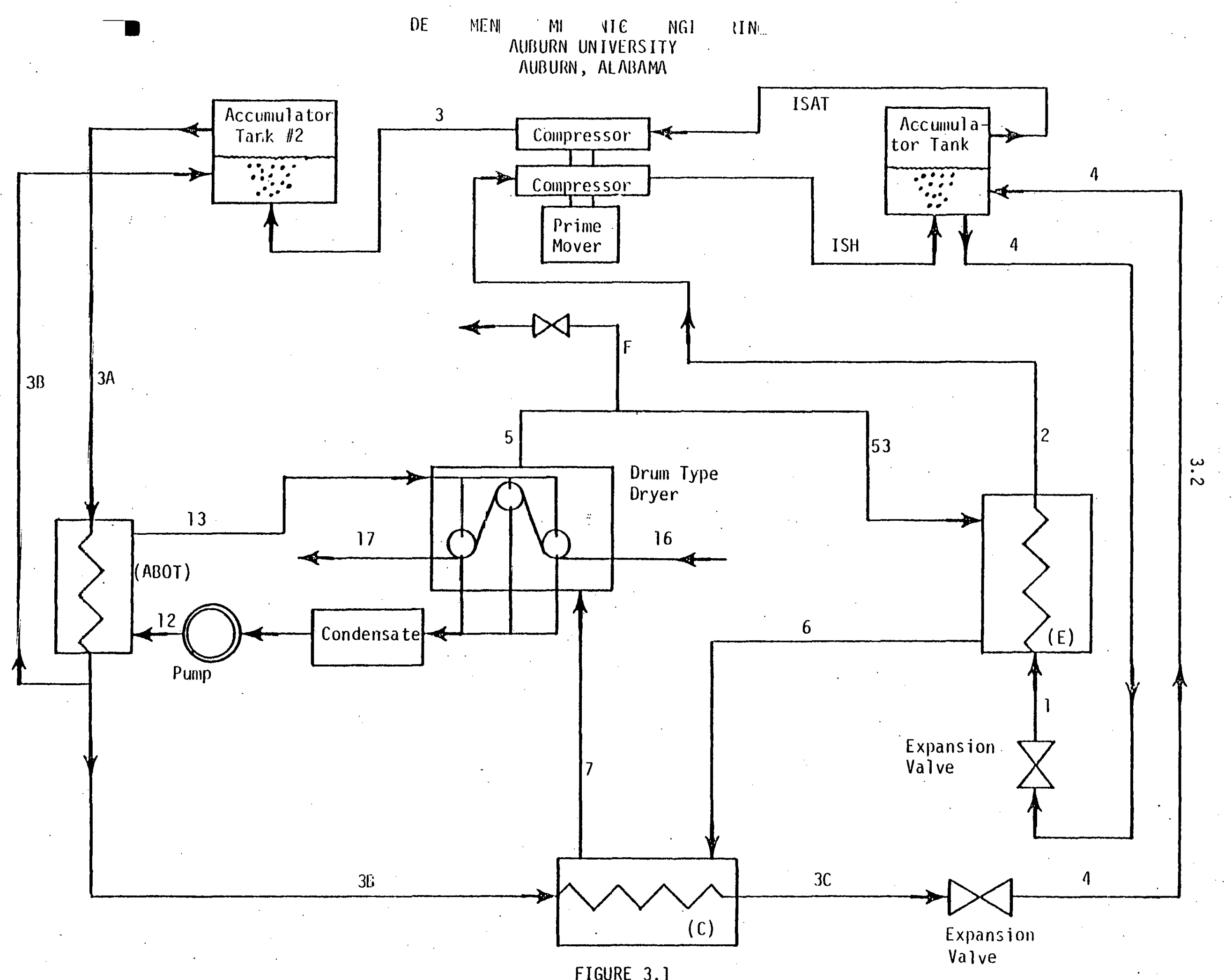


TABLE 3.1

Refrigerant Loop:

\begin{tabular}{|c|c|c|}
\hline State 1 & - & $\begin{array}{l}\text { Saturated Mixture of Liquid and Vapor at Temperature } \\
\text { T7. The enthalpy of this state is the same as that of } \\
\text { State } 4 .\end{array}$ \\
\hline State 2 & - & Saturated vapor at temperature $\mathrm{T} 2$. Note that $\mathrm{T} 2=\mathrm{Tl}$. \\
\hline State ISH & - & $\begin{array}{l}\text { Superheated vapor at Pressure PISH }=\sqrt{P 2 \times P 3 . ~ T h e ~} \\
\text { temperature varies with compressor efficiency. }\end{array}$ \\
\hline State ISAT & - & $\begin{array}{l}\text { Saturated vapor at pressure PISAT }=\text { PISH. Note that } \\
\text { the refrigerant mass flow rate is increased during the } \\
\text { saturation process. }\end{array}$ \\
\hline State 3 & - & $\begin{array}{l}\text { Superheated vapor at pressure P3. The temperature of } \\
\text { this state varies with compressor efficiency. }\end{array}$ \\
\hline State $3 A$ & - & $\begin{array}{l}\text { Saturated vapor at pressure } P 3 A=P 3 \text {. Note that the } \\
\text { refrigerant mass flow rate was increased during sat- } \\
\text { uration process. }\end{array}$ \\
\hline State 3B & - & $\begin{array}{l}\text { Saturated liquid at pressure } P 3 B=P 3 A \text {. Part of this } \\
\text { liquid is used as make up for accumulator tank } \frac{\|}{\pi} 2 \text {. }\end{array}$ \\
\hline State 30 & - & $\begin{array}{l}\text { Subcooled liquid at temperature T3C = TISAT. AII } \\
\text { properties except pressure are essentially the same } \\
\text { as State } 4 \text {. }\end{array}$ \\
\hline State 4 & - & Saturated liquid at temperature T4 = TISAT. \\
\hline
\end{tabular}


TABLE 3.1 (Cont'd)

Air Water Vapor:

\begin{tabular}{|c|c|c|}
\hline State 5 & - & $\begin{array}{l}\text { The properties at State } 5 \text { (i.e. T5 and RH5) are } \\
\text { process controlled variables which must be measured } \\
\text { for each application. Typical values to be used in } \\
\text { analysis are } T 5=205^{\circ} \mathrm{F} \text {, RH5 }=85 \% \text {. }\end{array}$ \\
\hline State 53 & - & $\begin{array}{l}\text { Intensive properties are the same as State } 5 \text {; flow } \\
\text { rate has been reduced to achieve energy balance. }\end{array}$ \\
\hline State 6 & - & $\begin{array}{l}\text { Saturated mixture of air and water vapor at temperature } \\
\text { T6. }\end{array}$ \\
\hline State 7 & - & $\begin{array}{l}\text { The properties of State } 7 \text { are determined by other system } \\
\text { variables affecting heat released in heat exchanger } C \text {. } \\
\text { Note that specific humidity at } 7 \text { is same as specific } \\
\text { humidity at } 6 \text {. }\end{array}$ \\
\hline State 15 & - & $\begin{array}{l}\text { Liquid water condensed from air water-vapor stream. } \\
\text { Temperature } T 15 \text { is approximately the same as T6. }\end{array}$ \\
\hline \multicolumn{3}{|c|}{ Steam Loop: } \\
\hline State 12 & - & $\begin{array}{l}\text { The properties at State } 12 \text { (i.e. } T 12 \text { and } \mathrm{P} 12 \text { ) are } \\
\text { process controlled variables which must be evaluated for } \\
\text { each application. Typical values to be used in analys is } \\
\text { are } T 12=302^{\circ} \mathrm{F}, \mathrm{P} 12=75 \text { PSIA, liquid. }\end{array}$ \\
\hline State 13 & $=$ & $\begin{array}{l}\text { The properties at state } 13 \text { (i.e. } \mathrm{T} 13 \text { and } \mathrm{P} 13 \text { ) are process } \\
\text { controlled variables. which must be evaluated for each } \\
\text { application. Typical values to be used in analys is are } \\
T 73=307^{\circ} \mathrm{F} \text {, P } 73=75 \text { PSIA, saturated vapor. }\end{array}$ \\
\hline
\end{tabular}

The analysis in this section is for the general case of an indirect drying process which has been fitted with a heat pump was te heat recovery system as shown in Figure 3.1. The nomencalture is given in Table 3.2. 
TABLE 3.2 NOMENCLATURE

\begin{tabular}{|c|c|c|c|}
\hline$C p$ & Specific heat & \multicolumn{2}{|c|}{ Subscripts } \\
\hline$F$ & $\begin{array}{l}\text { Fraction of air dis- } \\
\text { charged from dryer to } \\
\text { a tmosphere }\end{array}$ & $\begin{array}{l}1,2,3 A . . \\
\text { ISH, ISAT }\end{array}$ & $\begin{array}{l}\text { State point } \\
\text { locations }\end{array}$ \\
\hline $\mathrm{h}$ & Enthalpy & $(S)$ & Isentropic state \\
\hline $\mathrm{m}$ & Mass flow rate & A & Air \\
\hline$p$ & Pressure & $R 1, R 2, R 3$ & Refrigerant \\
\hline$s$ & Entropy & $P$ & Product (paper \\
\hline$T$ & Temperature & & \\
\hline$\emptyset$ & Relative humidity & $W$ & Water \\
\hline w & Specific humidity & $\begin{array}{l}F \\
g\end{array}$ & $\begin{array}{l}\text { Saturated Liquid. } \\
\text { Saturated vapor }\end{array}$ \\
\hline$\eta_{C}$ & Compressor efficiency & & \\
\hline
\end{tabular}

In analyzing the performance of an actual system certain conditions must be specified. Those conditions which are characteristic of the particular process being considered are designated as process controlled variables and are defined in Table 3.3. Other conditions termed system variables are not controlled directly by the process application but are determined as a part of the heat pump design procedure. These are defined in Table 3.4. 


\section{$\cdot 3.6$}

TABLE 3.3

\begin{tabular}{|c|c|c|}
\hline T5 & - & $\begin{array}{l}\text { The temperature of the air water-vapor mixture exhausted } \\
\text { from the dryer. }\end{array}$ \\
\hline 95 & - & $\begin{array}{l}\text { The relative humidity of the air water-vapor mixture exhausted } \\
\text { from the dryer. }\end{array}$ \\
\hline Mp & - & The mass flow rate of product through the dryer (Ib/HR) \\
\hline$M_{W 16}$ & - & The mass flow rate of water in the product entering dryer. \\
\hline$M_{W 17}$ & - & The mass flow rate of water in the product exiting dryer. \\
\hline $\mathrm{H} 12$ & - & Enthalpy of condensate exiting drum dryers. \\
\hline H13 & - & Enthalpy of steam provided to drum dryers. \\
\hline
\end{tabular}


TABLE 3.4

\begin{tabular}{|c|c|}
\hline$T$ & Design temperature of the refrigerant in the evaporator. \\
\hline T3A & Design temperature of the refrigerant in the condenser. \\
\hline T6 & $\begin{array}{l}\text { The temperature of the air water-vapor mixture down stream } \\
\text { of heat exchanger } E \text {. }\end{array}$ \\
\hline$F$ & $\begin{array}{l}\text { The fraction of the mass flow of the air water-vapor mixture } \\
\text { removed from system for an energy balance. }\end{array}$ \\
\hline PISH & The intermediate stage pressure defined as PISH $=\sqrt{\mathrm{P} 2 \times \mathrm{P3}}$. \\
\hline T4 & $\begin{array}{l}\text { The refrigerant temperature down stream of heat exchanger } C \\
(T 4=\text { TISAT). }\end{array}$ \\
\hline$T 7$ & $\begin{array}{l}\text { The temperature of the air water-vapor mixture down stream of } \\
\text { heat exchanger } C \text {. TT is calculated from an energy balance on } \\
\text { heat exchanger } C \text {. }\end{array}$ \\
\hline$\pi_{c}$ & The compressor efficiency. \\
\hline
\end{tabular}

\section{Refrigeration Cycle}

By specifying the evaporator and condenser temperatures the corresponding pressures can be found from saturation data for the refrigerant. Figure 3.2 shows the thermodynamic cycle for the refrigerant in the heat pump. The ideal (isentropic) and actual compressor processes are shown in Figure 3.2.

State Point 2 is considered to be a saturated vapor at temperature T'L. The properties at State 2 are found to be:

$$
\begin{aligned}
& \mathrm{h} 2=\mathrm{hg} \odot \mathrm{T} 2 \\
& \mathrm{S2}=\mathrm{Sg} \odot \mathrm{T2} \\
& \mathrm{P2}=\mathrm{Pg} \odot \mathrm{T2}
\end{aligned}
$$




\section{8}

FIGURE 3.2

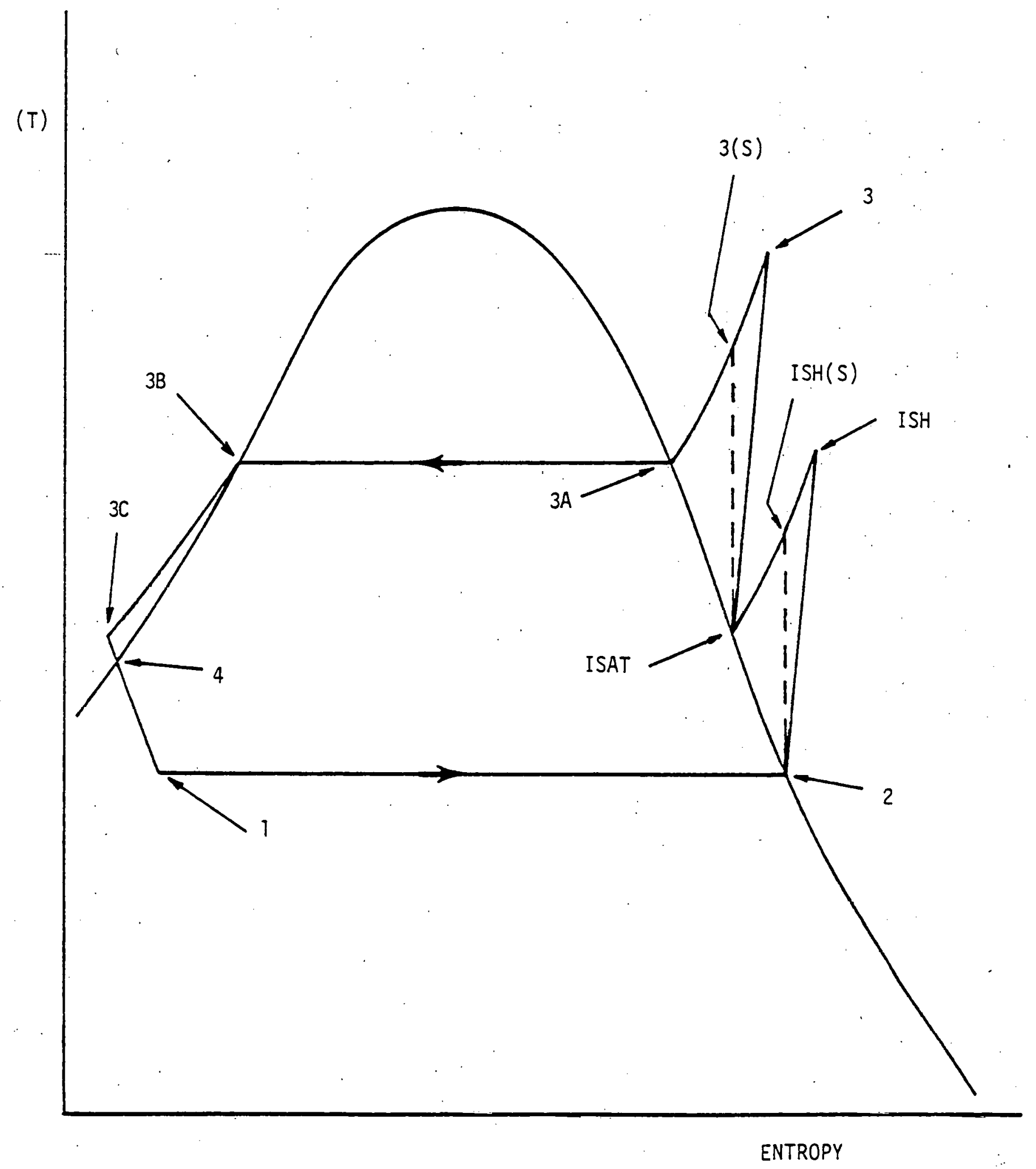


The pressure at State Point $3 A$ is found to be:

$P 3 A=P g \subseteq T 3 A$

For an isentropic compression state ISH(S) is determined:

PISH $==$ PISAT $=\sqrt{P 2 \times P 3 A}$

$\operatorname{SISH}(S)=S 2$

$h I S H(S)=h$ \& PISH, SISH(S)

The definition of compressor efficiency is:

$n_{c}=\frac{h I S H(S)-h 2}{h I S H-h 2}$

which can be rearranged to give the actual compressor discharge enthalpy for the first stage compressor

$$
h I S H=\frac{h I S H(S)-h 2}{n_{C}}+h 2
$$

The saturation process in both accumulator tanks is assumed to be isobaric. The properties at State ISAT are fixed as:

PISAT = PISH

TISAT $^{\circ}=\mathrm{Tg}$ O PISAT

hISAT $=$ hg $Q$ PISAT

SISAT $=S g$ P PISAT

For an isentropic compression. State $3(S)$ is determined:

P3 $=P 3 A$

$S 3(S)=$ SISAT

$h 3(S)=h @ P 3 A, S 3(S)$

The compressor efficiency is given by:

$n_{c}=\frac{h 3(S)-h I S A T}{h 3-h I S A T}$

The actual discharge enthalpy of the second stage compressor is:

h3 $\frac{h 3(S)-h I S A T}{n_{c}}+h$ ISAT 
The following properties of State $3 A$ are fixed by the saturation temperature T3A:

$$
\begin{aligned}
& P 3 A=P g @ T 3 A \\
& h 3 A=h g \subseteq T 3 A
\end{aligned}
$$

State $3 B$ is a saturated liquid at a temperature T3B. The following properties are determined at State $3 B$.

$T 3 B=T 3 A$

P3B $=P 3 A$

$h 3 B=h f$ O T $3 B$

State $3 C$ is a subcooled liquid at a pressure $P 3 C=P 3 B$ and a temperature $T 3 C=$ TISAT.

The enthalpy at State $3 C$ is the same as State 4 namely:

$$
h 3 C=h f @ T 4
$$

State 1 is a saturated mixture of liquid and vapor. The temperature $\mathrm{Tr}$ is given by:

$$
T 1=T 2
$$

The expansion process is assumed adiabatic so that:

$$
h 7=h 4=h 3 C
$$

The analysis of the air loop begins at State Point 5 where the properties T5 and 95 are known. The partial pressure of the water vapor of State 5 can be calculated by:

$$
\text { PW5 }=\emptyset \times P_{5} 5
$$

where $\mathrm{Pg} 5$ is the saturation pressure for the temperature, T5. The specific humidity at this state is:

$$
\text { W5 }=\frac{.622 \text { PW5 }}{\text { P5 }- \text { PW5 }}
$$

At State 6 the water vapor is saturated so that:

$$
\text { W6 }=\frac{.622 P g 6}{P 6-P g 6}
$$


The rate of water removal from the product is:

$M_{W}=M_{W 16}-M_{W 17}$

The mass flow rate of air at State 5 is:

MA5 $=M_{W} /(W 5-W 6+F \times W 6-F \times W A)$

The mass flow rate of water at State 5 is:

$M_{W 5}=W 5 \times M_{A 5}$

The following equations are determined from mass balances:

$M_{W 53}=(1-F) \times M_{W 5}$

$M_{A 53}=(1-F) \times M_{A 5}$

$M_{W 6}=M_{W 5}-M_{W}$

$M_{W 15}=M_{W 53}-M_{W 6}$

An energy balance on heat exchanger $E$ gives:

$M_{R 1}=\left(M_{W 53} \times h_{W 53}-M_{W 6} \times h_{W 6}+M_{A 53} \times C P A \times(T 5-T 6)\right.$

- $\left.M_{W 15} \times h_{W 15}\right) /(h 2-h 1)$

An energy balance on the first stage of compression gives the compressor work as:

WDOT1 $=M_{R 1}$ (hISH - h2)

An energy balance on accumulator Tank No. 1 gives:

$M_{R 2}=M_{R 1} \times(h I S H-h 4) /(h I S A T-h 4)$

An energy balance on the second stage compressor gives:

WDOT2 $=M_{R 2} \times(h 3-h I S A T)$

The total compressor work is:

WTOT $=$ WDOTI + WDOT2 
Making an energy balance on the entire system gives:

$$
\begin{aligned}
\text { WTOT1 } & =-\left(M_{A 5} \times C_{P A} \times F \times(T A-T 5)+M_{W 16} \times h_{W 16}-M_{W 17}\right. \\
& \times h_{W 17}+M_{P} \times C_{P P} \times(T 16-T 17)-M_{W 5} \times h_{W 5} \times F \\
& \left.-M_{W 15} \times h_{W 15}\right)
\end{aligned}
$$

Equation [16] is used to solve for WTOTI. For an energy balance on the system the value of the variable $F$ must be such that WTOT $=$ WTOTI. An energy balance on accumulator Tank No. 2 gives:

$$
M_{R 3}=M_{R 2} \times(h 3-h 3 B) /(H 3 A-h 3 B)
$$

The mass flow rate $M_{R 3}$ only flows through heat exchanger ABOT.

Now that all the needed state point properties and mass filow rates are specified directly or expressed in terms of known properties a solution procedure can be established.

\section{Solution Procedure}

a Specify a value of $\mathrm{T2}$

b Solve Equation [1] for h2

c Solve Equation [2] for $\$ 2$

d. Solve Equation [3] for P2

e Specify a value for T3A

$f \quad$ Solve Equation [4] for P3A

g Solve Equation [5] for PISH

h Solve Equation [6] for SISH(S)

i Solve Equation [7] for hISH(S)

$j$ Solve Equation [9] for hISH

$k$ Solve Equation [10] for PISAT 
1 Solve Equation [11] for TISAT

$m$ Solve Equation [12] for hISAT

$\mathrm{n}$ Solve Equation [13] for SISAT

- Solve Equation [14] for P3

$p$ Solve Equation [15] for $S 3(S)$

q Solve Equation [16] for $h 3(S)$

$r \quad$ Solve Equation [18] for $h 3$

s Solve Equation [19] for P3A

$t$ Solve Equation [20] for $h 3 A$

u Solve Equation [21] for T3B

$v \quad$ Solve Equation [22] for P3B

$w \quad$ Solve Equation [23] for $h 3 B$

$x \quad$ Solve Equation [24] for $h 4$

$y \quad$ Solve Equation [25] for $T I$

$z \quad$ Solve Equation [26] for $h l$

aa Solve Equation [27] for PWA

bb Solve Equation [28] for W5

cc Solve Equation [29] for W6

dd Solve Equation [30] for $M_{W}$

ee Assume a value of $F$

ff Solve Equation [31] for $M_{A 5}$

gg Solve Equation [32] for $M_{W 5}$

hh Solve Equation [33] for $M_{W 53}$

ii Solve Equation [34] for $M_{A 53}$

jj Solve Equation [35] for $M_{W 6}$

kk Solve Equation [36] for MW75 
11 Solve Equation [37] for $M_{R T}$

mom Solve Equation [38] for WDOT1

$\mathrm{nn}$ Solve Equation [39] for $M_{R 2}$

oo Solve Equation [40] for WDOT2

pp Solve Equation [41] for WTOT

qq Solve Equation [42] for WTOT1

If WTOTI $\neq$ WTOT, then an energy balance has not been established.

By adjusting the value chosen for $F$ and repeating Steps ee through qq an energy balance can be achieved such that WTOTI = WTOT.

$r$ Solve Equation [43] for $M_{R 3}$

For the Heat Exchangers

Heat transfer in heat exchanger ABOT:

QABOT $=M_{R 3} \times(h 3 A-h 3 B)$

Heat transfer in heat exchanger $C$ :

QC $=M_{R 2} \times(h 3 B-h 3 C)$

Heat transfer in heat exchanger $E$ :

$Q E=M_{R 1} \times(h 2-h 1)$

The procedure just outlined has been modified and written in Fortran language for computer application.

The computer analysis actually involves the use of two computer programs to be used in sequence. The first program entitled HPF2 gives the system performance for various combinations of system variables. The information provided by this program is a basic overview of performance and economic attractiveness of a system operating under the specified conditions. 
3.15

By comparing this information for each combination of system variables the designer, can then choose the conditions which yeild optimum performance and feasibility. The second program entitled HPOP2 gives more detailed information about the system performance at the conditions chosen. This program also gives component sizing information necessary for design. The two computer programs are shown in the following pages. 
COMMON/PAUL/S $(35,48)$

COMMON/TOM/H $(35,48)$

COMMON/GLEN/V $(35,48)$

OPEN (UNIT $=24$, TYPE $=$ 'OLD', NAME='VDAT. DAT', READONLY)

DPEN (UNI T =22, TYPE $=$ 'QLD', NAME ='SDAT. DAT', READONLY)

OPEN (UNIT $=20$, TYPE $=$ 'OLD', NAME $=$ 'HDAT. DAT', READONLY)

DO 87 I $X=1,8$

$N=(I X-1) * 6$

READ (24, 80)

$\operatorname{READ}(22,80)$

READ (20,80)

80 FORMAT ( 1 HO)

DO 90 IY $=1,35$

$\operatorname{READ}(24,81) V(I Y, N+1), V(I Y, N+2), V(I Y, N+3), V(I Y, N+4)$,

$\& V(I Y, N+5), V(I Y, N+6)$

READ $(22,81) S(I Y, N+1), S(I Y, N+2), S(I Y, N+3), S(I Y, N+4)$,

$\& S(I Y, N+5), S(I Y, N+6)$

READ $(20,81) H(I Y, N+1), H(I Y, N+2), H(I Y, N+3), H(I Y, N+4)$,

\& $H(I Y, N+5), H(I Y, N+6)$

81 FORMAT (9X, 6F 10.3)

90 CONTINUE

87 CONTINUE

$T G M(P)=.9100 E+02+.4268 E+01 * P-.5102 E-01 * P * 2 .+.3531 E-03 * P * 3$.

\&-. $1287 E-05 * P * 4$. +. 2267E-08*P*5. - $1336 E-11 * P * 6 .-.4030 E-15 * P * * 7$.

$P G M(T)=-.1824 E+02+.2724 * T-.6738 E-03 * T * 22$.

\&-. 2364E-05*T*3. +. 2102E-07*T*4.

$\&+1198 \mathrm{E}-09 * \mathrm{~T} * 5 .-.4317 \mathrm{E}-12 * \mathrm{~T} * * 6$.

$\&+.4933 E-15 * T * * 7$.

HSVM $(T)=.498 E+03+.4096 * T-.611 E-03 * T * 2$.

\&-. $115 E-05 * T * 3 .+.211 E-07 * T * 4$.

\&-. $1835 E-09 * T * * 5 .+.6793 E-12 * T * * 6$.

\& $-.8694 \mathrm{E}-15 * T * * 7$.

HSLM $(T)=-.1047 E+03+.1675 E+01 * T-.1107 E-02 * T * 2$.

$\&-.3109 E-04 * T * * 3 .+.814 E-07 * T * * 4$.

\&4. 4744E-09*T*5. $-.226 E-11 * T * 6$.

$\&+.2589 E-14 * T * * 7$.

$\operatorname{SSUM}(T)=.9598+.8032 E-03 * T-.1172 E-04 * T * 2$.

\&+. 1973E-07*T**3. +. 9589E-10*T**4.

\&-. $4041 E-12 * T * * 5 .+.4968 E-15 * T * * 6$.

\&-. $1865 E-18 * T * * 7$.

THESE CALCULATIONS FOR HIGH LOW TEMP IN HEAT PUMP

ST HELENS DATA

DATA T1,H1, ETAC/180.0,146.6,0.90/

DATA T2, H2/180. 0,552. O/

DATA TISH, HISH/305. 0,600. O/

DATA TISAT, HISAT/250.0,560.4/

DATA T3, H3/370. 0,640. O/

DATA T3A, H3A/325. $0,565.0 /$

DATA T3B, H3B/325. 0, 212.7/

DATA T4, H4/250.0,146.6/

DATA T5, T6, T12/205. 0, 190.0,302.0/

DATA T15, T16, T17, TA/190.0,150.0,230.0,100.0/

DATA H12, H13/272. 8, 1182. 4/

DATA FP, FW16, FW17/740. 8, 606. 0, 47.3/

DATA RH5, WA/O. 85, 0.04/

DATA UATOP, UABOT, UC, UE/5. 0, 1000.0,5.0,5.0/

DATA SP, CPP, DE, ICF/1. 54,0.45,0.75,2.0/

DATA HOUR, DPAIR, DPAIR6/8000. 0,0.5,0.5/

DATA HPCOM, CELEC, CFUEL, CSTEAM/110.0,0.00001172, 0.000003,0.000004/

IF ITYPE $=2$ WE HAVE A STEAM TURB INE

REAL ICF 
I TYPE $=1$

$C A S E=2$

DO $2000 \quad K K=315,330,5$

$T 3 A=K K$

DO $2000 \quad M M=191,196,1$

$\mathrm{T} 6=\mathrm{MM}$

DO $2000 \quad N N=165,190,5$

$T 1=N N$

STATE $3 A$

$P 3 A=P G M(T 3 A)$

$H 3 A=H S V M(T 3 A)$

STATE 3B

$T 3 B=T 3 A$

$P 3 B=P 3 A$

$H 3 B=H S L M(T 3 B)$

STATE 4

$T 4=T I G A T$

$P 4=P 3 B$

$H 4=H S L M(T 4)$

2

$c$

c

STATE 1

$P 1=P G M(T 1)$

$H 1=H S L M(T 4)$

c.

: STATE 2

3

$T 2=T 1$

$P 2=P 1$

$H 2=H S U M(T 2)$

S2=SSUM (T2)

C

STATE ISH

S. ISH $=52$

PISH $=(P 2 * P 3 A) * 0.5$

$T$ ISH $=S H M S P(S I S H, P I S H)$

$H I S H P=H M S H(T I S H, P I S H, 2)$

$H I S H=(H I S H P-H 2) / E T A C+H 2$

$\underset{5}{c}$

STATE ISAT

PISAT $=P I S H$

TISAT $=$ TGM (P ISAT)

HISAT $=H S V M(T$ ISAT $)$

SISAT $=S S V M(T I S A T)$

C

C STATE 3

C

$P 3=P G M(T 3 A)$ 
S3P $=5 I S A T$

T3=SHMSP (S3P, P3)

$H 3 P=H M S H(T 3, P 3,2)$

$H 3=(H 3 P-H I S A T) / E T A C+H I S A T$

IF (ITYPE. EQ. 1) WRITE (6, 20)

20 FORMAT(1H, 30X, 'ELECTR I C M OT DR'////1)

IF (ITYPE. EQ. 2) WRITE $(6,21)$

21 FORMAT (1H, 30X, 'S T E A M TUR B I N E'/////)

2001 FORMAT $(1 H$, 'THESE CALCULATIONS FOR RH5=',F7. 2,' AND T5=',F7. 2/)

$\mathrm{c}$

C

$$
D F=.01
$$

PW5=RH5*PG(T5)

$W 5=.622 * P W 5 /(14.7-P W 5)$

$W 6=.622 * P G(T 6) /(14.7-P G(T 6))$

$P G G=P G(T G)$

'666 FORMAT (1H, 4E16.6)

$F W=F W 16-F W 17$

C

C

$F=0.0$

$1 \quad F=F+D F$

¿

IF (CASE. EQ. 1) $F=.02$ 
$F A 5=F W /(W 5-W 6+F * W 6-F * W A)$

$F W 5=W 5 * F A 5$

$F W 53=(1 . O-F) * F W 5$

$F A 53=(1, O-F) * F A 5$

$F W 6=F W 5-F W$

FW15=FW53-FW6

$H G=H T P(T 6, P G(T 6))$

H15 $=$ T 15-32. $O$

H53 $=H T P(T 5, P W 5)$

FR $1=(F W 53 * H 53-F W 6 * H 6+F A 53 * .24 *(T 5-T 6)-F W 15 * H 15) /(H 2-H 1)$

$H 16=T 16-32$.

$H 17=T 17-32$.

WDOT 1=FR I * (HISH-H2)/2545. 0

$F R 2=F R 1 *(H I S H-H 4) /(H I S A T-H 4)$

WDOT2=FR2* (H3-HISAT ) /2545. O

WTOT =WDOT 1 +WDOT2

E

C

IF (CASE. NE. 1) GO TO 675

$\Xi$

$P W A=W A * 14.7 /(.622+W A)$

$F W A=W A * F A 5 * F$

$H A=H T P(T A, P W A)$

FSEXT $=$ (2545. O*WTOT+FA5*. 24*F*(TA-T5)+FW16*(H16)-FW1 7*H17+FP*CPP*

$1(T 16-T 17)-F W 5 * H 53 * F-F W 15 * H 15+F W A * H A$

$/(\mathrm{H} 13-\mathrm{H} 12)$

E

$\mathrm{C}$

GO TO 3

5

C

675 CONTINUE

WTOT $1=-(F A 5 * .24 * F *(T A-T 5)+F W 16 * H 16-F W 17 * H 17+F P * C P P *(T 16-T 17)-F W 5 *$ $1 H 53 * F-F W 15 * H 15) / 2545$.

$c$

c

IF (WTOT1. GE. WTOT) GO TO 2

C

C

C

c

C

GOTO I

IF (ABS (WTOT1-WTOT). LE. O. OO1*WTOT) GO TO 5

$F=F-D F$

$\mathrm{DF}=\mathrm{DF} / 10.0$

GO TO 1

C

5 CONTINUE

FSEXT $=0.0$

3 CONTINUE

DTE $=(T G(P W 5)+T 15) * 5-T 1$

$Q E=F R 1 *(H 2-H 1)$

$A E=Q E /(U E * D T E)$

COETE $=A E * 3$

$V E=A E / 2000$.

$F R A=F A 53 /(F W 6+F A 53)$

$X 7=(\{1 .-F R A) / 18) /.((1 .-F R A) / 18 .+F R A / 28.93)$

$P W 7=X 7 * 14.7$

300 FORMAT (1H, 'DATA FOR HEAT EXCHANGER E') 
301 FORMAT(1H,'DT=',F10.1,' $A=\prime, F 10.1, ' C O S T=', F 10.1, ' Q=\prime, E 13.4$, 1 ' VOLUME $=\prime, F 10.1$ )

DTATOP $=(T 3+T 3 A) * 5-T 12$

QATOP =FR2* $(H 3-H 3 A)$

AATOP $=$ QATOP / (UATOP $* D T A T O P$ )

CATOP $=$ AATOP $* .3$

VATOP $=$ AATOP $/ 2000.0$

DTABOT $=T 3 A-T 12$

QABOT $=F R 2 *(H 3 A-H 3 B)$

$A A B O T=Q A B O T /(U A B O T * D T A B O T)$

$C A B O T=A A B O T * 10.0$

$\checkmark A B O T=A A B D T / 20.0$

$Q C=F R 2 *(H 3 B-H 4)$

$T 7=T 6$

$\mathrm{DT} 7=5.0$

$4 \quad T 7=T 7+D T 7$

$Q C 1=F A 53 * .24 *(T 7-T 6)+F W 6 *(H T P(T 7, P W 7)-H 6)$

c

$\operatorname{IF}(Q C 1 . G E . Q C)$ GO TO 6

c

GO TO 4

C

6

c

C

IF $(A B S(Q C 1-Q C)$. LE. $0.001 * Q C)$ GO TO 8

$T 7=T 7-D T 7$

$D T 7=D T 7 / 10.0$

GO TO 4

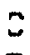

8 CONTINUE

$H 7=H T P(T 7, P W 7)$

$D T C=((T 3 A+T 4)-(T 6+T 7)) * .5$

$A C=Q C /(U C * D T C)$

$\operatorname{COSTC}=A C * .3$

$V C=A C / 2000.0$

305 FORMAT ( $1 \mathrm{H}$, 'DATA FOR HEAT EXCHANGER $\mathrm{C}$ ')

302 FORMAT (1H, 'DT $=\prime, F 10.1, ' A=\prime, F 10.1, ' C O S T=\prime, F 10.1$,

1' $Q=\prime, E 13.4$,' $V O L U M E=\prime, F 10.1, ' T 7=\prime, F 10.1$ )

304 FORMAT ( $1 \mathrm{H}$, 'DATA FOR BOTTOM OF HEAT EXCHANGER A')

303 FORMAT ( $1 H$, 'DATA FOR TOP OF HEAT EXCHANGER $A^{\prime}$ ')

CCOMP $=$ HP COM*WTOT .

$C P M=W T O T * 20$.

$c$

IF (ITYPE. EQ. 2) CPM=WTOT $* 90$.

$C T O T=C A T O P+C A B O T+C O S T C+C O S T E+C C O M P+C P M$

$T=T 6+460$.

$R H O G=P G(T 6) * 144.0 /(1545.0 * T / 18.0)+(14.7-P G(T 6)) * 144.0 /(53.3 * T)$ HPFAN $=$ (FA53+FW6) *144. /(RHO6*778. *254.5)

FSTEAM $=(Q A T O P+Q A B O T) /(H 13-H 12)$

DHBOIL $=H 13-H 12$

QBOIL $=$ DHBOIL $*$ FSTEAM/BE

QPM $=W T O T * 2545 . /(.3 * .95)$

IF (ITYPE. EQ. 1) GO TO 11

PMST $=$ WTOT*2545. /(385, *. 65)

$c$

$Q P M=P M S T * D H B O I L / B E$

GO TO 12 


\section{CONTINUE}

PMST $=0$

12 CONTINUE

HPFANS $=$ HPFAN $* D P A I R G$

$E R=Q B D I L+Q C-Q P M-H P F A N B * 2545$.

3000 FORMAT $(1 \mathrm{H}$, 'COST OF COMPRESSOR=', F10.1,10X,'COST OF PRIME MOVER=',

1 F1O. 1)

$E R 2=S P * F P * D H B O I L / B E-Q P M-H P F A N G * 2545$

FASTHE $=F W /(W 5-W A)$

FW5THE =W5*FA5THE

FWATHE $=F A S T H E * W A$

QTHE $=F A 5 T H E *, 24 *(T 5-T A)+F W 5 T H E * H 53-F W A T H E * H A$

$\&+F P * C P P *(T 17-T 16)+F W 17 * H 17-F W 16 * H 16$

$S P T=Q T H E /(D H B Q I L \# F P)$

ER3 $=Q T H E / B E-Q P M-H P F A N G * 2545$.

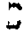

IF (CASE. NE. 1 ) GO TO 766

ב

$B B B=F S E X T *(H 13-H 12) / B E$

$E R=E R+B B B$

$E R 2=E R 2+B B B$

$E R 3=E R 3+B B B$

766 CONTINUE

400 'FORMAT (1H, 'SOME SUMMARY FIGURES'/1H, 'ENERGY RECOVERY RATE=',

1 E13. 4, 10X, 'REFRIGERANT FLOW=', E13. 4/1H , 'COMPRESSOR HORSEPDWER=', 2E13. 4/1H , 'ENERGY RECDVERY BASED ON. ', F5. 2 ,' LB 0 3F STEAM PER PQUND OF PAPER', E13. 4/1H, 'ENERGY RECOVERY BASED ON TH 4EDRETICAL REQUIREMENT OF',F5. 2 ,' LB QF STEAM PER POUND OF PAPER $=$ ', SE13. 4)

C

IF (ITYPE. EQ. 2) FCOST=WTOT*2. 545*350. *24. *CSTEAIM/1000.

こ

IF(ITYPE. EQ. 1) FCOST=WTOT*. 7457*24. *350. *CELEC/(1000.*. 95)

$\mathrm{C}$

SCOST $=S P * F P * 350 . * 24 . *$ CSTEAM $/ 1000$.

SCOST $2=S P * F P * D H B O I L * 24 . * 350 . * C F U E L /(B E * 1$. OEG)

5000 FORMAT 1 1H, 'ANHUAL COST TO RUN PRIME MOUER=?, F10.1,' ANNUAL COST 1 IOF STEAM BASED ON CSTEAM=', F10.1/1H, 'ANNUAL COST OF STEAM BASED O IN BOILER FUEL REQUIRED AT COST OF CFUEL=', F1O. 1)

4123 FORMAT $\left(1 H,{ }^{\prime} F A 5=\prime, F 10.1\right.$,' $F W 5=\prime, F 10.1, '$ FAG =',F10.1,' FWG $=$ ',

1 F10.1)

WRITE(6, 401) F, CTOT

401 FORMAT(IH, 'FRACTION OF AIR THROWN AWAY=',F6. 4/

$31 \mathrm{H}$, 'TOTAL COST OF MAJOR COMPONENTS=', F10.1)

402 FORMAT ( $1 \mathrm{H}$, 'FAN HORSEPOWER PER PSI DROP=', F10. 4)

1000 FORMAT (1H, 'STEAM FLOW=', E13. 4, 10X,'BOILER HEAT INPUT WITHOUT HEAT

1 PUMP =', E13. 4/1H , 'HEAT RATE TO PRIME MOVER=', E13. 4, 10X,

2 'LB OF STEAM/LB PAPER WITHOUT HEAT PUMP=', E13. 4)

767 FORMAT (1H , 'EXTRA STEAM=', E13. 3,' EXTRA ENERGY=', E13. 3,' H6=',

$1 E 13.3, ' H 7=\prime, E 13.3, ' H 53=\prime, E 13.3)$

$\mathrm{C}=4.0 / 3.14$

$T=T 5+460.0$

$R H O 5=(14.7-P W 5) * 144.0 /(53.3 * T)+P W 5 * 144.0 /(85.8 * T)$

$F .5=F A 5+F W 5$

$V 5=30.0 * 3600.0$

$A S=F 5 /(R H O 5 * V 5)$

$D S=S Q R T(C * A 5)$

$F G=F A 5 *(1.0-F)+F W G$

$V G=30.0 * 3600.0$ 
$A G=F G /(R H O G * V G)$

$D G=S Q R T(C * A G)$

$\mathrm{RHOT}=\mathrm{RHD} *(T 6+460.0) /(T 7+460.0)$

$V 7=30.0 * 3600.0$

$A 7=F 6 /(R H Q 7 * V 7)$

$D 7=\operatorname{SQRT}(\mathrm{C} * A 7)$

RHO2 $=1.0 \% .9594$

$V 2=18.0 * 3600.0$

$A E=F R 1 /(R H O 2 * V 2)$

$D 2=S Q R T(C * A 2)$

$\mathrm{RHO3}=1.0 / .283$

$V 3=18.0 * 3600.0$

$A 3=F R 2 /(R H 03 * V 3)$

$D 3=S Q R T(C * A 3)$

RHO4=1. $0 \% .0114$

$V 4=10.0 * 3600.0$

A4=FRE / (RHO4\#V4)

$D 4=S Q R T(C * A 4)$

$\mathrm{RHO1}=1.0 / .2935$

$V_{1}=15.0 * 3600.0$

$A 1=F R 1 /(R H O 1 * V 1)$

$D 1=S Q R T(C * A 1)$

C

IF(ITYPE. EQ. 1) GO TO 25

VSTM $=30.0 * 3600.0$

RHOSTM $=1.0 / 1.032$

ASTM $=P M S T /($ VSTM $*$ RHOSTM)

$D Q=S Q R T(C * A S T M)$

25 CONTINUE

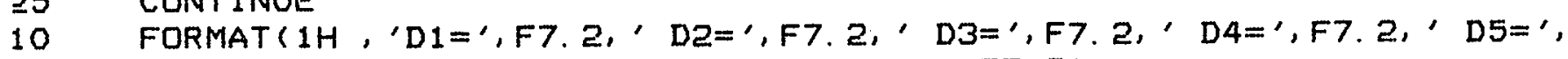

1 F7. 2,' $\left.D 6={ }^{\prime}, F 7.2, ' D 7=\prime, F 7.2, ' D 8=\prime, F 7.2\right)$

RHO1 $2=58.8$

RHO13=. 1

$V 12=30.0 * 3600.0$

$V 13=10.0 * 3600.0$

A12=FSTEAM/ (RHO12*V12)

$A 13=F 5 T E A M /(R H Q 13 * V 13)$

D12=SQRT $(C * A 12)$

D13=SQRT $(C * A 13)$

DPSTM $=288$. 0

FANHP $=F 5 * D P A I R * 144 /(R H 05 * 778.0 * 2545.0)$

PUMPHP $=$ FSTEAM $*$ DPSTM $/(R H 012 * 778.0 * 2545.0)$

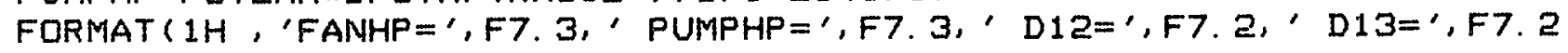
1)

EPRE $=S P * F P * H O U R * D H B O I L / B E$

EFUT $=($ QPM $+($ FANHP +HPFANG $) * 2545 / 0.3) * H O U R$

ESAVE $=E P R E-E F U T$

WRITE (6, 975) EPRE, EFUT, ESAVE

QPMA $=W T O T * 2545$

IF (ITYPE. EQ. 1) COSTF $=$ (QPMA+(HPFANG+FANHP )*2545)*HDUR*CELEC

IF (ITYPE. EQ. 2) COSTF = (QPM*CFUEL+(HPFANG+FANHP)*2545*CELEC )*HOUR COSTP=EPRE *CFUEL

FIRSTC $=$ CTOT*ICF

ROI $=($ COSTP - COSTF-FIRSTC $/ 10) / F I R S T C$

$C Q P=(Q A T Q P+Q A B Q T+Q C) /(W T Q T * 2545)$ 
$P P=F I R S T C /(C O S T P-C O S T F)$

WRITE (6, 309) ROI, COP, PP, T?

WR I TE (6, 308) T1, TISH, T4, T3

WRITE (6, 307) T3A, T5, T6, RH5

WRITE (6, 306) COSTP, COSTF, FIRSTC

FORMAT ( $1 \mathrm{H},{ }^{\prime}$ COSTP =', E12. 5, 3X, 'COSTF=', E12. 5, 3X, 'FIRSTC=', E12. 5)

309

FORMAT ( $\left.1 H,{ }^{\prime} R O I=', F 7.3,3 X,{ }^{\prime} C O P={ }^{\prime}, F 7.3,3 X, ' P P=\prime, F 7.3,3 X, ' T 7=\prime, F 7.3\right)$

308

FORMAT ( $1 H,{ }^{\prime}$ 'T1 1 ', F8. $1,3 X,{ }^{\prime}$ TISH=', F6. $\left.2,3 X, ' T 4=\prime, F 7.3,3 X, ' T 3=\prime, F 7.3\right)$

307

FORMAT ( $1 H,{ }^{\prime}$ T $3 A=\prime$, F7. 3, 3X, 'T5=', F8. 3, 3X, 'T6=', F7. 3, 3X, 'RH5=', F7. 3)

975

FORMAT ( 1 HO, 'PRESENT BTU $=$ ', E12. $5,3 X$, 'FUTURE

2000

1 BTU =', E12. 5, 3X, 'SAVED BTU=', E12. 5/'

CONTINUE

STOP

END

5

a

$\mathrm{C}$

FUNCTION $\operatorname{HTP}(T 1, P 1)$

$T=255.38+(T 1) / 1.8$

$P=P 1 / 14.6959$

$B 1=(2641.62 * 10 . *(80870.0 /(T * T))) / T$

$B O=1.89-B 1$

$B 2=82.546$

$B 3=162460.0 / T$

$B 4=.21828 * T$

$B 5=126970.0 / T$

$F O=1.89-B 1 *(372420.0 /(T * T)+2.0)$

$B G=B O * B 3-2 . O * F O *(B 2-B 3)$

$B 7=2.0 * F O *(B 4-B 5)-B O * B 5$

$B B=.4342944 * A L D G(T)$

$F=775.596+(.63296+.0001624 \quad * T) * T+47.3635 * B 8$

$B 9=B O * P * P /(2, O * T * T)$

$H T P=F+.043557 *(F O * P+B 9 *(B O *(B 2-B 3+2 . O * B 7 * B 9)-B 6))$

RETURN

END

FUINCTION TG(P)

IF (P. GT. 11.526) GO TO 1

$T G=38.795885+102.3232 * P-53.76781 * P * 2 .+17.63585 * P * 3 .-3.338111 *$

* $P * 4 .+3567123 * P * 5 .-1.994707 E-2 * P * 6$. $+4.5293 E-4 * P * * 7$.

GO TO 2

$1 T G=147.4684+5.665215 * P-.1045811 * P * 2 .+1.21192 E-3 * P * * 3$.

* $\quad-7.2 E-6 * P * 4 .+2 . O E-8 * P * 5$.

2 CONTINUE

RETURN

END

C

$\mathrm{C}$

FUNCTION PG(T)

DIMENSION $F(B)$

DATA $F / m 741,9242,-29.721,-11.55286,-8685635$,

\& . 1094098, . 439993, .2520658, .05218684/

TFAR $=T$

$A=0.0$

$\mathrm{TC}=5.19 . *$ (TFAR -32.$)$

$T A U=1000.1(T C+273.15)$ 
PCRIT $=220.88 * 14.5$

TCRIT $=374.136$

DO $20 \quad J=1,8$

$A=A+F(J) *(.65-.01 * T C) *(\nu-1)$

20 CONTINUE

$B=T A U *(1 . / 10 . *$ 5. $) *(T C R I T-T C)$

$C=A * B$

10 CONTINUE

$P G=P C R I T * E X P(C)$

RETURN

END

FUNCTION SHMSP (SI,PI)

DATA P/14. 7, 15.2, 18.7, 20., 23. , 28. 1, 30., 34. 1, 40.,41.1,

849 . 1, 50., 58. 4,60.,69.,70.,80.,81.,90.,95.,

$\$ 100,110,120,127,130,140,147,150,160 .$,

$8169,170,180,190,194 ., 200,210,220,221,, 230 .$,

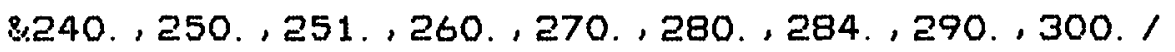

DATA T/150, , 160.,170., 180, 190.,200, 210.,221, 230.,

8240 . , 250. , 253. , 260. , 270. , 280., 281., 290.,

\$300, , 302., 310., 320., 330., 334., 340, 350.,

360, 370., 380, , 390., 400, 410, 420.,

$8430 ., 440,450.1$

DIMENSION T(35), P (48), ST (35)

COMMON/PAUL/ $S(35,48)$

DO $10 \quad J=1,48$

IF (PI. LE. $P(1+1))$ GOTO 15

10 CONTINUE

$15 \mathrm{DO} 20 \quad \mathrm{I}=1,35$

$Q 1=(P(J)-P(J+1)) /(P(J)-P I)$

$A=S(I, J)$

$B=S(I, J+1)$

$S T(I)=A-((A-B) / Q 1)$

20 CONTINUE

DO $25 \quad J=1,35$

IF(SI. LE. ST $(J+1)$, GOTO 30

25 CONTINUE

$30 \operatorname{QE}=(\operatorname{ST}(J)-\operatorname{ST}(J+1)) /(\operatorname{ET}(J)-E I)$

$A=T(\sqrt{)})$

$B=T(v+1)$

$S H M S P=A-((A-B) / Q 2)$

RETURN

END

FUNCTION HMSH(TI,PI, IF)

DIMENSION $T(35), P(48)$

COMMON/TOM/ $H(35,48)$ 


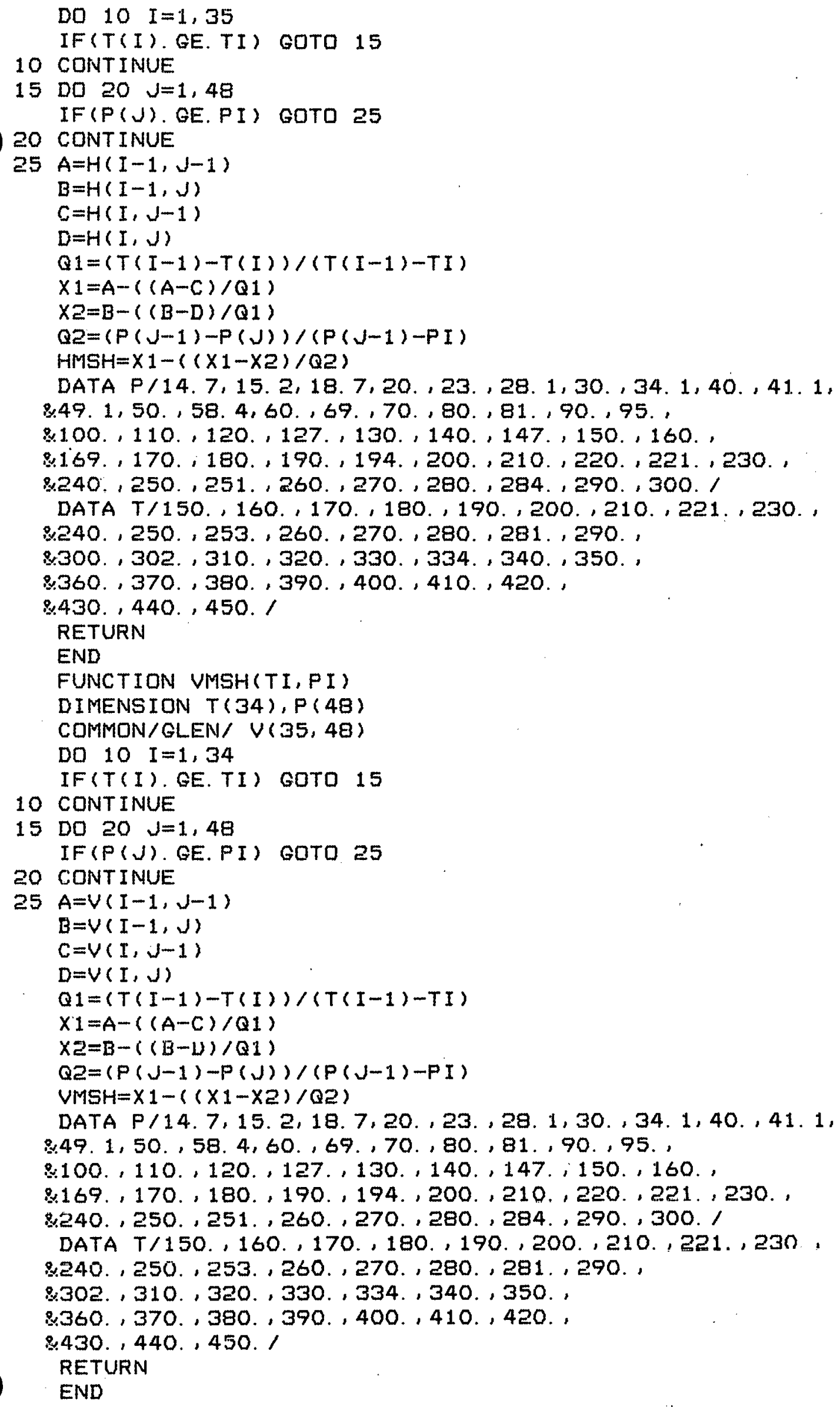


COMMON/PAUL/S(35,48)

COMMON/TOM/H $(35,48)$

COMMON/GLEN/VZ $(35,48)$

OPEN (UNIT=24, TYPE ='OLD', NAME='VDAT. DAT', READONLY)

OPEN (UNIT $=22$, TYPE $=$ 'OLD', NAME $=$ 'SDAT. DAT', READONLY)

DPEN (UNIT $=20$, TYPE $=$ 'OLD', NAME $=$ 'HDAT. DAT', READONLY)

DO 87 IX $=1,8$

$N=(I X-1) * 6$

$\operatorname{READ}(24,80)$

READ (22, 80)

READ $(20,80)$

80 FORMAT (1HO)

DO 90 I $Y=1,35$

READ $(24,81) V Z(I Y, N+1), V Z(I Y, N+2), V Z(I Y, N+3), V Z(I Y, N+4)$,

$\& V Z(I Y, N+5), V Z(I Y, N+6)$

READ (22, 81) S(IY,N+1), S(IY,N+2), S(IY,N+3), S(IY,N+4),

$\& S(I Y, N+5), S(I Y, N+6)$

READ (20,81) H(IY,N+1),H(IY,N+2),H(IY,N+3),H(IY,N+4),

$\& H(I Y, N+5), H(I Y, N+6)$

81 FORMAT $(9 X, 6 F 10.3)$

90 CONTINUE

87 CONTINUE

$T G M(P)=0.9100 E+02+.4268 E+01 * P-.5102 E-01 * P * 2 .+.3531 E-03 * P * 3$.

\&-. $1287 E-05 * P * 4$. +. 2267E-08*P*5. -. 1336E-11*P*6. -. 4030E-15*P*7.

$\operatorname{VSUM}(T)=.6327 E+02-.3417 * T-.8614 \mathrm{E}-03 * T * 22$.

\&+. 4649E-05*T**3. +. 2266E-07*T*4. - $1078 E-09 * T * 5$.

\&+. $612 O E-13 * T * 6.6 .+1191 E-15 * T * 7 *$.

$P G M(T)=-.1824 E+02+.2724 * T-.6738 E-03 * T * 2$.

\&-. $2364 E-05 * T * * 3 .+2102 E-07 * T * * 4$.

\&*. $1198 E-09 * T *$ 5. $-.4317 E-12 * T * * 6$.

$\&+.4933 E-15 * T * * 7$.

$\operatorname{HSVM}(T)=.498 E+03+.4096 * T-.611 E-03 * T * 2$.

\&-. $115 E-05 * T * 3 .+211 E-07 * T * 4$.

$\&-.1835 E-09 * T * 5$. +. $6793 E-12 * T * * 6$.

$\&-.8694 E-15 * T * \# 7$.

HSLM $(T)=-.1047 E+03+.1675 E+01 * T-.1107 E-02 * T * 2$.

$\&-.3109 E-04 * T * 3 * 3 .+814 E-07 * T * * 4$.

$\&+.4744 E-09 * T * 45$. $-.226 E-11 * T * 6$.

$\&+.2589 E-14 * T * 77$.

SSUM $(T)=.9598+.8032 E-03 * T-.1172 E-04 * T * 2$.

$\&+.1973 E-07 * T * * 3 .+9589 E-10 * T * 4$.

\&-. 4041 t-1 $2 * T * 45 .+4968 E-15 * T * 26$.

\&-. $1865 E-18 * T * * 7$.

THESE CALCULATIONS FOR HIGH LOW TEMP IN HEAT PUMP

ST HELENS DATA

DATA T1, H1, ETAC/180.0,0.0,0.90/

DATA T2, H2/175. 0,552.0/

DATA TISH, HISH/295.63,600.0/

DATA TISAT, HISAT/252. 176;560.4/

DATA T3, H3/381. 44,640. O/

DATA T3A, H3A/330. $0,565.0 /$

DATA T3B, H3B/330. 0, 212.7/

DATA T4, H4/252. $176,146.6 /$

DATA T5, T6, T12/205. 0,195. 0,302.0/

DATA T15, T16, T17, TA/190.0,150.0,230.0,100.0/

DATA H12, H13/272. 8, $1182.4 /$

DATA FP, FW16, FW17/740. 8, 606. 0,47.3/

DATA RHS, WA/O. 85, 0. 04/

DATA UATOP, UABOT, UC, UE/5. 0, 1000. 0, 5. 0, 5. O/

DATA SP, CPP, BE, ICF/1. 54, 0. 45, 0.75, 2. O/

DATA HOUR, DPAIR, DPAIR6/8000.0,0.5,0.5/

DATA HPCOM, CELEC, CFUEL, CSTEAM/110. 0, 0.00001172,0.000003, 0.000004/ 
: IF ITYPE=E WE HAVE A STEAM TURBINE

REAL ICF

I TYPE $=1$

DO $2000 \quad L L=1,2$

CASE $=L L$

STATE $3 A$

$P 3 A=P G M(T 3 A)$.

$H 3 A=H S V M(T 3 A)$

$c$

.

STATE 3B

$T 3 B=T 3 A$

$P 3 B=P 3 A$

$H 3 B=H S L M(T 3 B)$

STATE 4

$T 4=T$ ISAT

$P 4=P 3 B$

$H 4=H S L M(T 4)$

c

$\mathrm{C}$

c

STATE 1

$P 1=P G M\left(T_{1}\right)$

$H 1=H S L M(T 4)$

C

c

STATE 2

$T 2=T 1$

$P 2=P 1$

H2=HSUM (T2)

$S 2=S S V M(T 2)$

C

C

c

STATE ISH

SISH $=52$

PISH $=(P 2 * P 3 A) * * 0.5$

TISH $=S H M S P(S I S H, P I S H)$

$H I S H P=H M S H(T I S H, P I S H, 2)$

$H I S H=(H I S H P-H 2) / E T A C+H 2$

C

c

STATE ISAT

PISAT $=P$ ISH

TISAT $=T G M(P I S A T)$

HISAT $=H S U M(T$ ISAT)

SISAT $=$ SSVM (TISAT)

C STATE 3

C

$P 3=P G M(T 3 A)$

$S 3 P=S I S A T$

$T 3=S H I M P P(S 3 P, P 3)$ 
$H 3 P=H M S H(T 3, P 3,2)$

C

$D F=.01$

PW5 =RH5*PG (T5)

W5 $=.622 * P W 5 /(14.7-P W 5)$

$W 6=.622 * P G(T 6) /(14.7-P G(T 6))$

$P G 6=P G(T G)$

$F W=F W 16-F W 17$

C

C

$F=0.0$

1. $F=F+D F$

c

IF (CASE. EQ. 1) $F=.05$

c

$F A 5=F W /(W 5-W 6+F * W 6-F * W A)$

FW5 $=W 5 * F A 5$

$F W 53=(1.0-F) * F W 5$

$F A 53=(1 . O-F) * F A 5$

$F W 6=F W 5-F W$

FW15=FW53-FW6

$H 6=H T P(T 6, P G(T 6))$

$H 15=T 15-32.0$

H53 $=H T P(T 5, P W 5)$

$F R 1=(F W 53 * H 53-F W 6 * H 6+F A 53 * .24 *(T 5-T 6)-F W 15 * H 15) /(H 2-H 1)$

$H 16=T 16-32$.

$H 17=T 17-32$.

WDOT $1=F R 1$ * $(H I S H-H 2) / 2545.0$

$F R 2=F R 1 *(H I S H-H 4) /(H I S A T-H 4)$

WDOT2=FR2* $(H 3-H I S A T) / 2545$. O

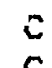

WTOT =WDOT $1+$ WDOT2

IF(CASE. NE. 1) GO TO 675

C

$c$

$P W A=W A * 14.7 /(.622+W A)$

$F W A=W A * F A \Xi * F$

HA $=H T P(T A, P W A)$

FSEXT $=$ (2545. O*WTOT+FA5*. 24*F*(TA-T5)+FW16*(H16)-FW17*H17+FP*CPP*

c

$1(T 16-T 17)-F W 5 * H 53 * F-F W 15 * H 15+F W A * H A$

)$/(H 13-H 12)$

GO TO 3

C

C

675 CONTINUE

WTOT $1=-(F A 5 * .24 * F *(T A-T 5)+F W 16 * H 16-F W 17 * H 17+F P * C P P *(T 16-T 17)-F W 5 *$ $1 H 53 * F-F W 15 * H 15) / 2545$.

IF (WTOT1. GE. WTOT) GO TO 2

GO TO 1

IF (ABS (WTOT1-WTOT). LE. O. OO1*WTOT) GO TO 5 
$F=F-D F$

$D F=D F / 10.0$

GO TO 1

CONTINUE

FSEXT $=0.0$

CONTINUE

DTE $=(T G(P W 5)+T 15) * .5-T 1$

$Q E=F R 1 *(H 2-H 1)$

$A E=Q E /(U E * D T E)$

COSTE $=A E * .3$

VE $=A E / 2000$.

$F R A=F A 53 /(F W 6+F A 53)$

$X 7=((1 .-F R A) / 18) /.((1 .-F R A) / 18 .+F R A / 28.93)$

$P W 7=X 7 * 14.7$

$F R 2 P=F R 2 *(H 3-H 3 B) /(H 3 A-H 3 B)$

DTABOT $=T 3 A-T 12$

QABDT $=F R 2 P *(H 3 A-H 3 B)$

$A A B O T=Q A B O T /(U A B O T * D T A B O T)$

$C A B O T=A A B O T * 10.0$

VABOT $=A A B O T / 20.0$

$Q C=F R 2 *(H 3 B-H 4)$

$\mathrm{T} 7=\mathrm{T} 6$

$\mathrm{DT} 7=5.0$

4

$T 7=T 7+D T 7$

$Q C 1=F A 53 * .24 *(T 7-T 6)+F W 6 *(H T P(T 7, P W 7)-H 6)$

IF $(Q C 1 . G E . Q C)$ QD TO 6

C

GO TO 4

IF ( $A B S(Q C 1-Q C)$. LE. $0.001 * Q C)$ GO TO $B$

$T 7=T 7-D T 7$

$D T 7=D T 7 / 10.0$

c

GO TO 4

CONT INUE

$H 7=H T P(T 7, P W 7)$

$D T C=((T 3 A+T 4)-(T 6+T 7)) * .5$

$A C=Q C /(U C * D T C)$

$\operatorname{CoSTC}=A C * .3$

$V C=A C / 2000.0$

CCOMP $=$ HP COM $* W T O T$

$C P M=W T O T * 2 O$.

c

IF (ITYPE. EQ. 2) CPM $=W T O T * 90$.

$\mathrm{C}$

$C T O T=C A I O P+C A B O T+C O S T C+C O S T E+C C O M \Gamma I C P M$

$T=T 6+460$.

RHOG $=P G(T 6) * 144.0 /(1545.0 * T / 18.0)+(14.7-P G(T 6)) * 144.0 /(53.3 * T)$

HPFAN $=($ FA53+FW6) $* 144 . /($ RH06*778. *2545.)

FSTEAM $=(Q A B Q T) /(H 13-H 12)$

DHBOIL $=H 13-H 12$

QBOIL $=D H B D I L * F S T E A M / B E$

QPM $=W T O T * 2545 . /(.3 * .95)$

C

IF(ITYPE. EQ. 1 ) GO TO 11

C 
PMST=WTOT*2545. /(385. *.65)

$Q P M=P M S T * D H B O I L / B E$

GO TO 12

11 CONTINUE

$P M S T=0$

C

12 CONTINUE

HPFANG $=H P F A N * D P A I R G$

$E R=Q B O I L+Q C-Q P M-H P F A N 6 * 2545$.

$E R 2=S P * F P * D H B O I L / B E-Q P M-H P F A N 6 * 2545$

FA5THE $=F W /(W 5-W A)$

FW5THE $=$ W5 *FA5THE

FWATHE=FASTHE*WA

QTHE=FA5THE*. 24* (T5-TA) +FW5THE*H53-FWATHE*HA

$\therefore+F P * C P P *(T 17-T 16)+F W 17 * H 17-F W 16 * H 16$

$S P T=Q T H E /(D H B O I L * F P)$

$E R 3=Q T H E / B E-Q P M-H P F A N 6 * 2545$.

C

IF (CASE. NE. 1$)$ GD TO 766

$c$

$B B B=F S E X T *(H 13-H 12) / B E$

$E R=E R+B B B$

$E R 2=E R 2+B B B$

$E R 3=E R 3+B B B$

766 CONTINUE

C

IF(ITYPE. EQ. 2) FCOST=WTOT*2. 545*350.*24. *CSTEAM/1000.

C

IF(ITYPE. EQ. 1) FCOST=WTOT*. 7457*24.*350.*CELEC/(1000.*. 95)

SCDST $=S P * F P * 350 . * 24 . * C S T E A M / 1000$.

SCOST2=SP*FP*DHBOIL*24. *350. *CFUEL/ (BE*1. OE6)

$\mathrm{C}=4.0 / 3.14$

$T=T 5+460.0$

$R H O 5=(14.7-P W 5) * 144.0 /(53.3 * T)+P W 5 * 144.0 /(85.8 * T)$

$F 5=F A 5+F W 5$

$V 5=30.0 * 3600.0$

$A S=F 5 /(R H O S * V S)$

$D S=S Q R T(C * A S)$

$F G=F A 5 *(1.0-F)+F W G$

$V G=30.0 * 3600.0$

$A G=F G /(R H D G * V G)$

$D G=S Q R T(C * A G)$

RHO7 $=$ RHO6* $(T 6+460.0) /(T 7+460.0)$

$V 7=30.0 * 3600.0$

$A 7=F 6 /(R H O 7 * V 7)$

$D 7=\operatorname{SQRT}($ ( *A" )

RHO1=1. $0 / 2.6$

$V 1=15.0 * 3600.0$

$A 1=F R 1 /($ RHO1 \#V1)

$D 1=S Q R T(C * A 1)$

RHO2 $=1$. O/VSUM(T2)

$V E=18.0 * 3600.0$

$A 2=F R 1 /(R H D 2 * V 2)$

$D E=S Q R T(C * A E)$

RHOISH $=1.0 / V M S H(T I S H, P I S H)$

$V I S H=18.0 * 3600.0$

AISH $=F R 1 /($ RHOISH*VISH) 
DISH=SQRT (C*AISH)

RHOIST $=1$. O/VSVM (TISAT)

VISAT $=18.0 * 3600.0$

AISAT =FRE/ (RHOIST*VISAT)

DISAT $=S Q R T(C * A I S A T)$

RHO3 $=1.0 / V M S H(T 3, P B)$

$V 3=18.0 * 3600.0$

$A 3=F R 2 /(R H D 3 * V 3)$

$D 3=S Q R T(C * A 3)$

RHO3A=1. O/VSVM (T $3 A$ )

$V 3 A=18.0 * 3600.0$

$A 3 A=F R 2$ ( $\{$ RHO3A*V3A)

$D 3 A=S Q R T(C * A 3 A)$

RHO3B $=1.0 / 0.026$

$V 3 B=10.0 * 3600.0$

$A 3 B=F R 2 /(R H O 3 B * V 3 B)$

$D 3 B=S Q R T(C * A 3 B)$

$\mathrm{RHO} 4=1.0 \% .024$

$V 4=10.0 * 3600.0$

A4=FR2 $/($ RHO4*V4)

$D 4=S Q R T(C * A 4)$

$V I N 1=(1.0 / R H O 2) * F R 1$

VOUT $1=(1.0 /$ RHOISH $) * F R 1$

VINE $=(1.0 /$ RHOIST $) * F R 2$

VOUTE $=(1: 0 / R H O 3) * F R 2$

C

IF(ITYPE. EQ. 1) GO TO 25

C

C

VSTM $=30.0 * 3600.0$

RHOSTM $=1.0 / 1.032$

ASTM $=P M S T /($ VTM $* R H O S T M)$

$D B=S Q R T(C * A S T M)$

25 CONTINUE

RHD12=58. 8

RHD 1 $3=.1$

$V 12=30.0 * 3600.0$

$V 13=10.0 * 3600.0$

A12=FSTEAM/ (RHO12*V12)

A13=FSTEAM/ $($ RHO13*V13)

D12=SQRT $(C * A 12)$

D 1 3=SQRT $(C * A 13)$

DPSTM $=288.0$

FANHP $=F 5 * D P A I R * 144 /(R H 05 * 778.0 * 2545.0)$

PUMPHP =FSTEAM *DPSTM/ (RHO12*778. O*2545. O)

EPRE $=S P * F P * H D U R * D H B O I L / B E$

$E F U T=(Q P M+(F A N H P+H P F A N G) * 2545 / 0.3) * H O U R$

ESAVE $=E P R E-E F U T$

C

c

QPMA=WTOT*2つ45

C

IF (ITYPE. EQ. 1) COSTF $=($ QPMA $+($ HPFANG $+F A N H P) * 2545) *$ HOUR *CELEC

IF (ITYPE. EQ. 2) COSTF $=($ QPM*CFUEL+(HPFANG+FANHP )*2545*CELEC )*HDUR COSTP=EPRE $\#$ CFUEL.

FIRSTC $=$ CTOT $*$ I CF

$R O I=(\operatorname{COSTP}-\operatorname{COSTF}-F I R S T C / 10) / F I R S T C$

C

$C D P=(Q A B D T+Q C) /(W T D T * 2545)$

C

$P P=F I R S T C /(C O S T P-C O S T F)$ 
WR ITE $(6,20)$

WRITE $(6,21)$

WR I TE ( 6,22$)$ T 1, T ISH, TISAT, T3

WRITE ( 6,23$)$ T3A, T4, T5, RH5

WRITE (6, 24) TG, T7, F

WRITE $(6,42)$

WRI TE $(6,26)$ CTOT, FIRSTC

WR I TE $(6,27)$ COSTP, COSTF

WR I TE (6, 28) ROI, PP

WRITE ( 6,29$)$ EPRE, EFUT

WR I TE $(6,30)$ ESAVE

WRITE $(6,31)$

WR I TE $(6,32)$ WDOT 1, FR 1

WRITE $(6,33)$ VIN1, VOUT 1

WRITE (6, 34) P2, P ISH

WR I TE $(6,35)$ T2, TISH

WRITE $(6,44)$

WR I TE (6, 32) WDOT2, FRE

WRITE $(6,33)$ VIN2, VDUT2

WR I TE (6, 34 ) P ISAT, P3

WR I TE $(6,35)$ T ISAT, T3

WR I TE (6, 47) WTOT, CCOMP, COP

WR I TE $(6,39)$

WR I TE (6, 38) QABOT, DTABOT, AABOT, VABOT, CABOT

WR I TE $(6,41)$

WRITE $(6,38) Q C, D T C, A C, V C, C D S T C$

WR I TE $(6,43)$

WR I TE (6, 38) QE, DTE, AE, VE, COSTE

WRI TE $(6,45)$

WR I TE (6, 46) D 1, D2, DISH, DISAT, D3, D3A, D3B, D4, D5, D6, D7, D12, D13

FORMAT (1HO, $Q=\prime, E 13.4, ' D T=', F 5.1$,' $A R E A=', F 10.1$,' VOLUME=', 
FORMAT (/1HO, 28X, 'LINE SIZES'/)

FORMAT ( 1 HO, 'D 1=', FB. 3,' D2=',FB. 3,' DISH=', F8. 3,' DISAT=', FB. 3/

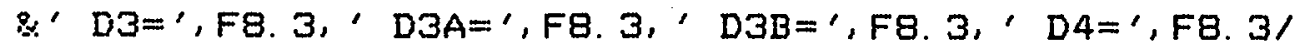

2, DS=',F8. 3,' DS=',FB. 3,' D7=',FB. 3'

$\mathrm{C}^{\prime} \mathrm{D} 1 \mathrm{Z}={ }^{\prime}, \mathrm{FB} .3 \mathrm{\prime}^{\prime} \mathrm{D} 13=\mathrm{\prime}^{\prime}, \mathrm{FB} .3$ )

2000 CONTINUE

STOP

END

FUNCTION $\operatorname{HTP}(T 1, P 1)$

$T=255.38+(T 1) / 1.8$

$P=P 1 / 14.6959$

$B 1=(2641.62 * 10 . *(80870.0 /(T * T))) / T$

$B O=1.89-B 1$

$B 2=82.546$

$B 3=162460.0 / T$

$B 4=.21828 * T$

$B 5=126970.0 / T$

$F O=1.89-B 1 *(372420.0 /(T * T)+2.0)$

$B G=B O * B 3-2 . O * F O *(B 2-B 3)$

$B 7=2$. $O * F O *(B 4-B 5)-B O * B 5$

$B 8=.4342944 * A L Q G(T)$

$F=775.596+(.63296+.0001624 * T) * T+47.3635 * B 8$

$B 9=B O * P * P /(2.0 * T * T)$

$H T P=F+.043557 *(F O * P+B 9 *(B O *(B 2-B 3+2.0 * B 7 * B 9)-B 6))$

RETURN:

END

FUNCTION TG(P)

IF (P. GT. 11.526) GO TO 1

$T G=38.795885+102.3232 * P \ldots 53.76781 * P * 2+17.63585 * P * 3 .-3.338111 *$

$* P * 4 .+.3567123 * P * 5 .-1.994707 E-2 * P * 6 .+4.5293 E-4 * P * 7$.

GO TO 2

$1 T G=147.4684+5.665215 * P-.1045811 * P * 2 * 2 .+1.21192 E-3 * P * * 3$.

* $\quad-7.2 E-6 * P * 4 .+2 . O E-8 * P * 5$.

$\because$ CONTINUE

RETURN

END

C

$\mathrm{C}$

$\mathrm{C}$

\section{FUNCTION PG(T)}

DIMENSION $F(B)$

DATA $F /-741.9242,-29.721,-11.55286,-.8685635$,

\&. 1094098, . 439993, . 2520658, .05218684/

TFAR $=T$

$A=0.0$

TC $=5.19 . *$ (TFAR-32. )

$T A U=1000 . /(T C+273.15)$

PCRIT $=220.88 * 14.5$

TCR I T $=374.1 .36$

DO $20 \quad J=1,8$

$A=A+F(J) *(.65-.01 * T C) *(v-1)$

20 CONTINUE

$B=T A U *(1 . / 10 . * * 5) *.(T C R I T-T C)$.

$C=A * B$ 
$P G=P C R I T * E X P(C)$

10 CONTINUE

\section{RETURN}

END

FUNCTION SHMSP (SI, PI)

DATA P/14.7, 15. 2, 18. 7, 20., 23. , 28. 1, 30., 34. 1, 40. , 41.1, $849.1,50 ., 58.4,60 ., 69 ., 70 ., 80,81 ., 90 ., 95 .$, $\$ 100,110,120,127,130,140,147,150,160$, \&169, 170, 180, 190., 194., 200, 210, 220, ,221, 230, , $\& 240 ., 250,251 ., 260,270,280,284 ., 290 \ldots, 300 . /$

DATA T/150, , 160, 170., 180, 190, ,200, 210, ,221.,230., \&240, , 250., 253. , 260., 270. , 280, , 281., 290.,

\&300. , 302. , 310. , 320. , 330. , 334. , 340., 350.,

8360, 370, 380, 390. , 400, 410, 420.;

\&30., 440.,450.

DIMENSION T(35), P(48), ST (35).

COMMON/PAUL/ $S(35,48)$

DO $10 J=1,48$

IF (PI. LE. $P(J+1))$ GOTO 15

10 CONTINUE

15 DO $20 \quad I=1,35$

$Q 1=(P(J)-P(J+1)) /(P(J)-P I)$

$A=S(I, N)$

$B=S(I, J+1)$

$S T(I)=A-((A-B) / Q 1)$

20 CONTINUE

DO $25 \quad J=1,35$

IF(SI. LE. ST $(J+1)$ ) GOTO 30

25 CONTINUE

$30 Q 2=(5 T(v)-5 T(v+1)) /(\operatorname{ST}(u)-5 T)$

$A=T(J)$

$B=T(j+1)$

$S H M S P=A-((A-B) / Q 2)$

RETURN

END

FUNCTION HMSH(TI, PI, IF)

DIMENSION T(35), P (48)

COMMON/TOM/ $\mathrm{H}(35,48)$ 
DO $10 \quad I=1,35$

IF (T (I). GE. TI) GOTO 15

10 CONT INUE

15 DO $20 \quad J=1,48$

IF (P(J). GE.PI) GOTO 25

20 CONTINUE

$25 A=H(I-1 ; J-1)$

$B=H(I-i, J)$

$C=H(I, J-1)$

$D=H(I, J)$

$Q 1=(T(I-1)-T(I)) /(T(I-1)-T I)$

$X 1=A-((A-C) / Q 1)$

$X 2=B-((B-D) / Q 1)$

$Q 2=(P(J-1)-P(J)) /(P(J-1)-P I)$

$H M S H=X 1-((X 1-X 2) / Q 2)$

DATA P/14.7, 15.2, 18. 7, 20., 23., 28. 1, 30., 34.1,40., 41.1,

\&49. 1, 50.,58. 4,60.,69.,70, 80, 81, 90.,95.,

$\$ 100,110,120,127,130,140,147,150,160 \ldots$

\&169., 170, 180, 190, 194.,200, 210, 220, 221., 230.,

\&240. , 250, , 251., 260, , 270, 280, 264., 290, 300. /

DATA T/150.,160.,170, 180., 190, , 200, 210, 221, ,230.,

\&240., 250., 253., 260, , 270., 280., 281.,290.,

\&300. 302., 310, 320., 330, 334, 340, 350.,

$\& 360 ., 370$, 380., 390. , 400, 410., 420. .

\&430. , 440., 450. I

RETURN

END

FUNCTION VIMSH (TI,PI)

DIMENSION T(34), P(48)

COMMON/GLEN/VZ $(35,48)$

DO $10 \quad I=1,34$

IF (T(I). GE. TI) GOTO 15

10 CONTINUE

$15 D O 20 \quad J=1,48$

IF (P(J). GE.PI) GOTO 25

20 CONTINUE

25 $A=V \angle(I-1, J=1)$

$B=V Z(I-1, J)$

$C=V Z(I, J-1)$

$D=V Z(I, J)$

$Q 1=(T(I-1)-T(I)) /(T(I-1)-T I)$

$X 1=A-((A-C) / Q 1)$

$X 2=B-((B-D) / Q 1)$

$Q 2=(P(J-1)-P(J)) /(P(J-1)-P I)$

$V M S H=X 1-\left(\left(X_{1}-x_{2}\right) / Q 2\right)$

DATA P/14. 7, 15.2, 18. 7, 20., 23. , 28. 1, 30., 34.1,40.,41.1, \&49.1,50.,58. 4,60.,69.,70.,80.,81.,90.,95.,

$\& 100,110,120,127,130,140,147,150,160 \ldots$,

\&169, 170, 180, 190, 194.,200, 210, 220, 221.,230.,

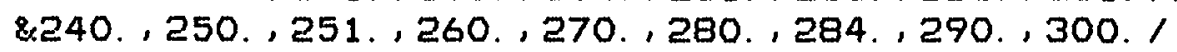

DATA T/150., 160.,170, 180., 190, , 200., 210., 221, , 230.,

\&240., 250., 253. , 260. , 270. , 280. , 281., 290.,

\&302., 310., 320., 330., 334. , 340., 350.,

$8360,370,380,390,400,410,420$,

$\& 430$, 440., 450. I

RETURN

END 


\section{OPTIMIZATION (ELECTRIC MOTOR)}

Having represented the heat pump system performance numerically in the previous section it is now necessary to determine the optimum operating conditions (eg. state point specifications). The problem of optimization can be thought of in this case, as a complex mathematical equation ( $f$ ) with the quantity to be optimized $(\psi)$ on the left hand side of the equals sign.

$$
\psi=f\left(a_{1}, a_{2}, a_{3} \ldots a_{n}\right)
$$

In the general case each variable $\left(a_{1}, a_{2}, a_{3} \ldots a_{n}\right)$ which appears in the equation is independent of every other variable and an optimization would involve selection from among $\left(n^{2} * z\right)$, cases where $z$ is the number of values each variable may assume and $\eta$ is the number of variables. Fortunately it is often the case that several variables will be found to be dependent rather than independent and thus the total \# of independent variables will be reduced.

The heat pump simulation involves over fourty (40) variables and it is easily seen that the number of possible cases (for even small z) gets large. With the apprupriate equations of constraint however, it is possible to reduce this unwieldy number of variables from fourty $(40)$ to three (3); those which are actualiy independent. These three (3) independent variables are (see Figure 4.1 ):

T3A - the compressor's 2nd stage discharge temperature (stream = methanol)

T1 - the evaporator's tube side inlet temperature (stream = methanol)

T6 - the evaporator's shell side exhaust temperature (stream = supply of wet air) 


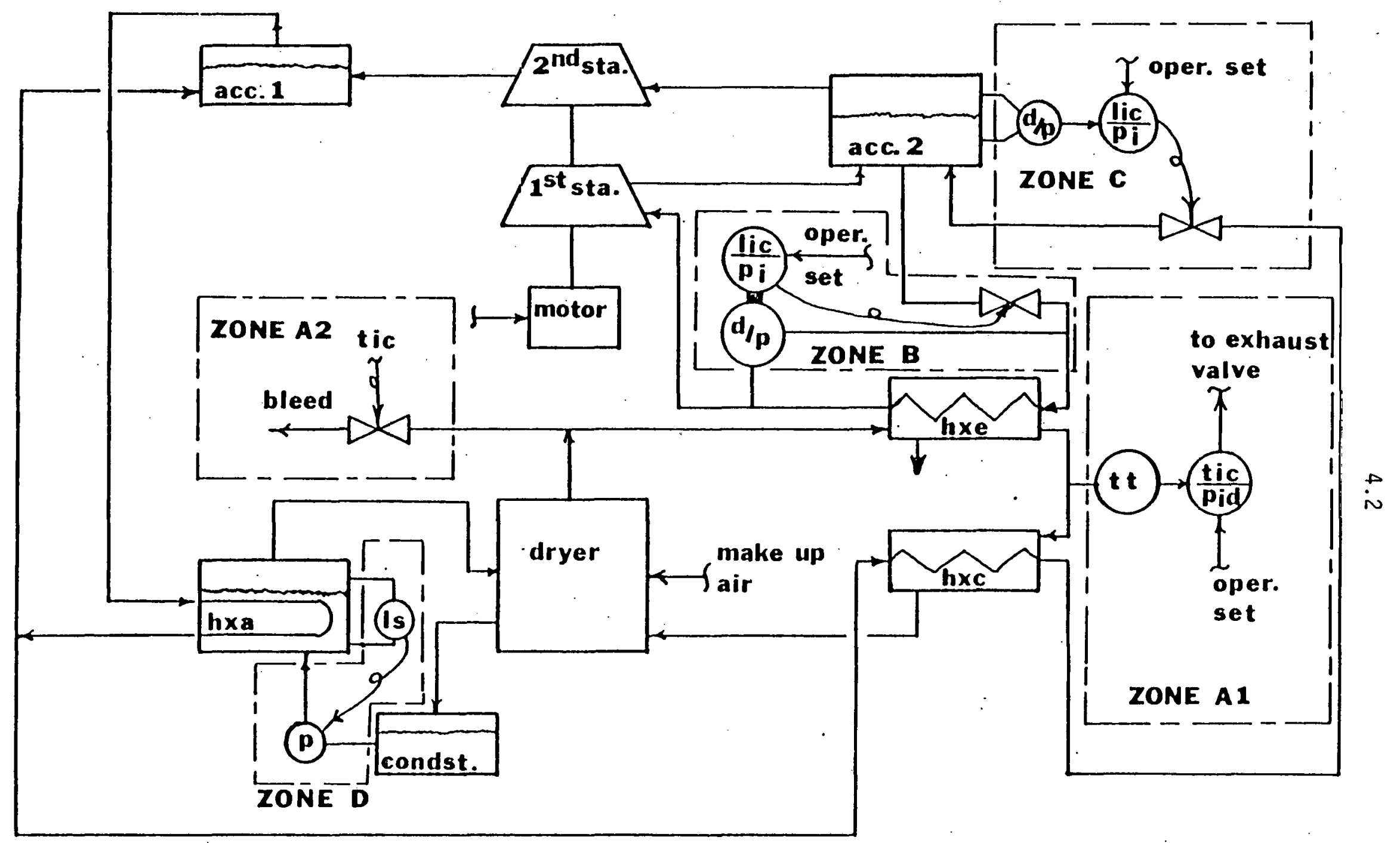

FIGURE 4.1 CONTROL SYSTEM 


\section{3}

Thus far the quantity to be optimized, $\psi$, has not been identified. It can be selected based either on performance or economics, but not in general or both. That is to say that the conditions required for optimum performance (eg. maximum energy savings) will not necessarily. coincide with those conditions required for optimum economy (best return on investment). For this reason studies in the form of parametric maps have been made based on each of these optimization parameters:

$\psi_{1} \equiv$ return on investment (economic optimization)

$\psi_{1} \equiv$ energy savings (performance optimization)

The parametric maps in this section (Figure 4.2 through Figure 4.29 ) are divided into two sets, those for the demonstration model and those for the industrial model. Since a discussion of either set of maps will cover essentially the same points, only those maps pertaining to the demonstration model will be discussed in the following paragraphs.

Referring to Figure 4.2 note that T3A is fixed at $315^{\circ} \mathrm{F}$ and ROI is plotted versus $\mathrm{Tl}$ for three values of $\mathrm{T} 6\left(192^{\circ} \mathrm{F}, 194^{\circ} \mathrm{F}, 196^{\circ} \mathrm{F}\right)$. The most obvious feature of this graph is the falling ROI with increasing $T 7$. By noting that $T$ is the inlet methanol temperature to the evaporator (HXE) and that the methanol is used to cool the dryer exhaust (see Figure 4.1) and thus condense out water, a better understanding of this phenomenon is gained. In essence, as $T T$ increases the amount of water condensing in (HXE) decreases and for this reason the heat exchanger HXE gets inordinately large in order to compensate for the reduced heat transfer potential. Another pronounced feature of the graph is the sharp fall in ROI with increasing values of $T 6$. The rationale for this is that as T6 increases so does the specific humidity at state six (6). Since water is evaporating at a fixed 
rate in the dryer the air flow must be sufficient to carry the water vapor: increasing $T 6$ decreases the capacity of the air to carry the water and the air mass flow rate must increase requiring the fan size to increase. At this point it is important to point out that some variation of the three independent variables $(T 3, T T, T 6)$ from optimum is inevitable in the real world and therefore optimum operating conditions should be achievable over a fairly broad range ( $\left.2 \mathrm{OF}^{-} 5^{\circ} \mathrm{F}\right)$ of each of these three variables. With this in mind it is evident from the graph that $\mathrm{Tl}$ between $175^{\circ} \mathrm{F}$ and $179^{\circ} \mathrm{F}$ and $\mathrm{T} 6$ between $192^{\circ} \mathrm{F}$ and $194^{\circ} \mathrm{F}$ offers a realistic optimum with an ROI at about 100\%/year. Viewing Figures 4.3 through 4.5 indicates that ROI is not particularly sensitive to $T 3 \mathrm{~A}$ between $315^{\circ} \mathrm{F}$ and $330^{\circ} \mathrm{F}$.

Figures 4.6 through 4.8 were generated using the same data as the previous three graphs, but hold $\mathrm{Tl}$ constant instead of T3A. These plots emphasize again the importance of maintaining T6 between $192^{\circ} \mathrm{F}$ and $194^{\circ} \mathrm{F}$. In addition the figures indicate only small differences in ROI for $\mathrm{Tl}$ between $170^{\circ} \mathrm{F}$ and $185^{\circ} \mathrm{F}$.

The next seven yraphis (Figures 4.9 through 4.15 ) arc based on the second optimization parameter, performance as indicated by energy savings. From Figure 4.9 it is seen that both $T 6$ and $T 1$ have a pronounced affect on performance. Energy savings are much more sensitive to these values than ROI and thus holding optimum operating conditions based on performance wi.11 require a much more sophisticated and sensitive control system. Viewing Figures 4.10 through 4.12 indicates that energy savings are affected by varying $T 3 A$ although not nearly so much as by $T 7$ and $T 6$. Optimum operating conditions for this case are $\mathrm{T} 1$ between $189^{\circ} \mathrm{F}$ and $190^{\circ} \mathrm{F}$, T6 between $191^{\circ} \mathrm{F}$ and $192^{\circ} \mathrm{F}$, and $T 3 A$ between $315^{\circ} \mathrm{F}$ and $316^{\circ} \mathrm{F}$. Figures 4.13 through 4.15. were generated using the same data as the previous three graphs, but 


\section{5}

holding $T 1$ constant instead of T3A. These plots clearly show the increased sensitivity of energy savings to varying T3A.

These parametric maps for the demonstration model are essential in order to visualize trends in optimization from both an economic and an efficiency standpoint. From this analysis it has been possible to specify the three parameters T3A, TT, and T6 for optimum conditions. The investigators have chosen their optimum conditions based on economics as they judge their industrial counterparts a.lso will. 
Figure 4.2. Heat Pump Performance Based on Return on Investment

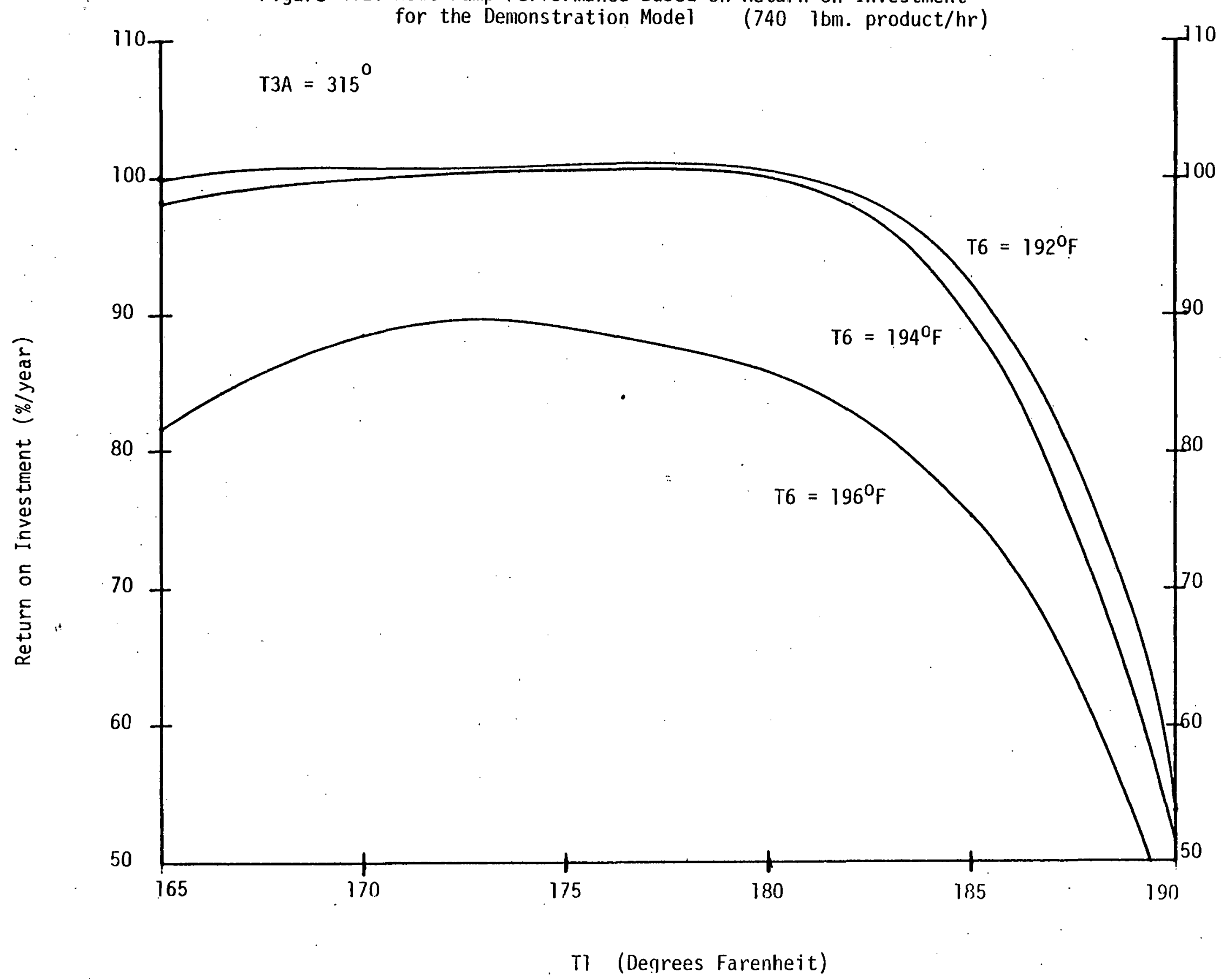


Figure 4.3. Heat Pump Performance Based on Return on Investment for the Demonstration Model (740 $\mathrm{lbm}$. product/hr.)

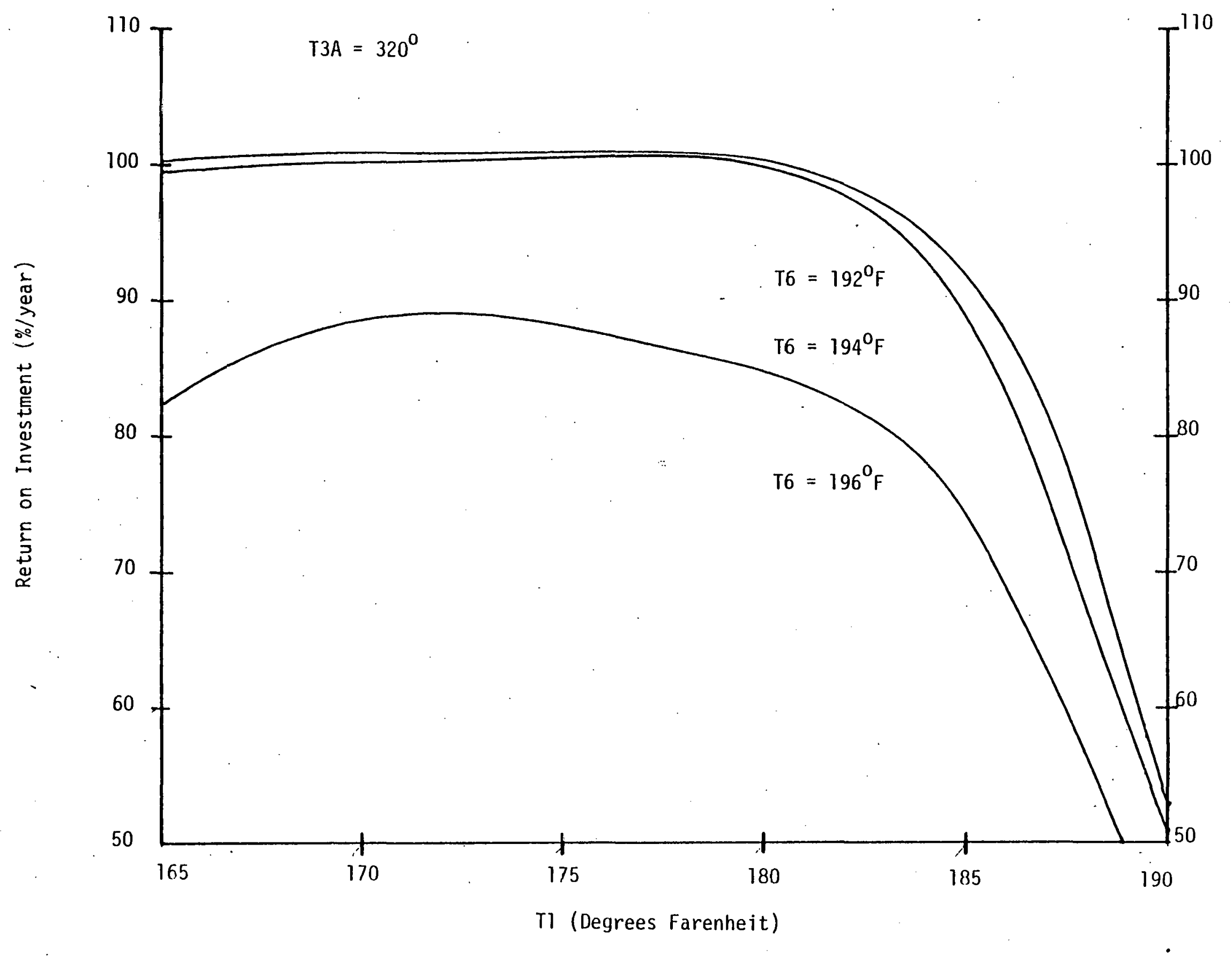


Figure 4.4. Heat Pump Performance Based on Return on Investment for the Demonstration Model (740 1bm. product/hr)

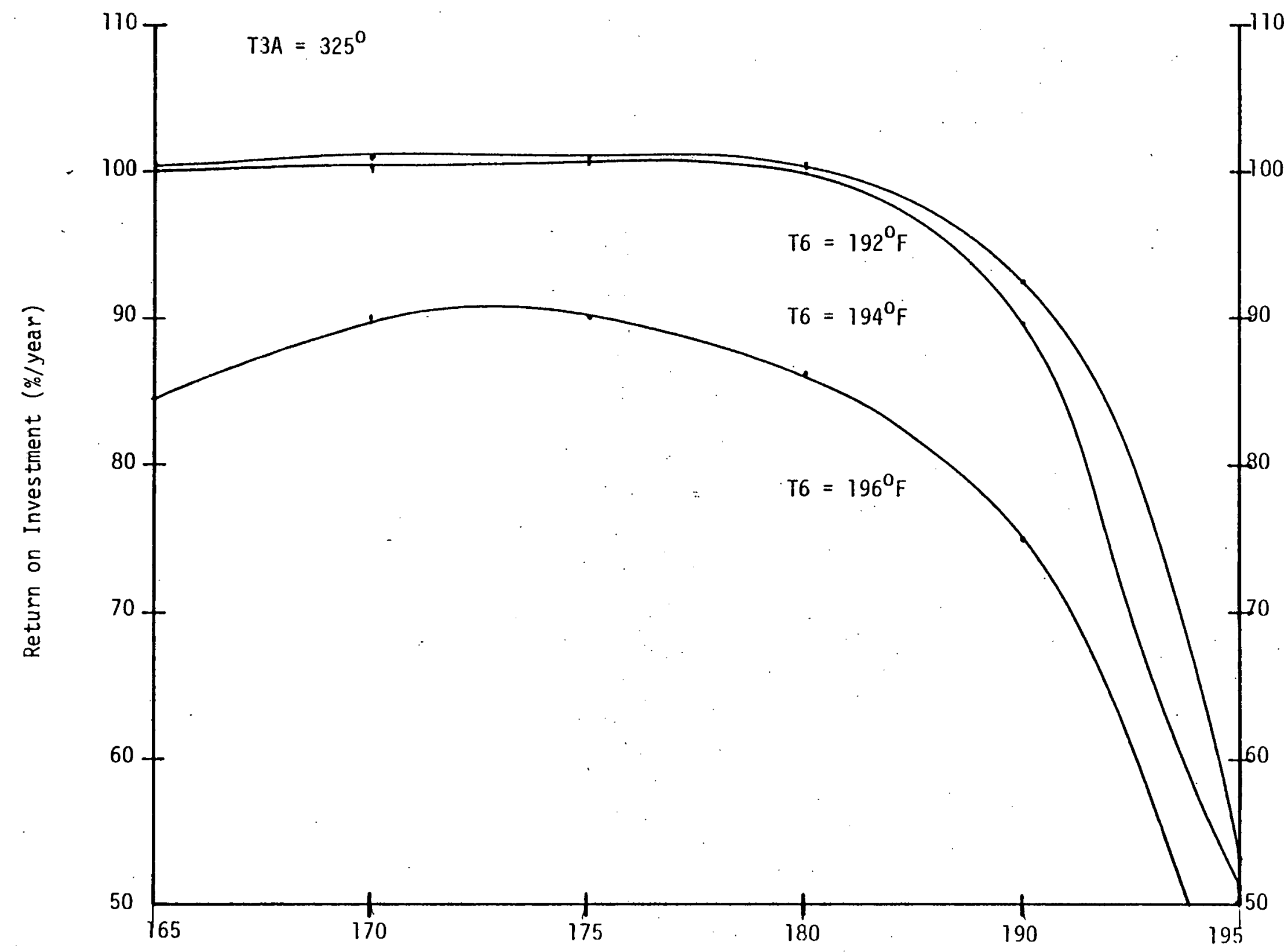

Tl (Degrees Farenheit) 
Figure 4.5. Heat Pump Performance Based on return on Investment for the Demonstration Model (740 lbm. product/hr)

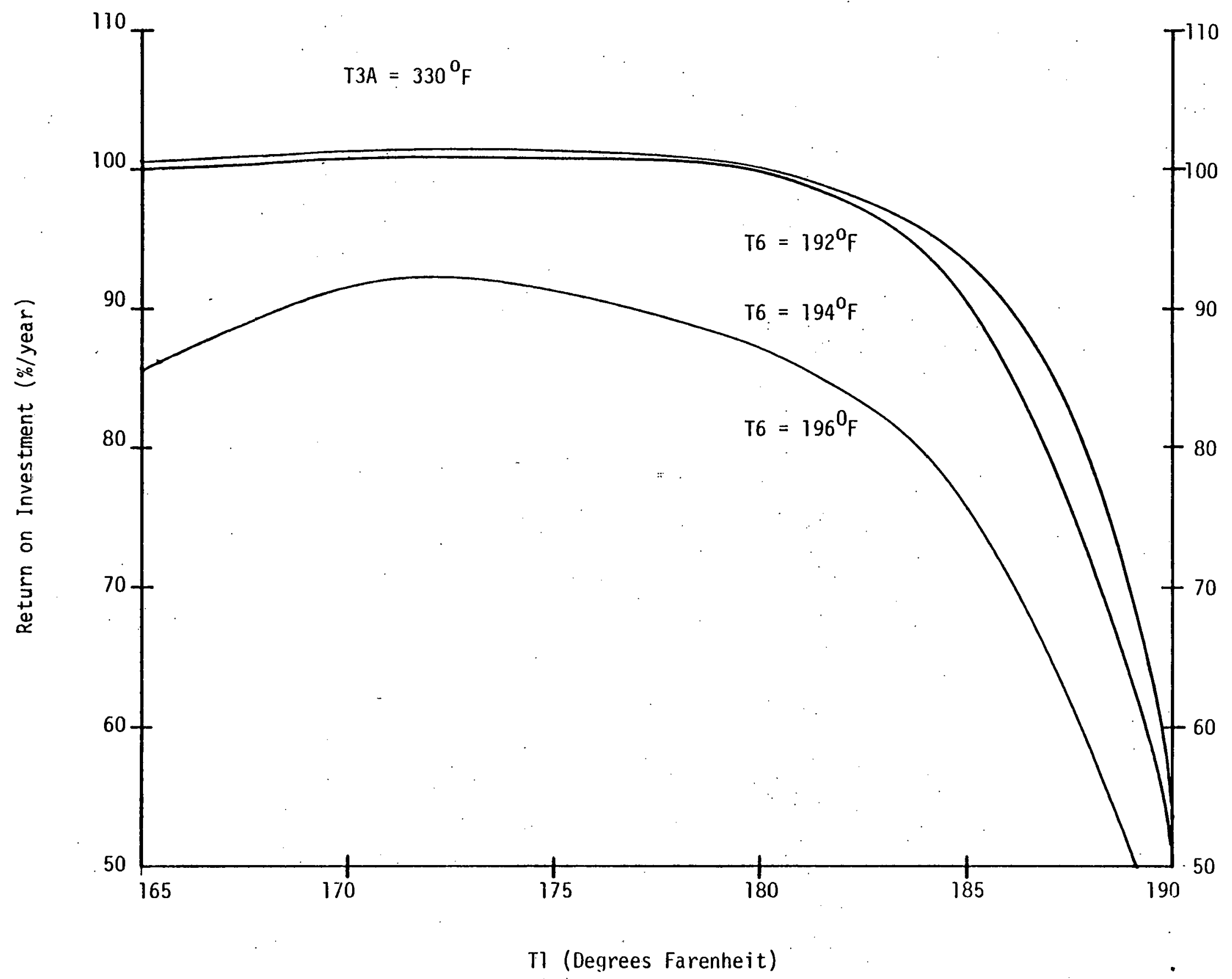


Figure 4.6. Heat Pump Performance Based on Return on Investment for the Demonstration Model (740 lbm product/hr)

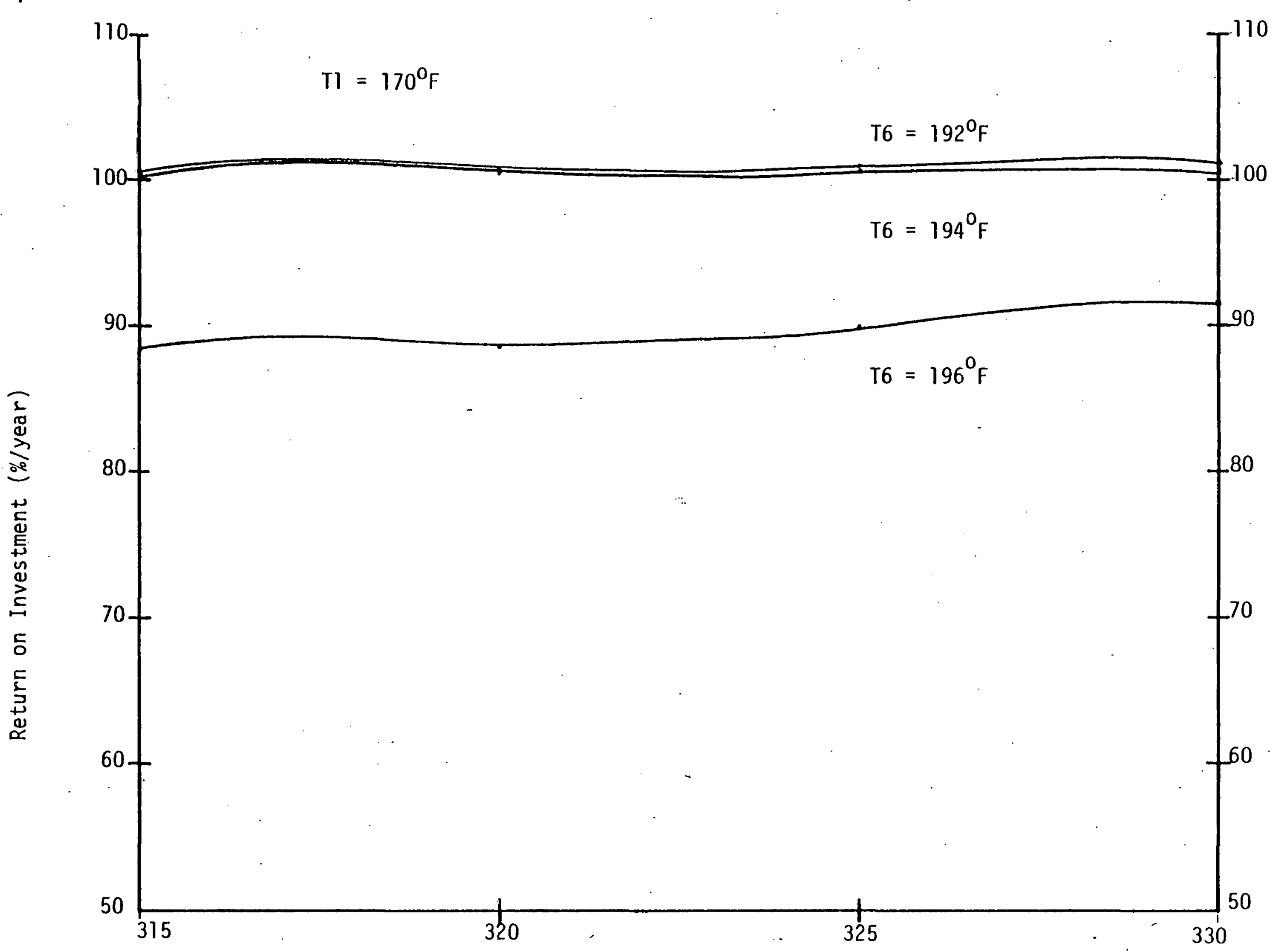

T3A (Degrees Farenheit) 
Figure 4.7. Heat Pump Performance Based on Return on Investment for the Demonstration Model (740 1bm. product/hr)

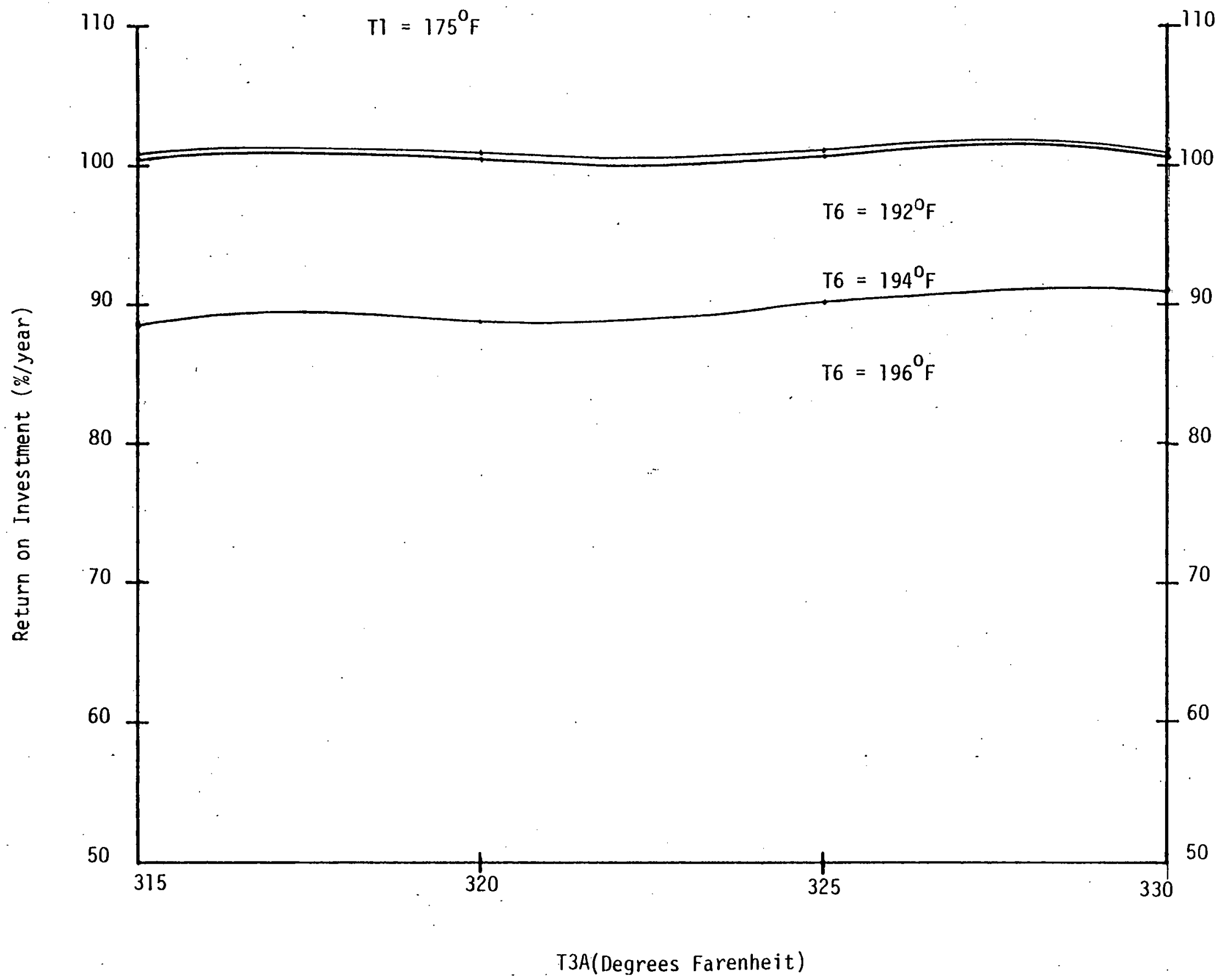


Figure 4.8. Heat Pump Performance Based on Return on Investment for the Demonstration Model (740 1bm. product/hr)

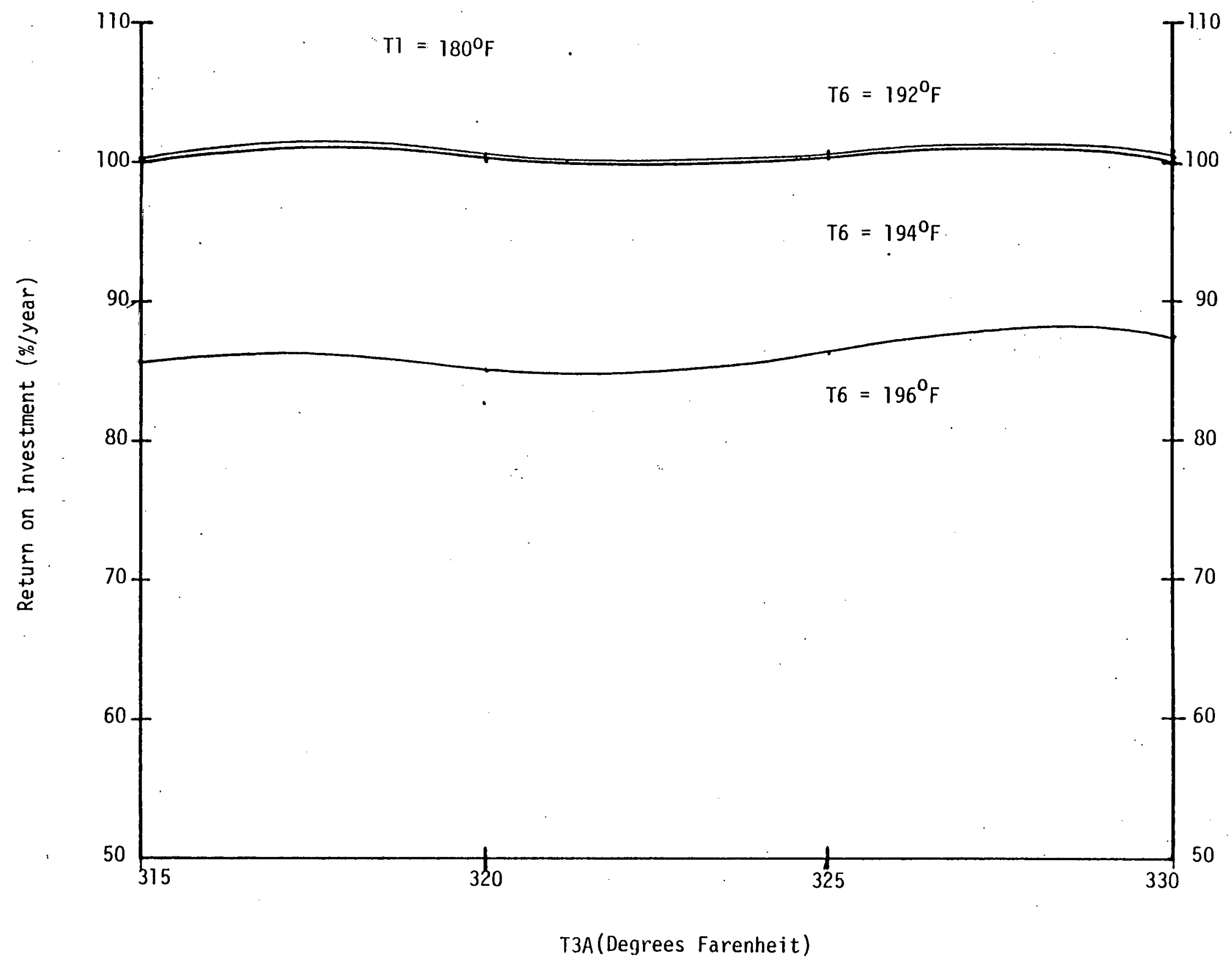


Figure 4.9. Heat Pump Performance Based on Energy Saved For the Demonstration Model

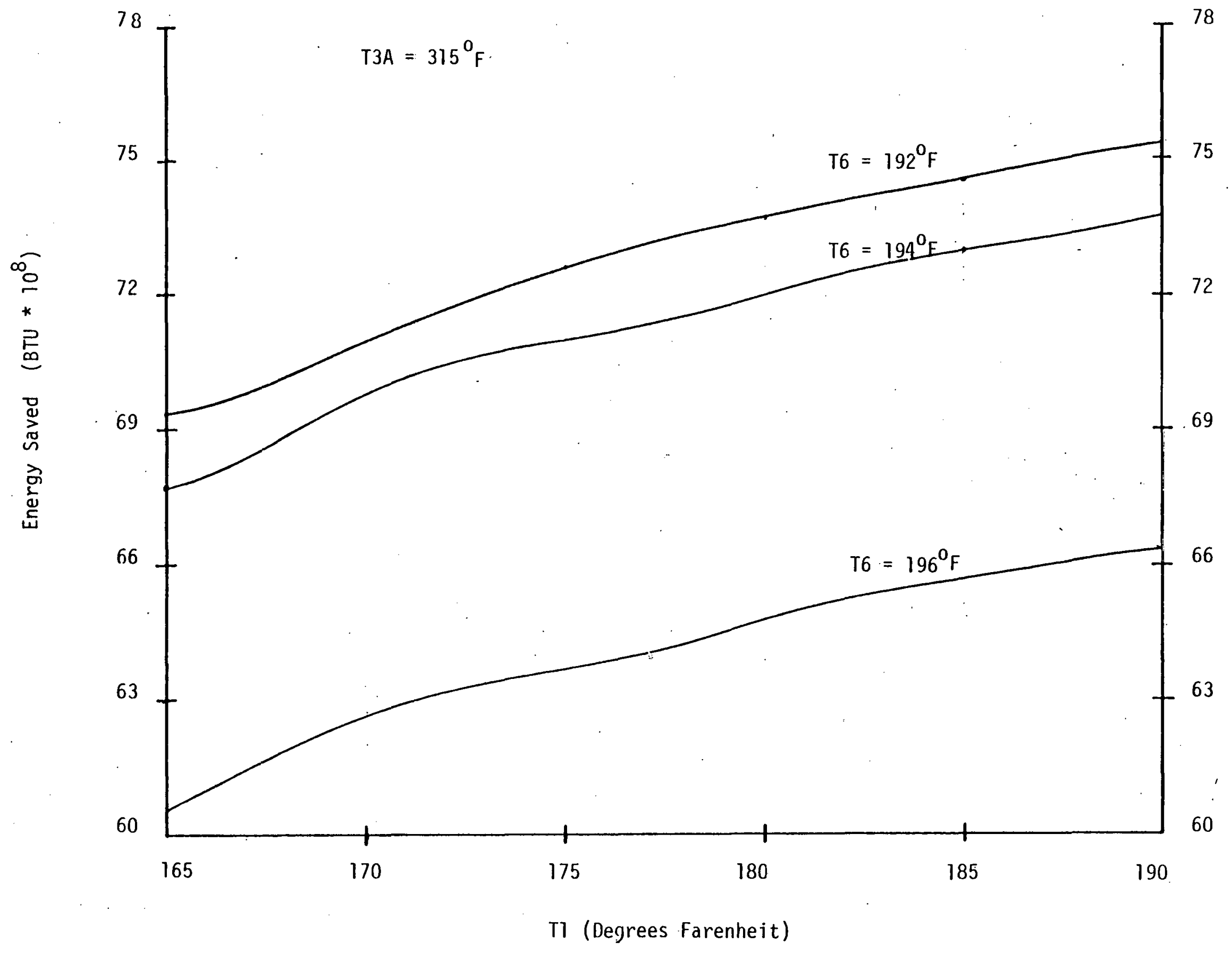


Figure 4.10. Heat Pump Performance Based on Energy Saved For the Demonstration Model

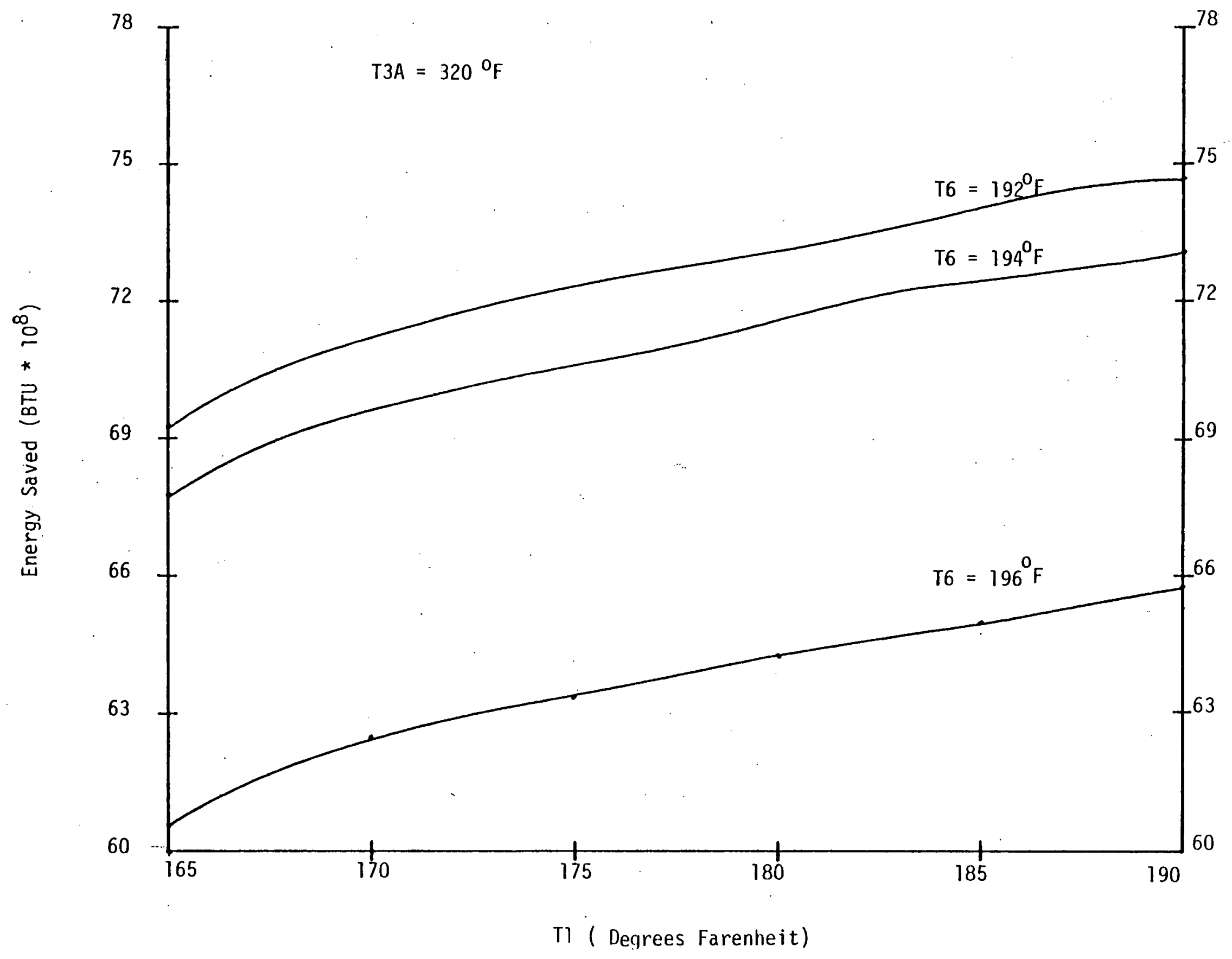


Figure 4.11. Heat Pump Performance Based on Energy Saved For the Demonstration Model

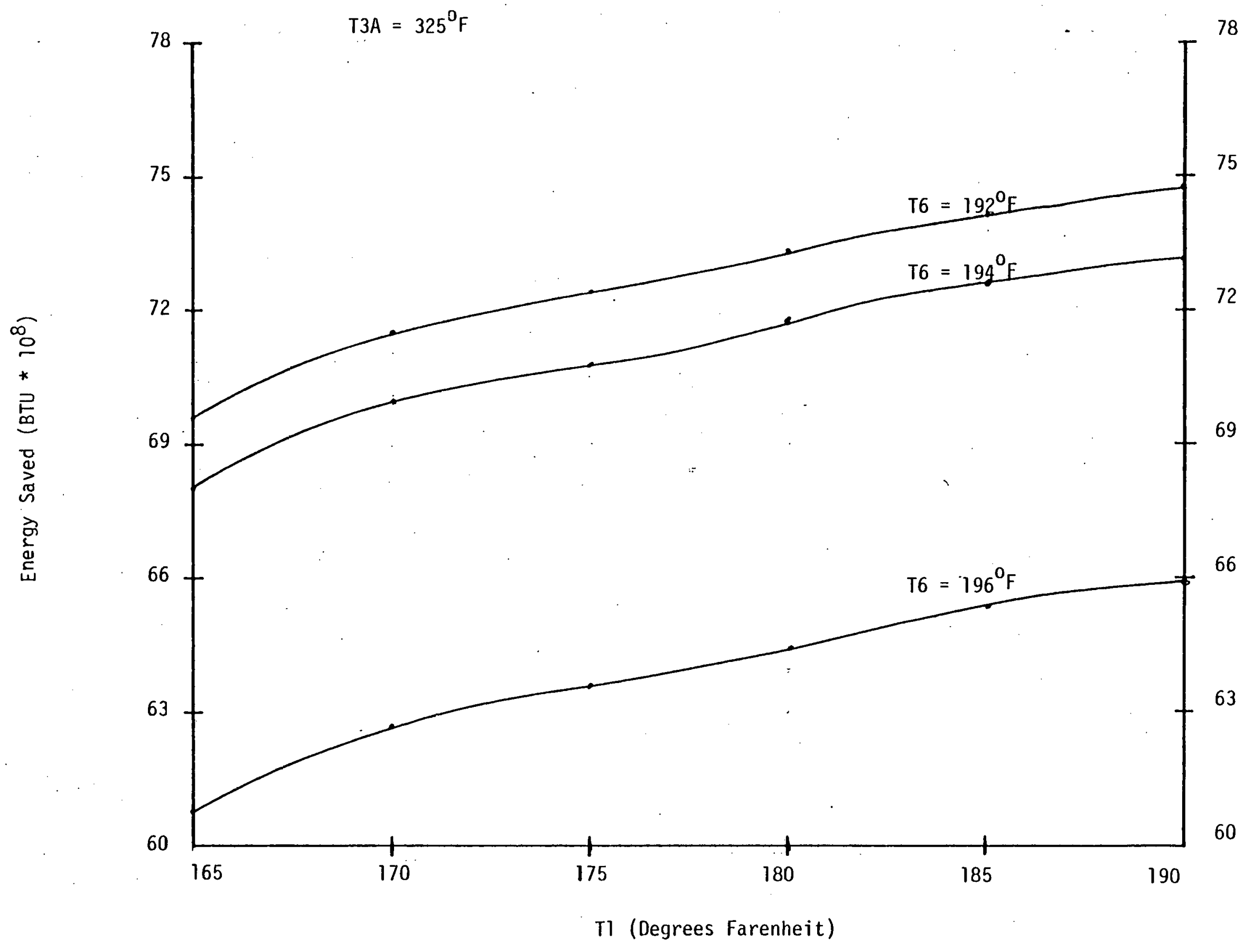


Figure 4.12. Heat Pump Performance Based on Energy

Saved For the Demonstration Model

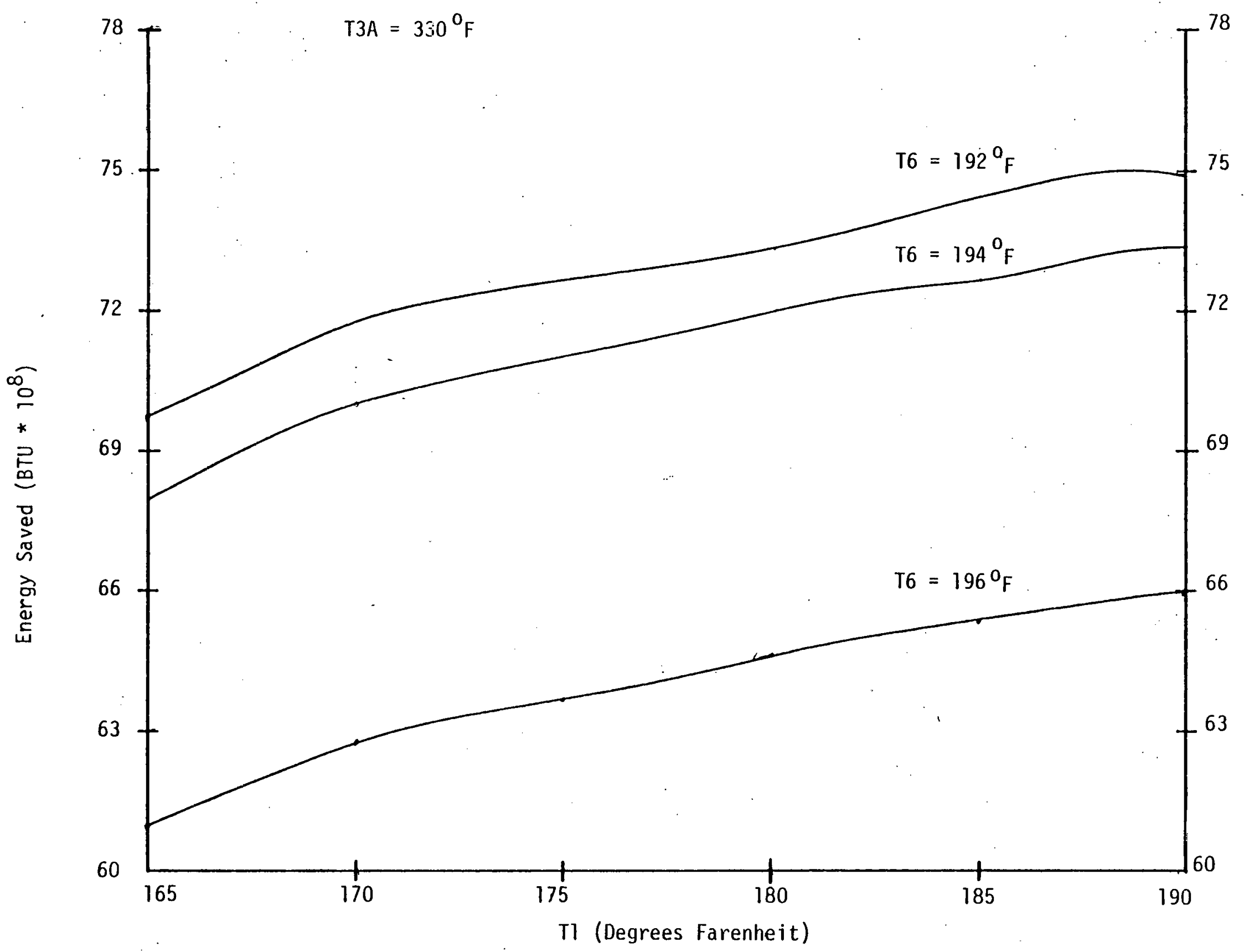


Figure 4.13. Heat Pump Performance Based on Energy Saved for the Demonstration Model

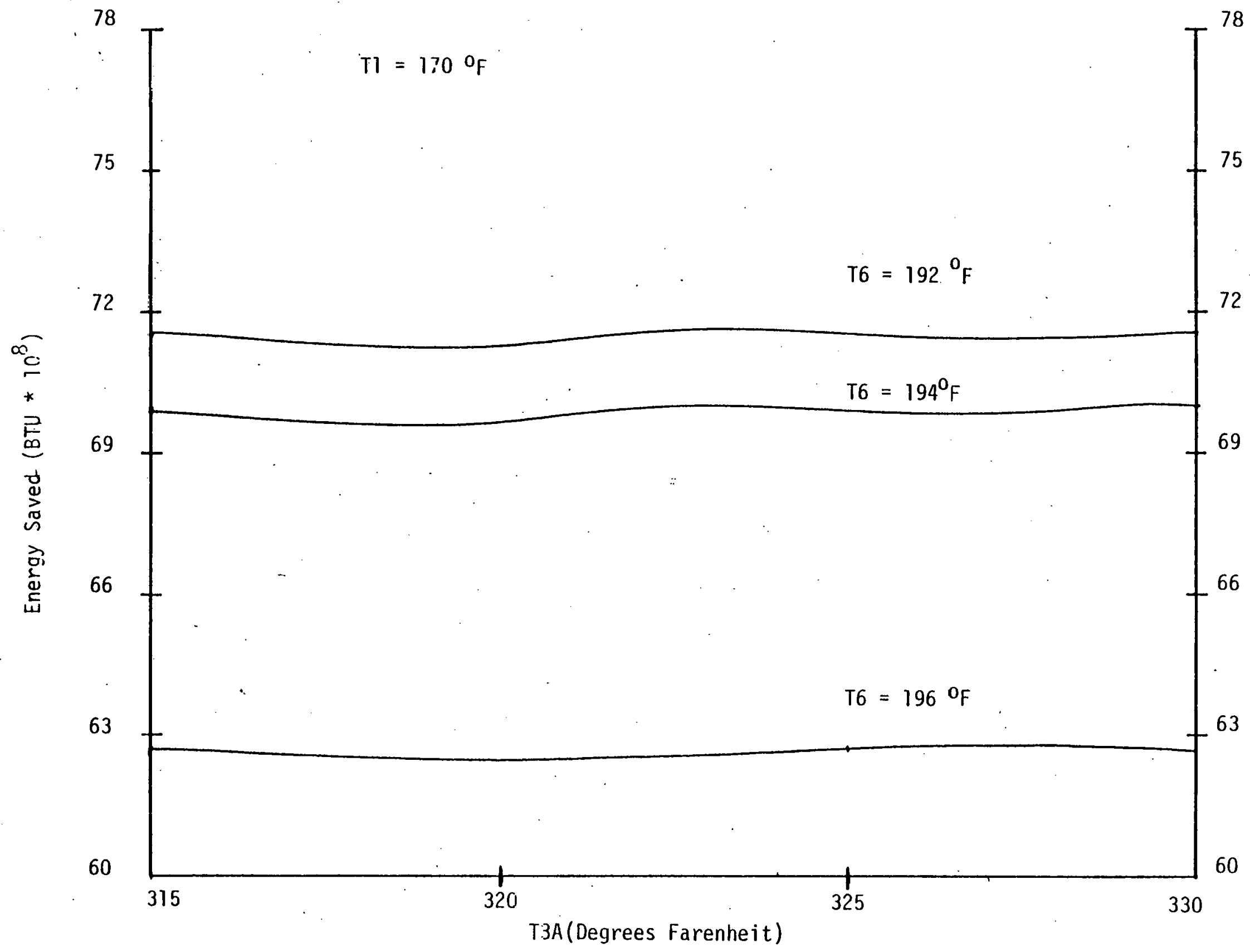


Figure 4.14. Heat Pump Performance Based on Energy

Saved for the Demonstration Model

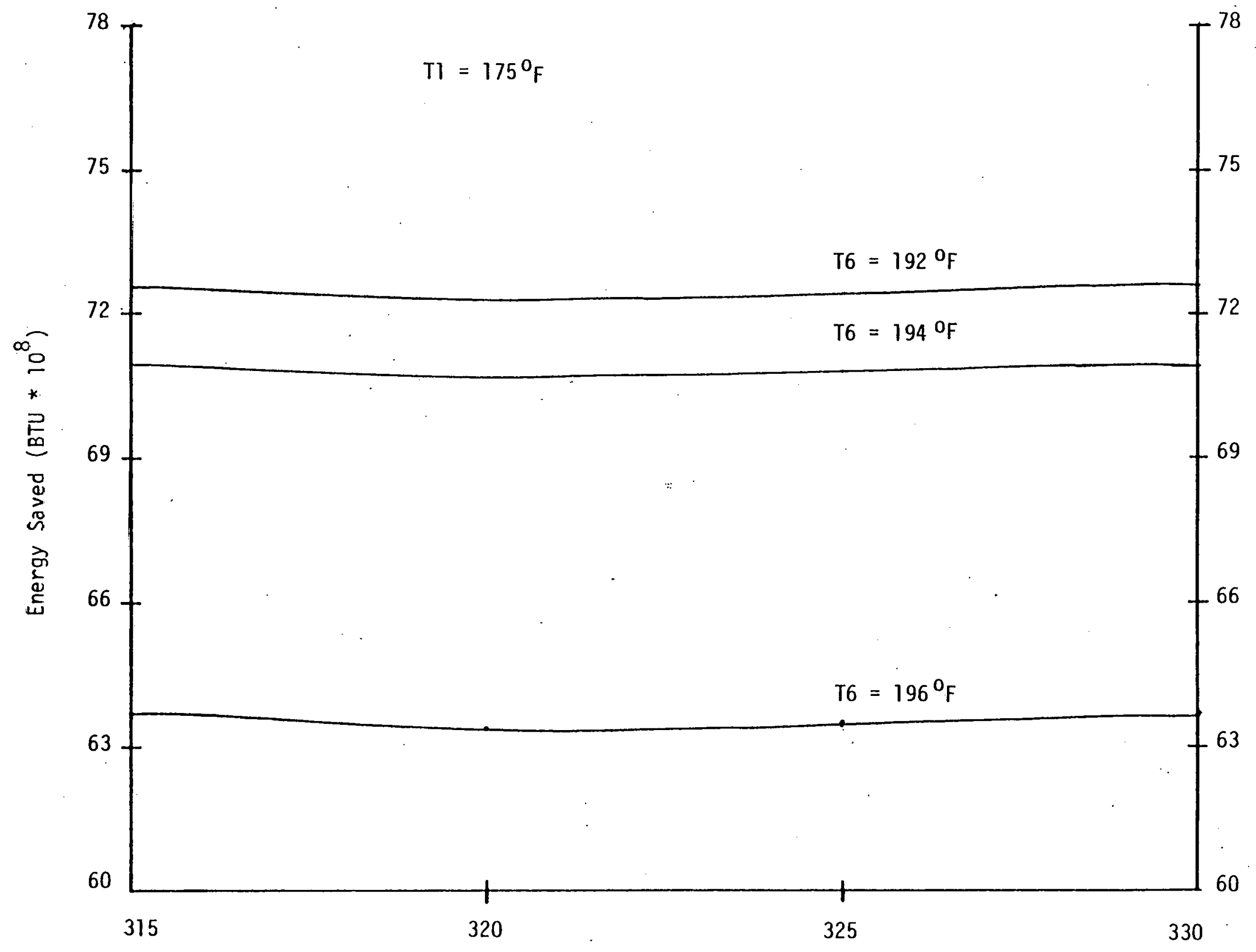

T3A (Degrees Farenheit). 
Figure 4.15. Heat Pump Performance Based on Energy Saved for the Demonstration Model

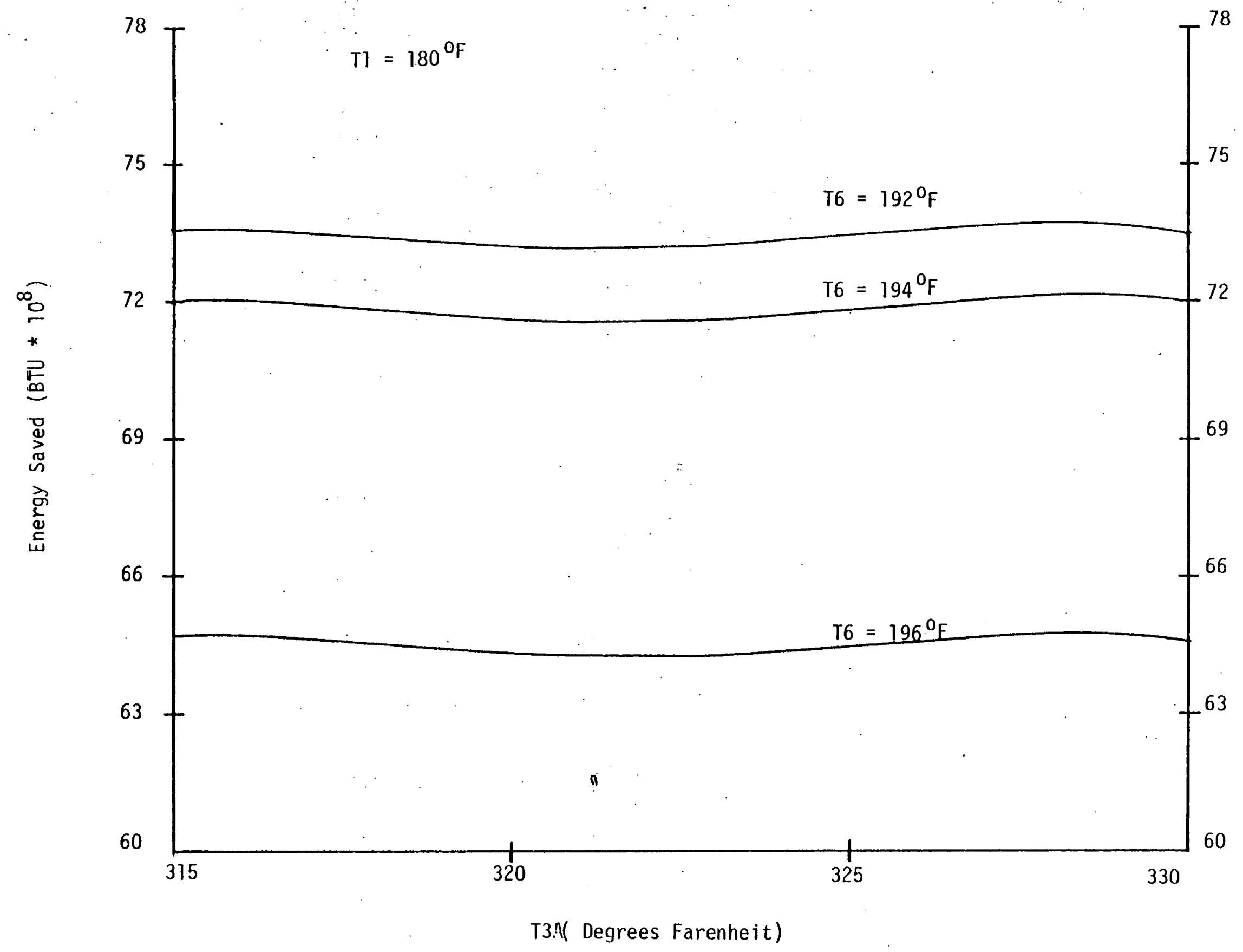


Figure 4.16. Heat Pump Performance Based on Return on Investment for an Industrial Unit

$\left(28,200 \quad \frac{1 \mathrm{bm} \text { of product }}{\text { hour }}\right)$

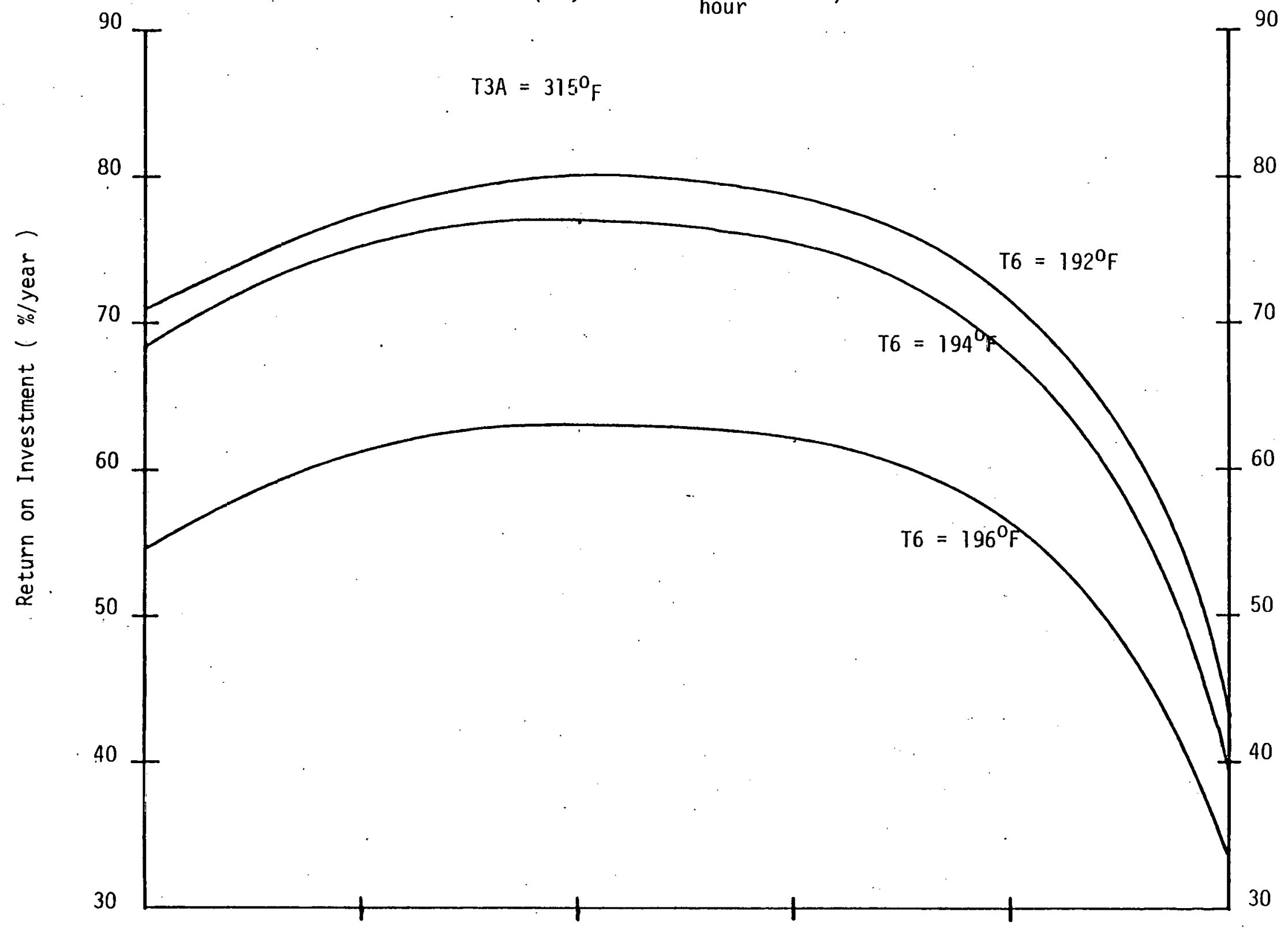

TI (Degrees Farenheit) 
Figure 4.17. Heat Pump Performance Based on Return on Investment for an Industrial Unit

$\left(2 \varepsilon, 200 \quad \frac{\mathrm{lbm} \text { of product }}{\text { hour }}\right)$

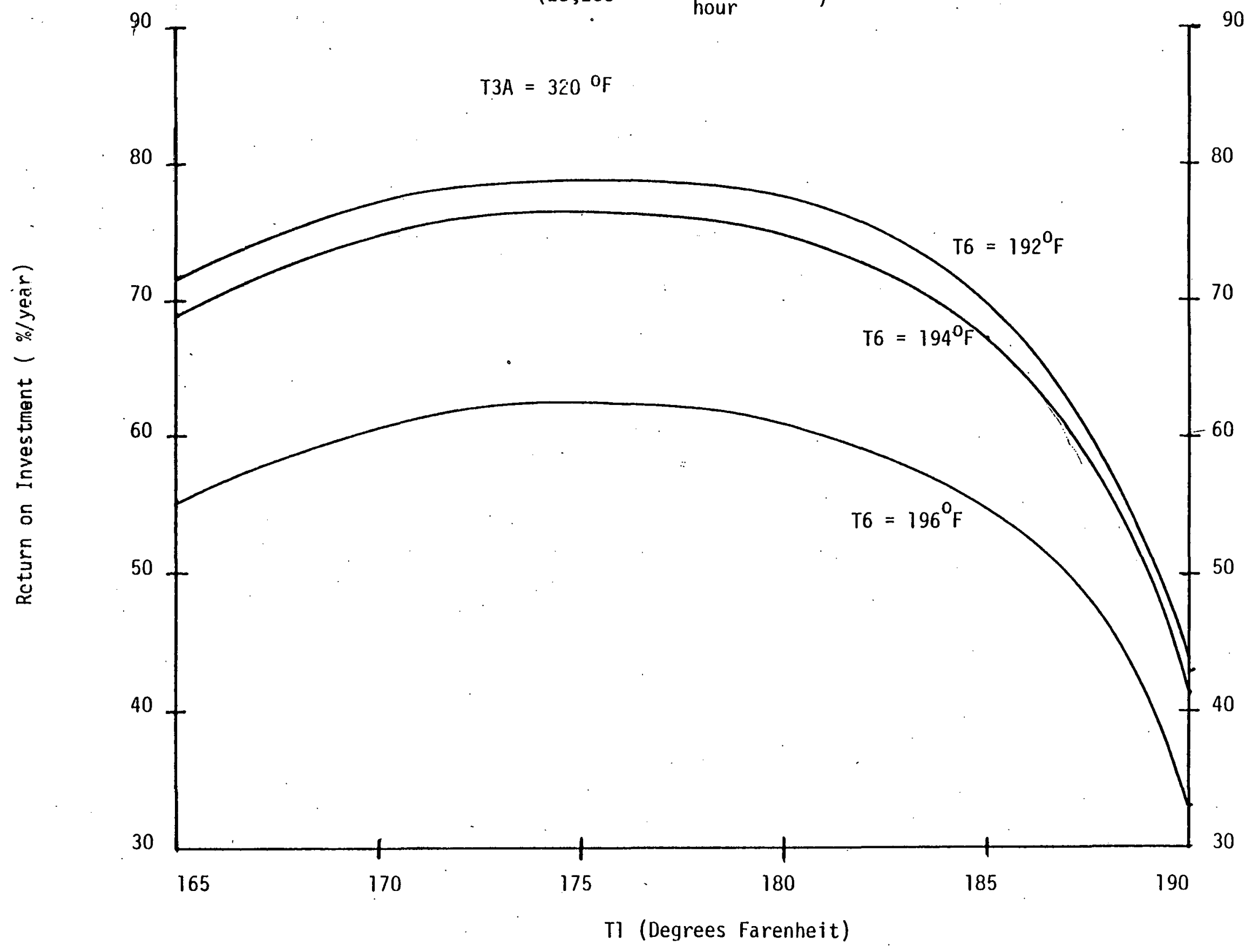


Figure 4.18. Heat Pump Performance Based on Return on Investment for an Industrial Unit

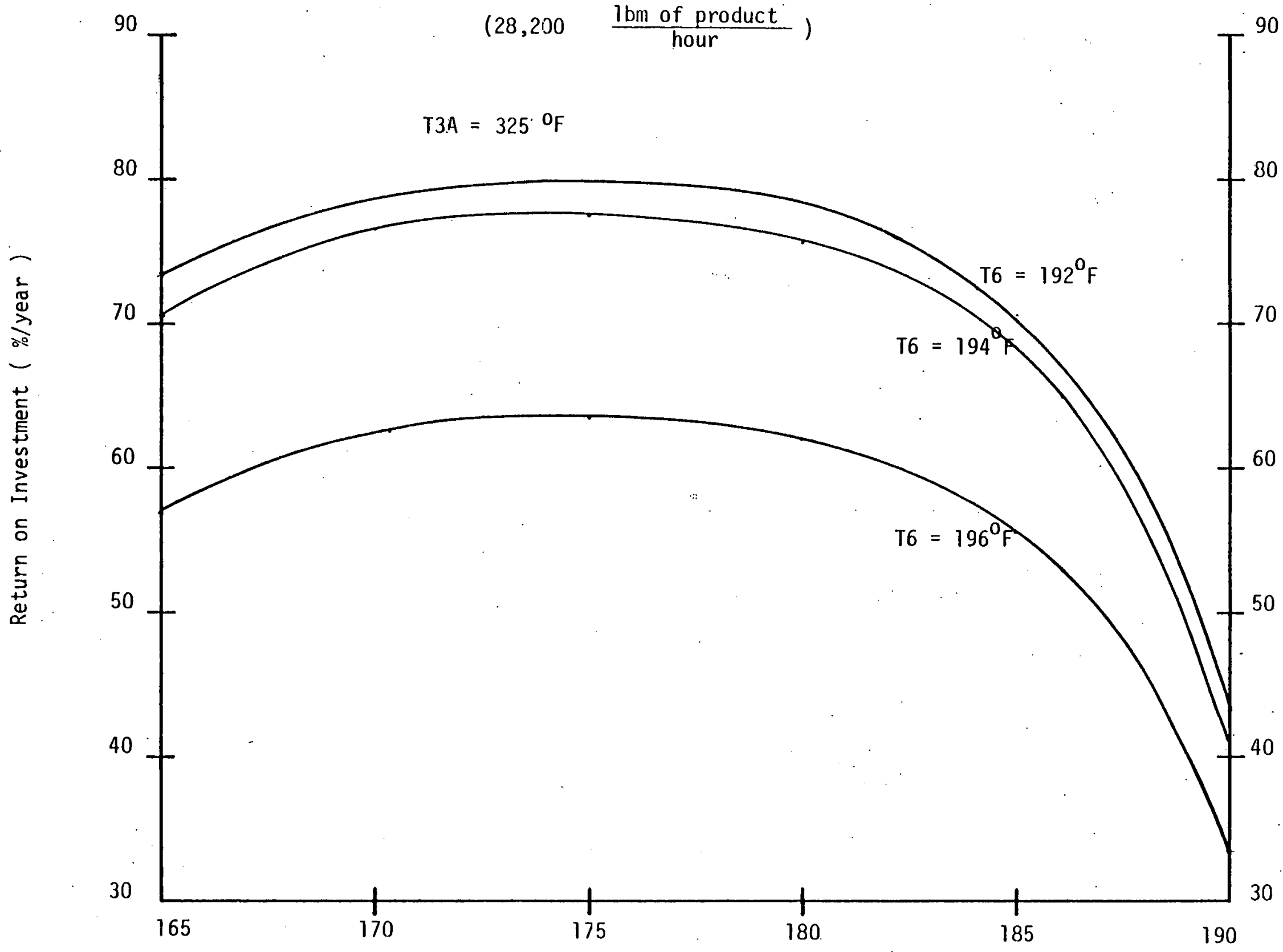

II (Degrees Farenheit) 
Figure 4.19. Heat Pump Performance Based on Return on Investment for an Industrial Unit

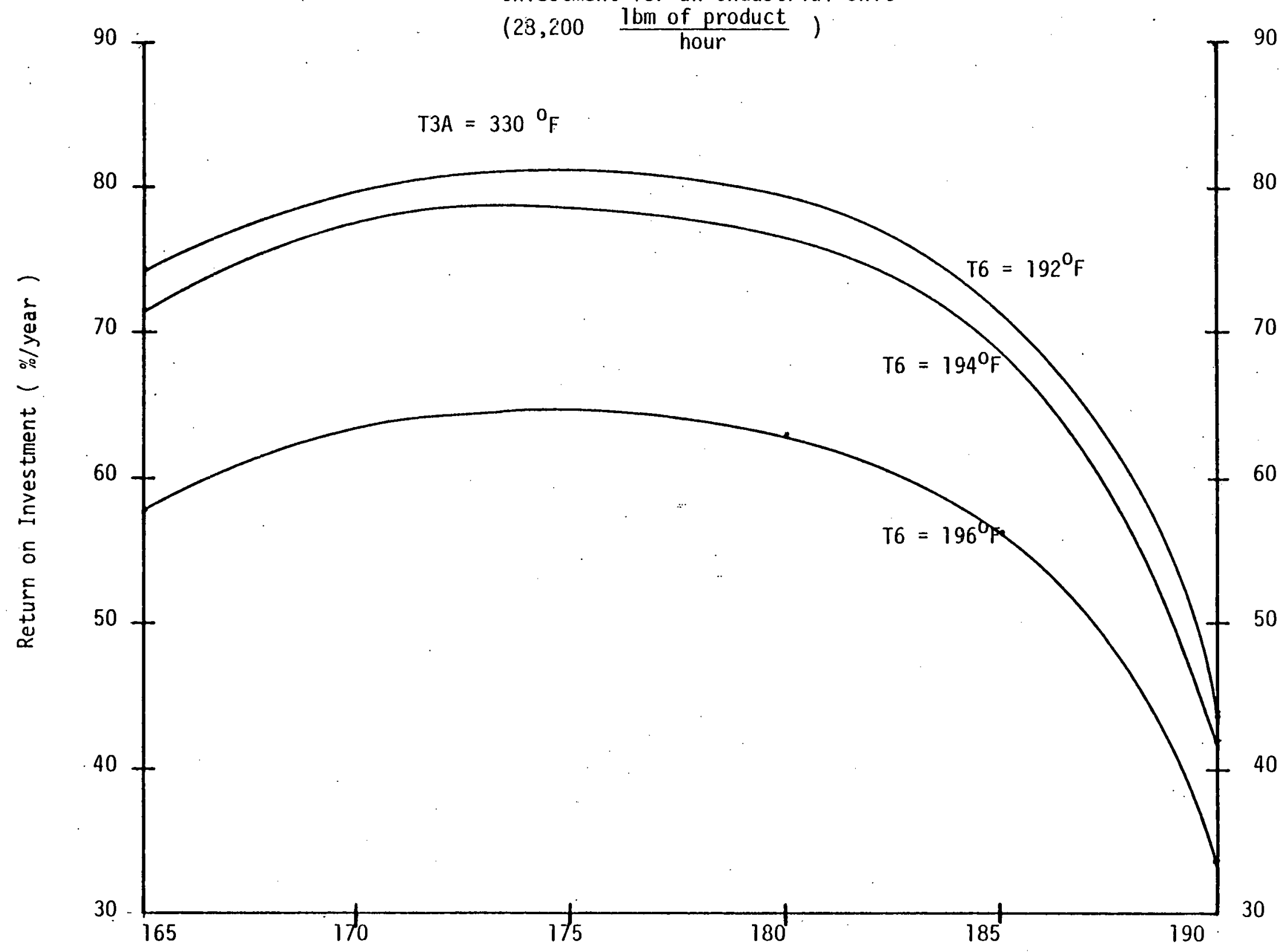


Figure 4.20. Heat Pump Performance Based on Return on Invesiment for an Industrial Unit

$\left(28,200 \frac{1 \mathrm{bm} \text { of product }}{\text { hour }}\right)$

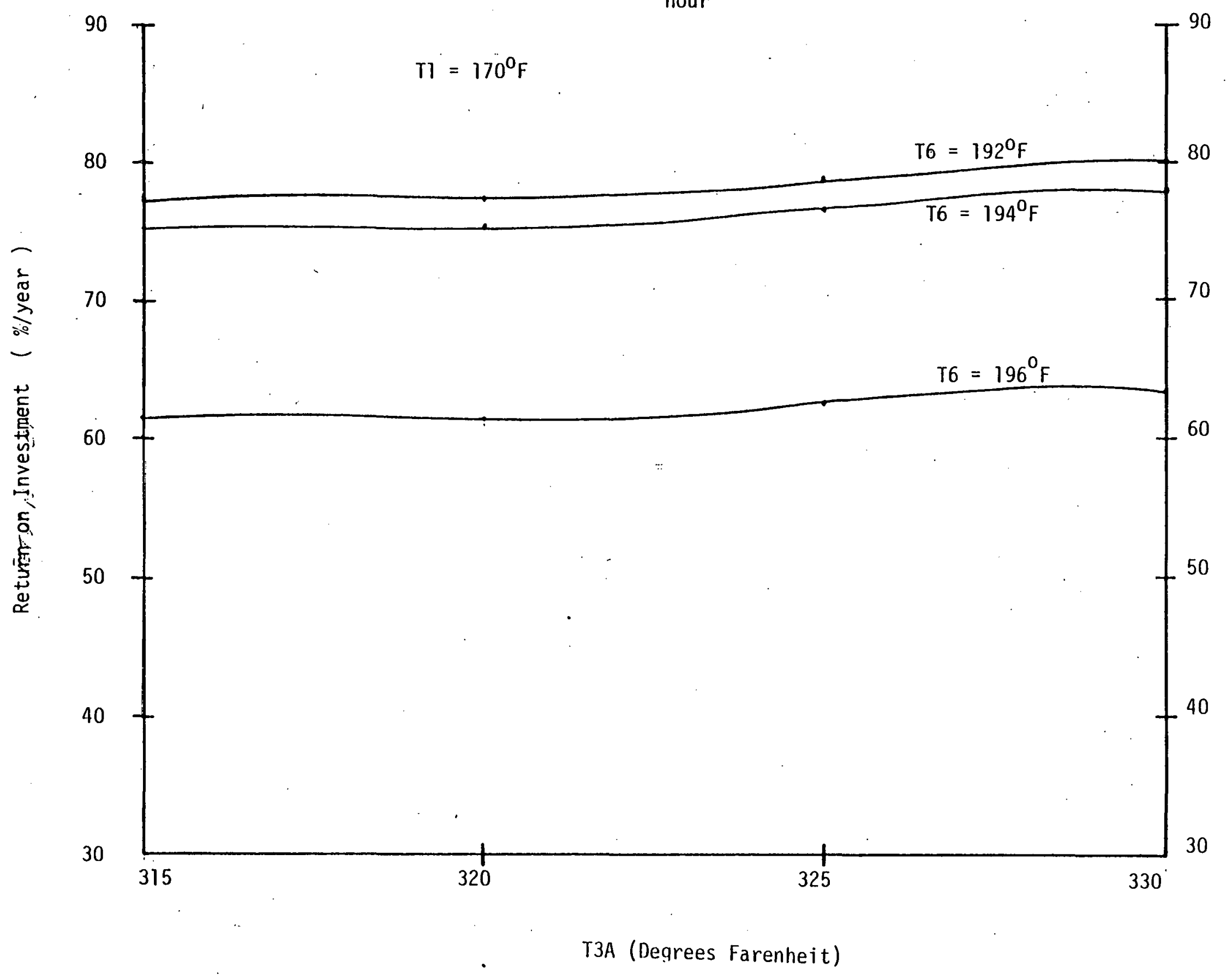




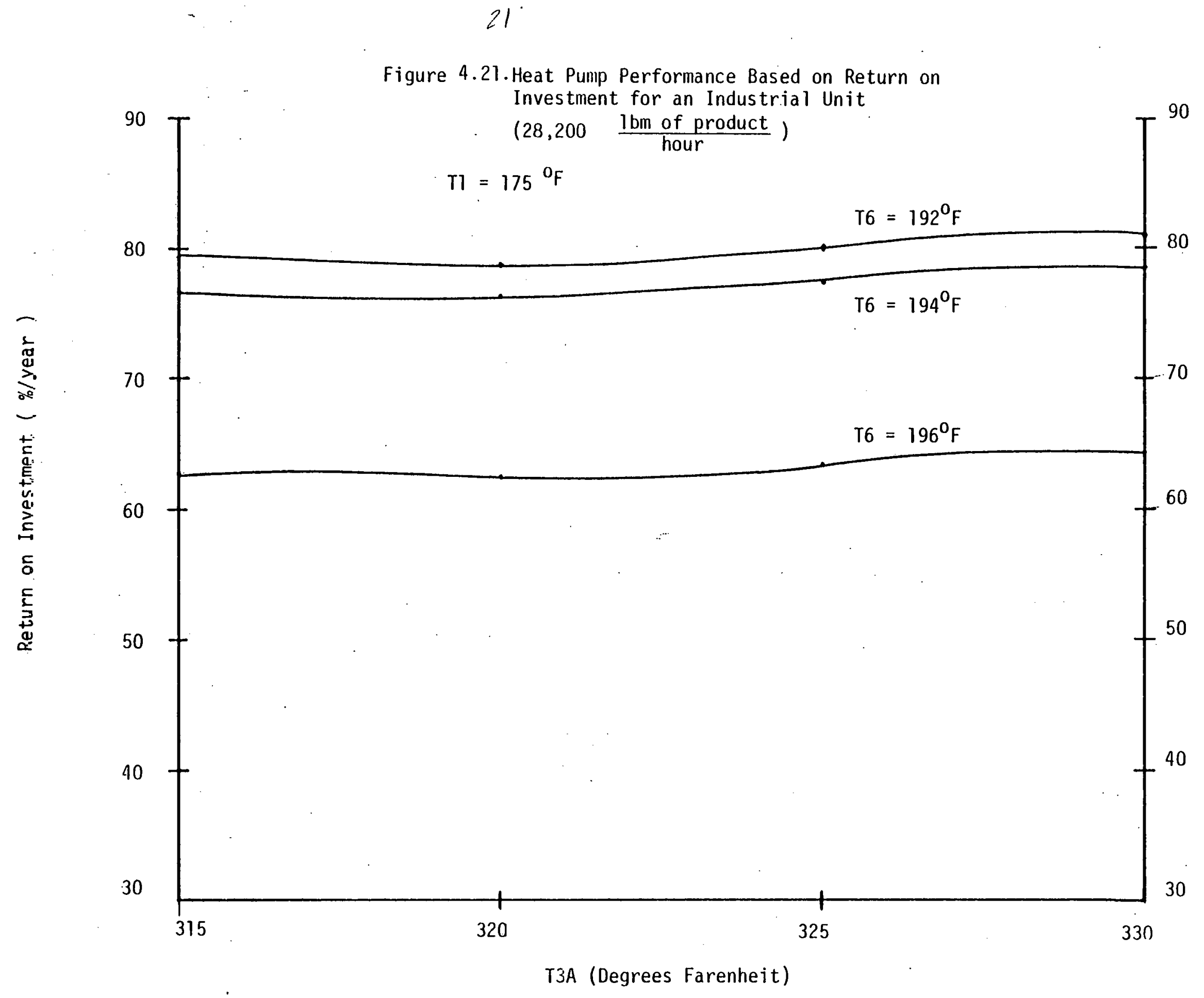


Figure 4.22. Heat Pump Performance Based on Return on Investment for an Industrial Unit

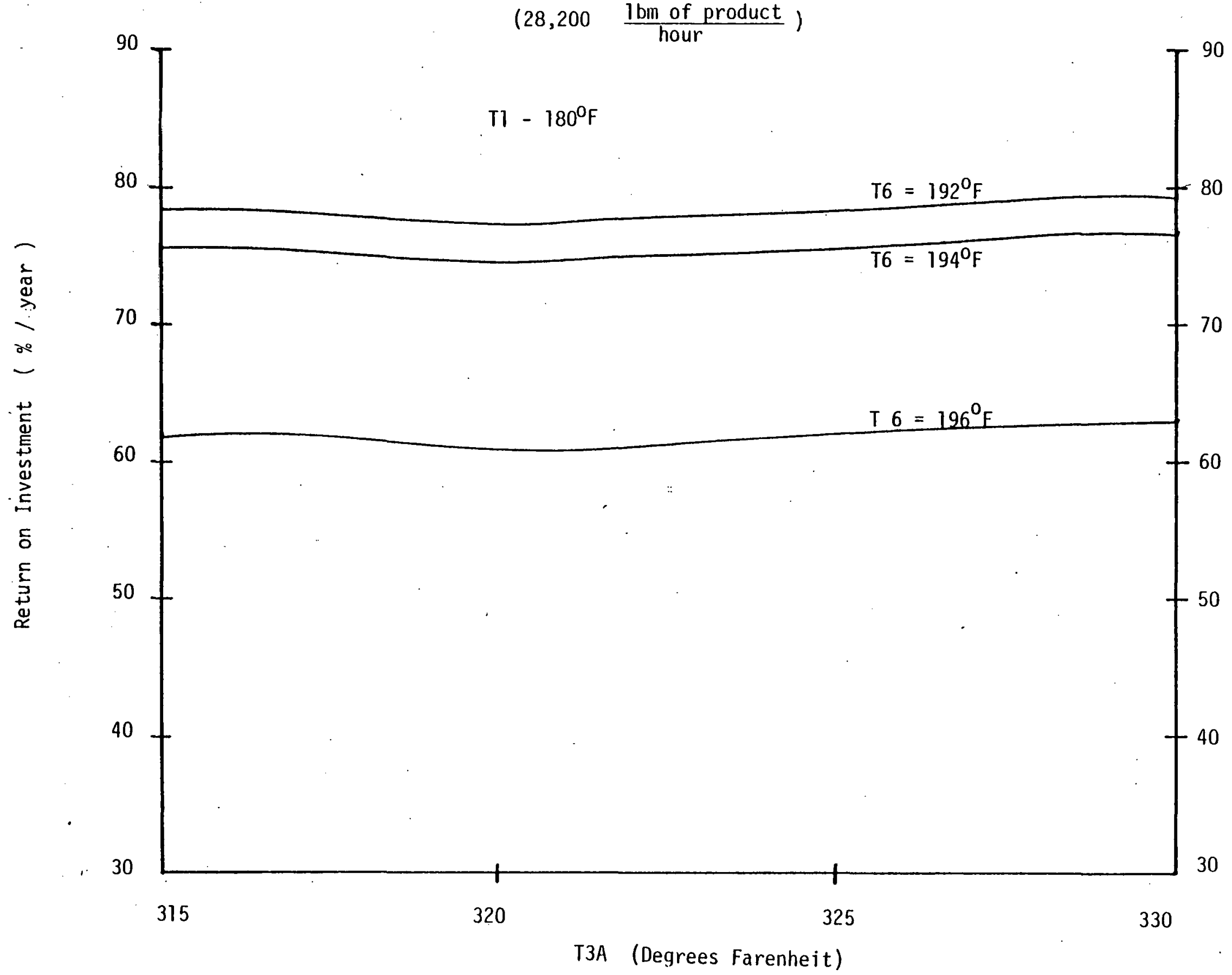


Fiqure 4.23. Hea: Pump Performance Based on Energy Saved For the Industrial Unit

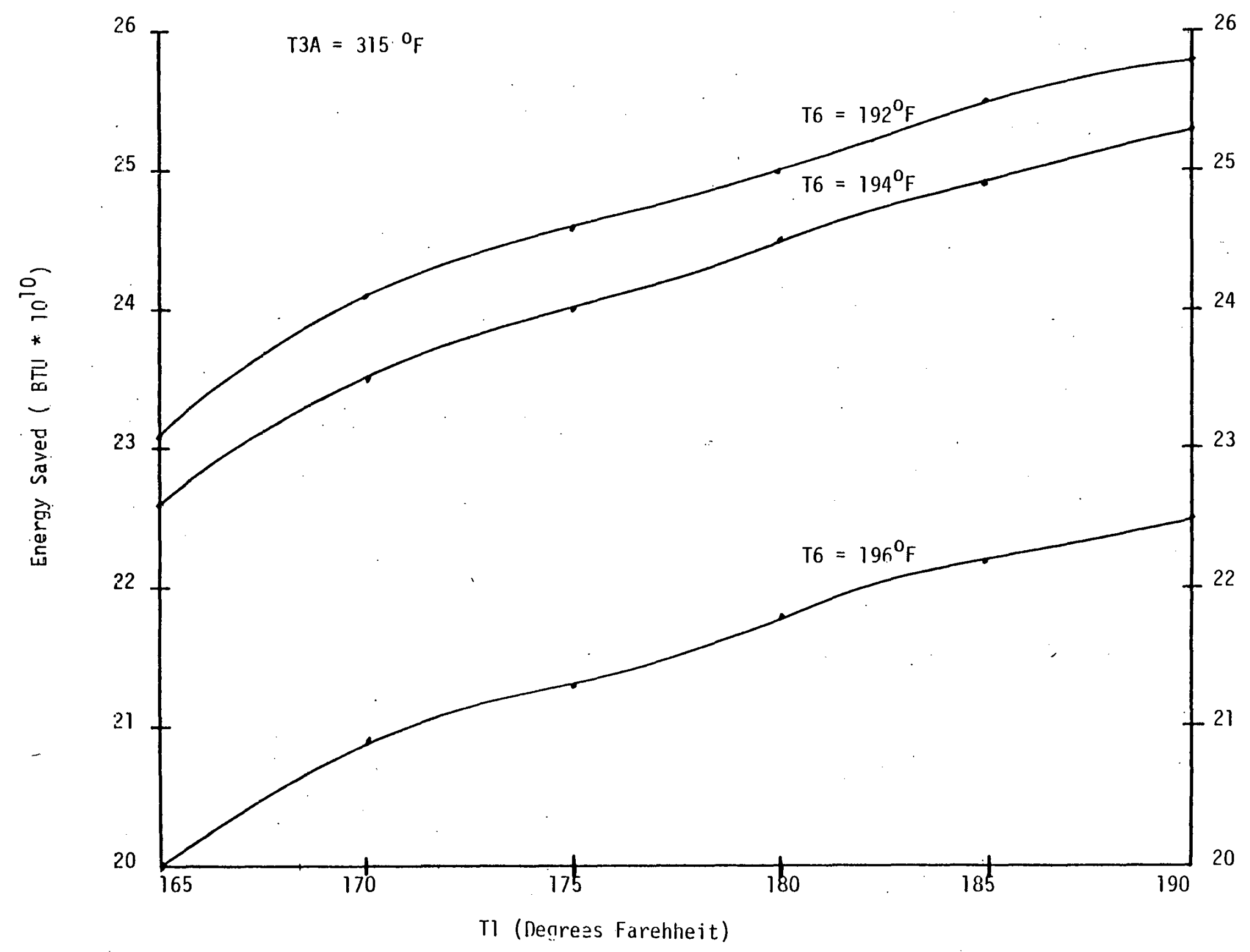


Figure 4.24. Heat Pump Performance Based on Energy Saved

For the Industrial Unit

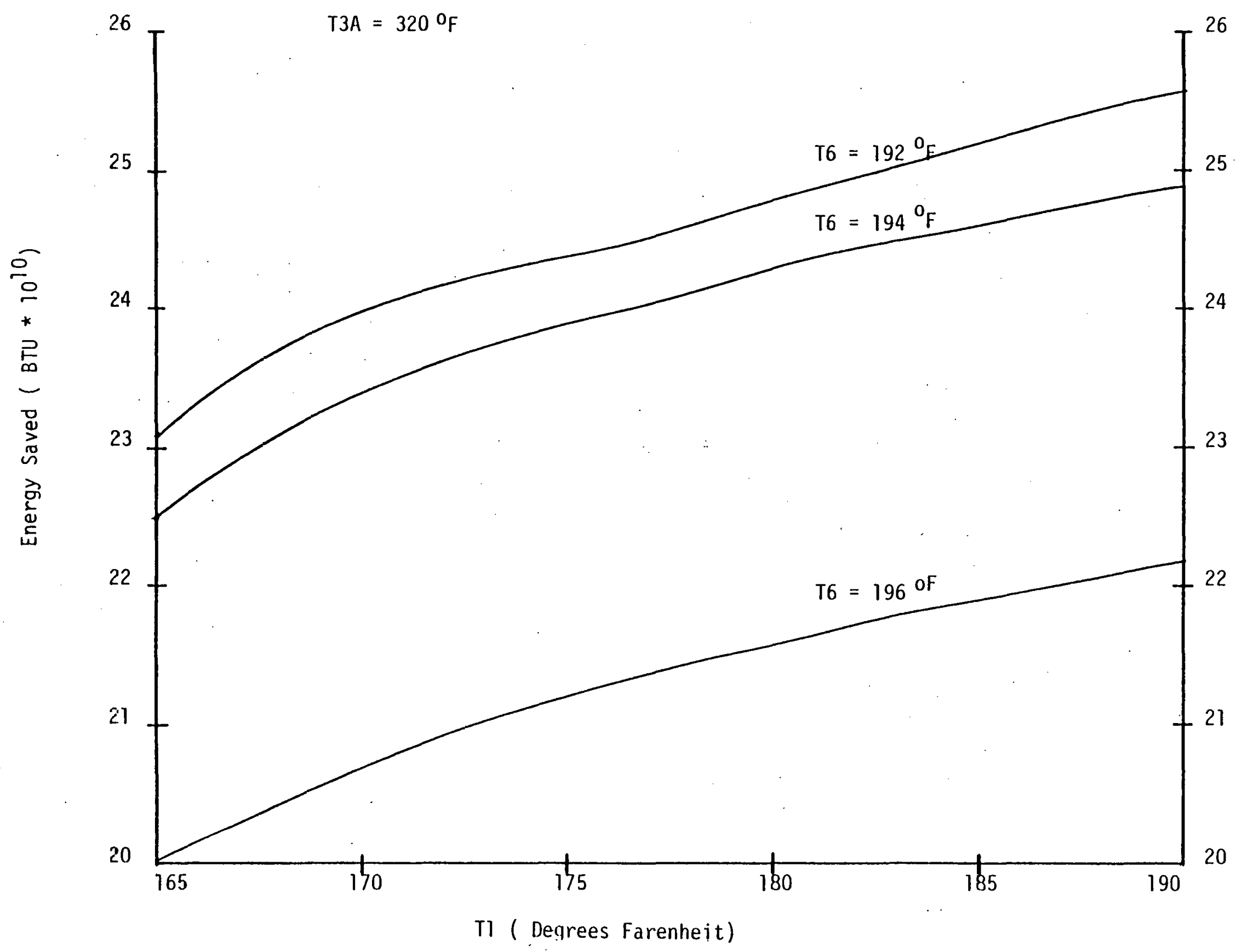


Figure 4.25. Heat Pump Performance Based on Energy Saved For the Industrial Unit

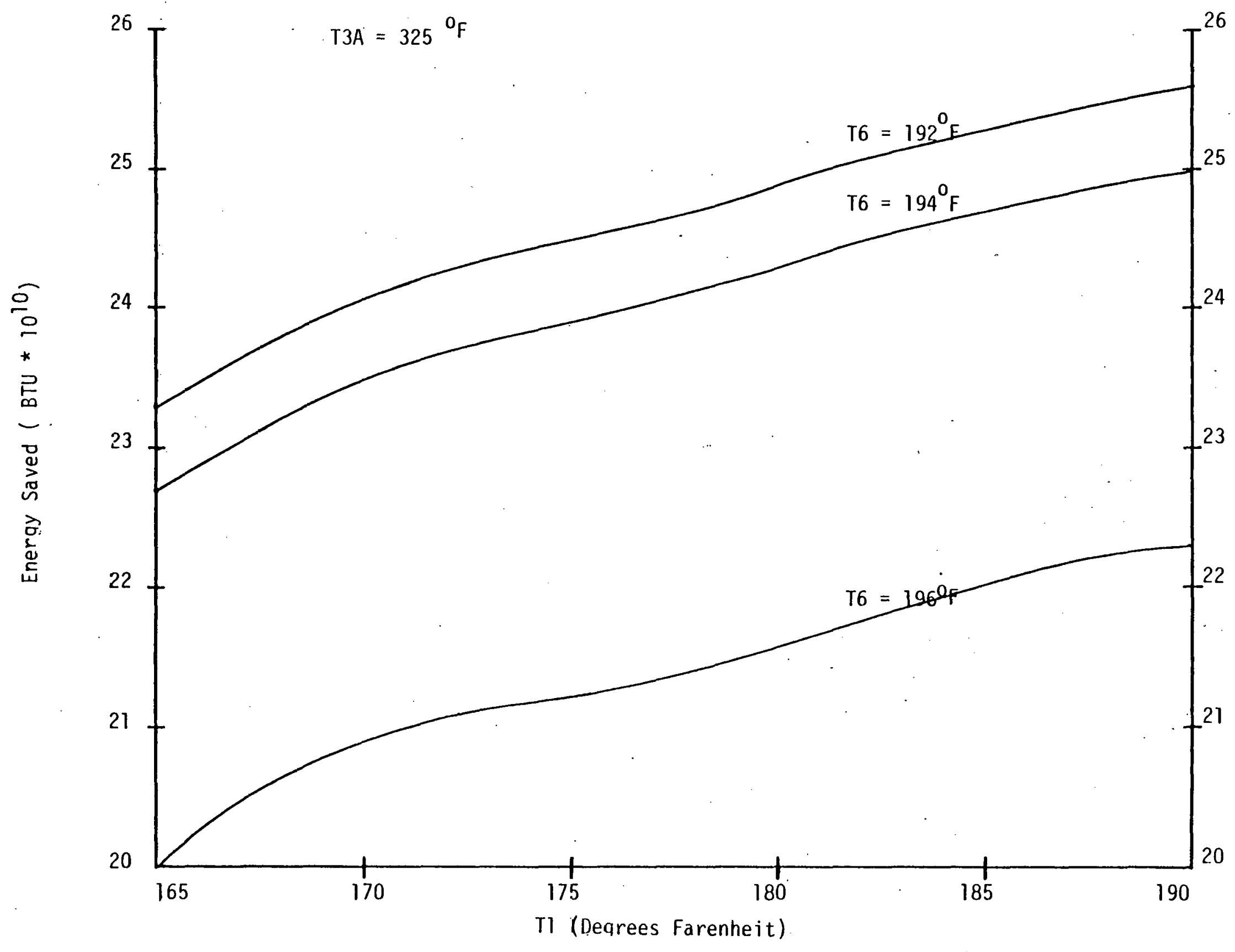


Figure 4.26. Heat Pump Performance Based on Energy Saved For the Industrial Unit

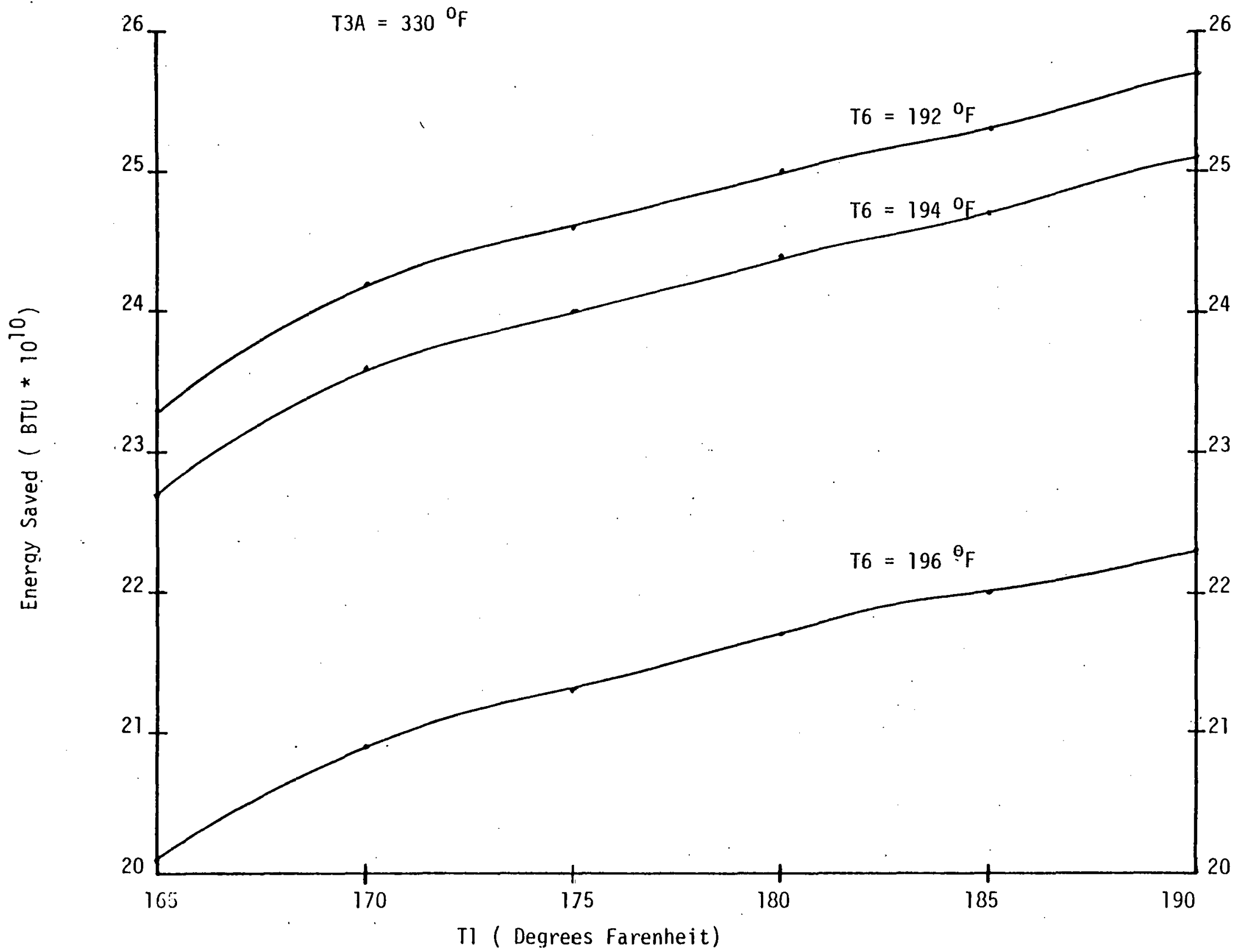


Figure 4.27. Heat Pump Performance Based on Energy Saved For the Industrial Unit

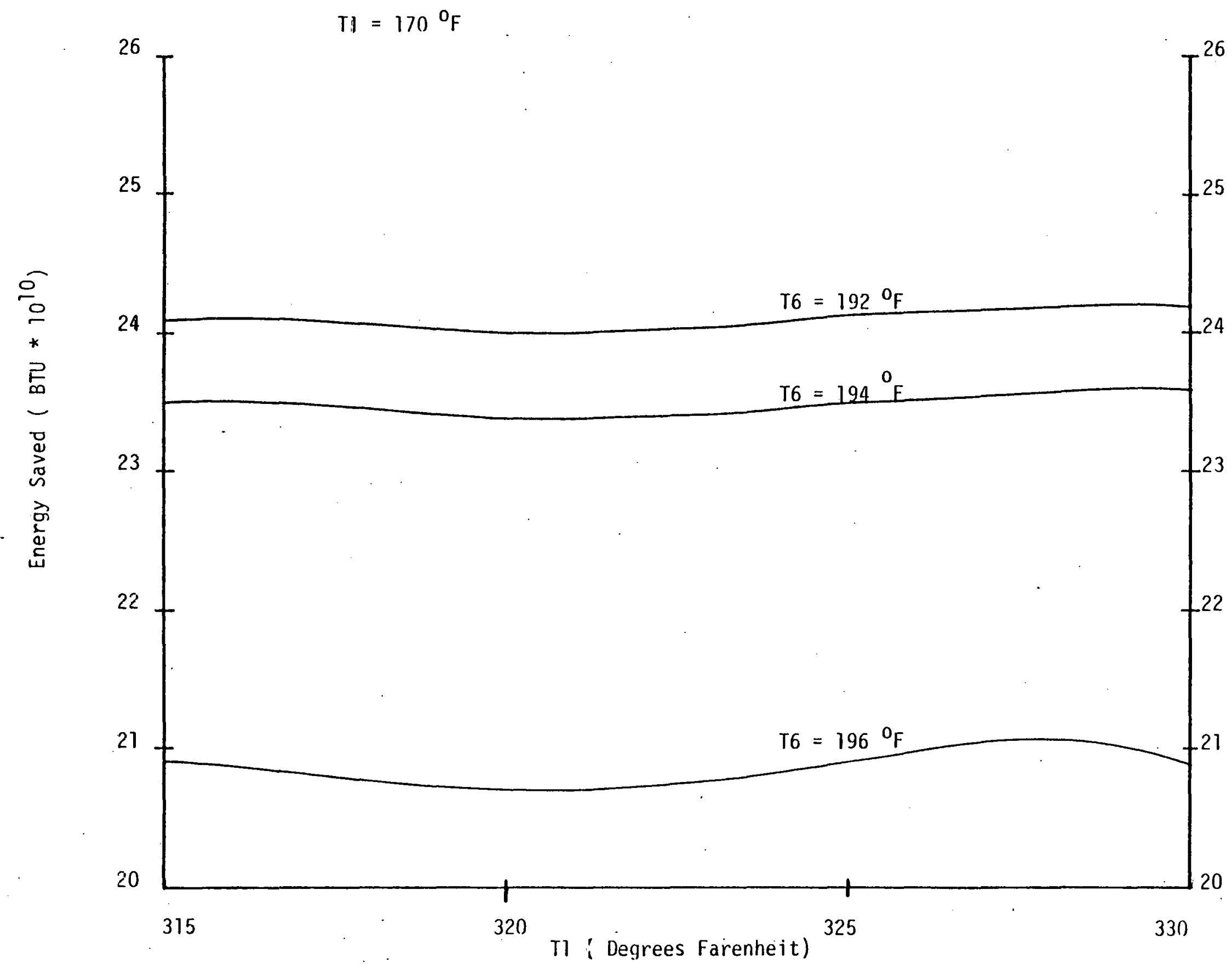


Figure 4.28. Heat Pump Performance Based on Energy Saved For the Industrial Unit

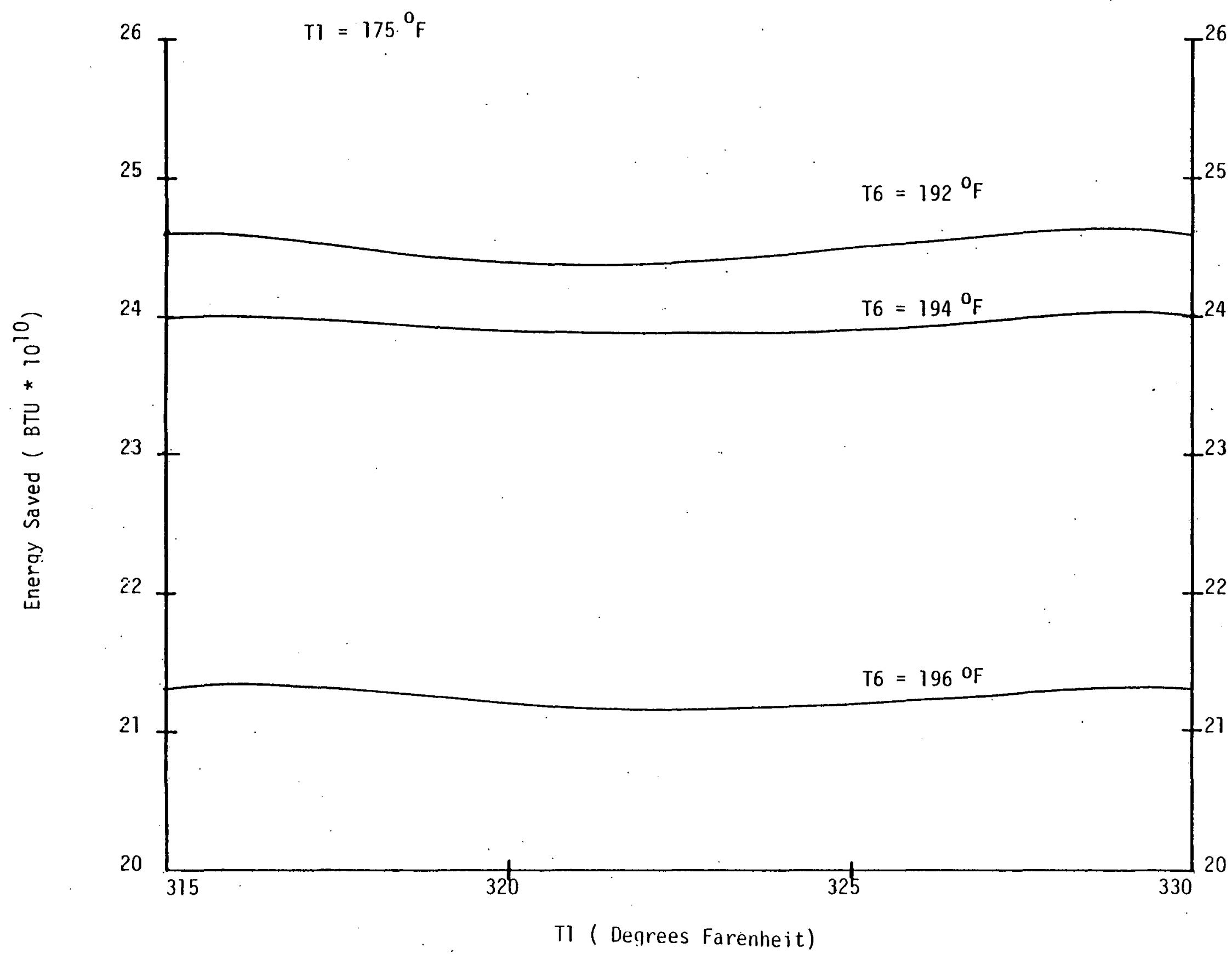


Figure 4.29. Heat Pump Performance Based on Energy Saved For the Industrial Unit

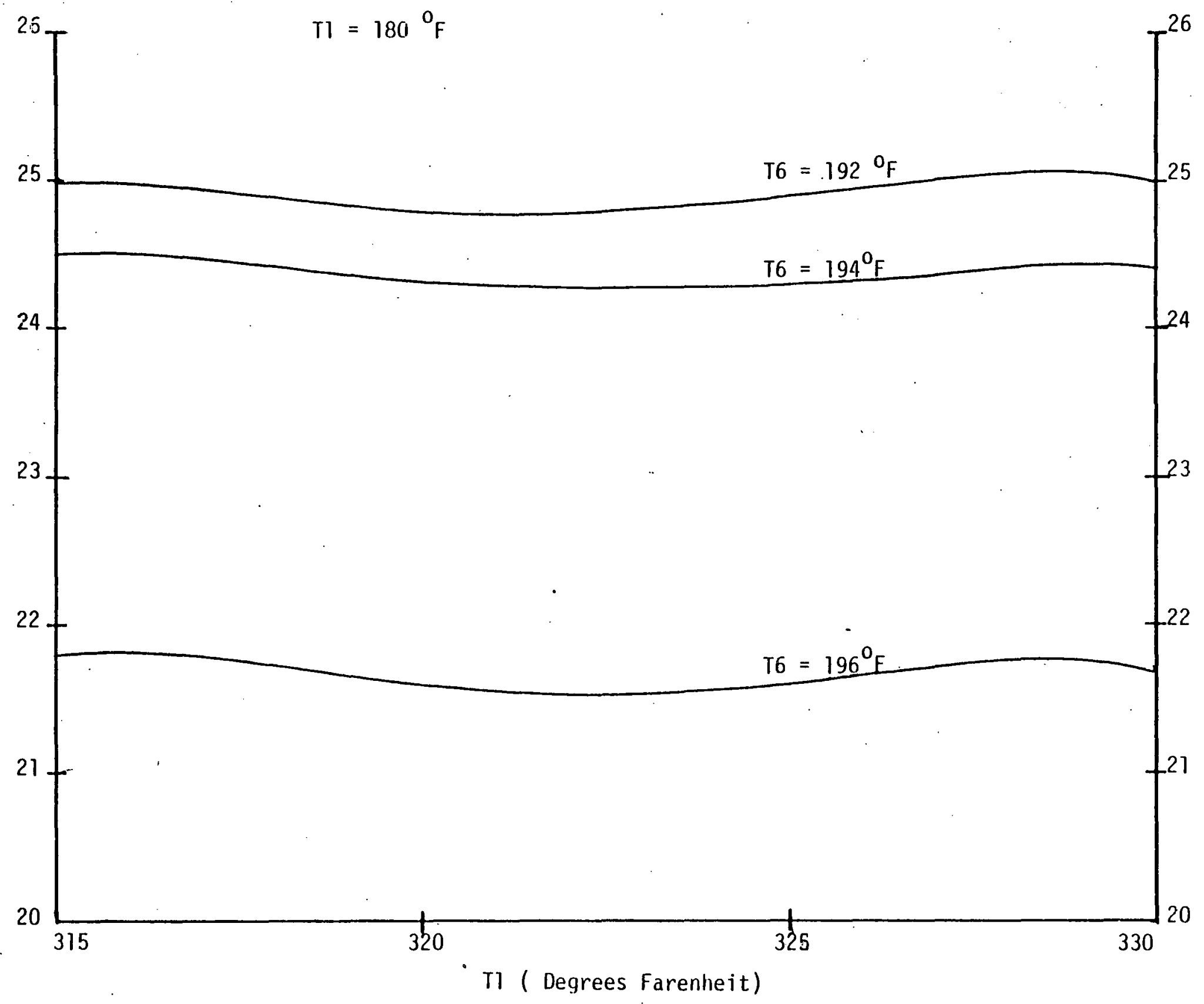


Chapter 5

SYSTEM COMPONENTS DESIGN

The heat pump waste heat recovery system includes ten component parts. These are: The compressor, the evaporator, the condensor, the air heater, the dryer, two accumulator tanks and three expansion valves. of these all but three, the dryer and two accumulator tanks, were quoted on by outside sources. Thus the first part of this chapter consists of materials sent to vendors by Auburn University and quotes received by Auburn University concerning equipment. The simulated dryer and two accumulator tanks were designed by Auburn University. The detailed design and pricing estimates are included. 


\section{The Compressor}

The compressor is the heart of the heat pump waste heat recovery system and represents the major expenditure of the demonstration unit construction. The demonstration compressor specified is a fifty horsepower two-stage unit with oil-free construction. The small size of this unit dictates the use of a reciprocating compressor instead of a centrifugal or screw type compressor that would normally be used in an industrial size system. The high compressor exit temperature and oil-free construction are requirements that are not well. met by this type compressor and thus require specialized engineering and construction. This fact is reflected in the unusually high cost (dollars per horsepower) of this compressor. However, the stated requirements pose no major problems for larger centrifugal: and screw type compressors. The following pages contain the specifications sent to Ingersoll-Rand and the quotation recefved by Auburn University. At the time of this writing, Ingersoll-Rand was the only manufacturer located with the ab1l1ty tu produce this compressor. 


\section{3}

The compressor is a two stage reciprocating type with unaqual flow rates. The working fluid is methanol which is a dry compressing fluid. Saturated methanol vapor at 25.5 PSIA (STATE 2) enters the first stage of compression and exits as a superheated vapor (STATE ISH). This vapor is de-superheated by bubling it through a saturated liouid at the same pressure. During this process part of the liquid is evaporated increasing the mass flow rate at STATE ISAT. The saturated vapor at STATE ISAT enters the second stage of compression and exits as a superheated vapor (STATE 3). Methanol has shown highiy corrosive behavior in the presence of aluminum at temperatures above $300^{\circ} \mathrm{F}$ therefore no aluminum parts should be used. Since methanol is a liquid at room temperature the compressor should have heaters installed for start-up protection.

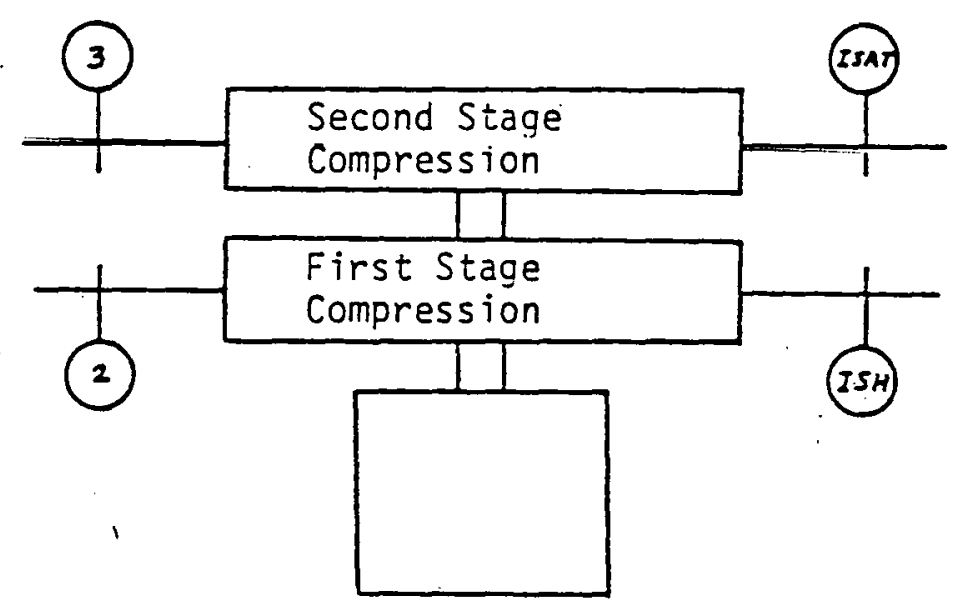


5.4

\section{STATE 2}

$T 2=175^{\circ} \mathrm{F}$

$P 2=25.6$ PSIA

$\mathrm{h} 2=549 \mathrm{BTU} / \mathrm{lbm}$

$\dot{M} 2=1232.1 \mathrm{lbm} / \mathrm{Hr}$

$\dot{V}_{2}=9762.8 \mathrm{Ft}^{3} / \mathrm{Hr}$

SATURATED VAPOR.

STATE ISAT

TISAT $=248.9^{\circ} \mathrm{F}$

PISAT $=85.3$ PSIA

hISAT $=559.5 \mathrm{BTU} / 1 \mathrm{bm}$

MISAT $=1358 \mathrm{1bm} / \mathrm{Hr}$

VISAT $=2893.7 \mathrm{Ft}^{3} / \mathrm{Hr}$

SATURATED VAPOR
STATE ISH

TISH $=305^{\circ} \mathrm{F}$

PISH $=85.3$ PSIA

$h I S H=601.9 \mathrm{BTU} / 1 \mathrm{lbm}$

$\dot{M} I S H=1232.1 \mathrm{lbm} / \mathrm{Hr}$

$\dot{V}$ ISH $=3725 \mathrm{Ft}^{3} / \mathrm{Hr}$

SUPERHEATED VAPOR
$T 3=388^{\circ} \mathrm{F}$

$P 3=284$ PSIA

$h 3=605.2 \mathrm{BTU} / 1 \mathrm{bm}$

$\dot{M} 3=1358 \mathrm{ibm} / \mathrm{Hr}$

$\dot{v}_{3}=2379.6 \mathrm{Ft}^{3} / \mathrm{Hr}$

SUPERHEATED VAPOR 
PROPERTIES OF METHANOL

Molecular Weight

Boiling Point (normal)

Melting Point

Critical Temperature

Critical Pressure

Critical Specific Volume

Critical Compressibility

Constant Pressure Specific Heat
32.04

$148.46^{\circ} \mathrm{F}$

$-143.68^{\circ} \mathrm{F}$

$462.92^{\circ} \mathrm{F}$

1174.3 psia

$.0583 \mathrm{it}^{3} / \mathrm{Tbm}$

.224

Using $\quad c_{v}=C_{p}-R$

$$
\begin{aligned}
& c_{v}=c_{p}-\frac{1.097}{32.04} \\
& k=\frac{c_{p}}{c_{v}} \\
& k=\frac{3.61 \times 10^{-4} \times T+.298}{3.61 \times 10^{-4} \times T+.298-\frac{1.097}{32.04}} \\
& k=\frac{3.61 \times 10^{-4} \times T+.298}{3.61 \times 10^{-4} \times T+.2638}
\end{aligned}
$$

Thus

$$
\begin{array}{rlr}
T & =175^{\circ} \mathrm{F} & 1.105 \\
T & =212^{\circ} \mathrm{F} & 1.100 \\
T & =350^{\circ} \mathrm{F} & 1.088 \\
T & =392^{\circ} \mathrm{F} & 1.084
\end{array}
$$




\title{
5.6
}

\section{Reciproceting Compressor EST IMAT ING \\ Proposel \\ TO}

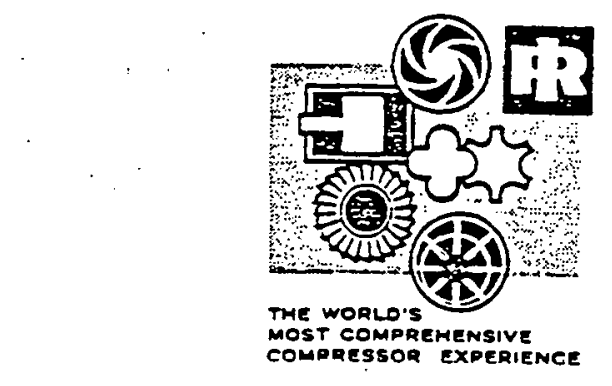

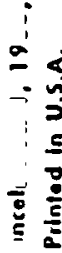

0
$=0$
0
0
0
$=\frac{0}{\overline{0}}$
$=\frac{0}{0}$
0
0
0
0

\author{
AUBURN UNIVERSITY \\ DOE PROJECT \\ HIGH TEMPERATURE METHANOL COMPRESSOR
}

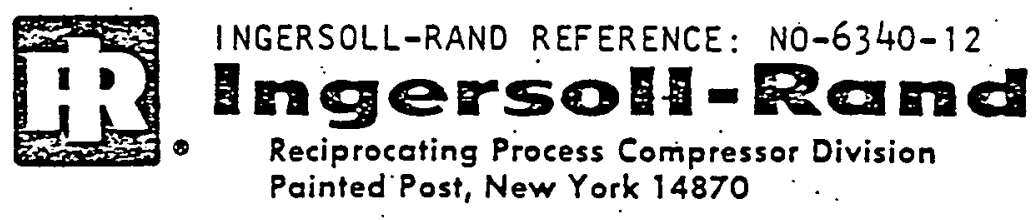

The world's most comprehensive compressor experience

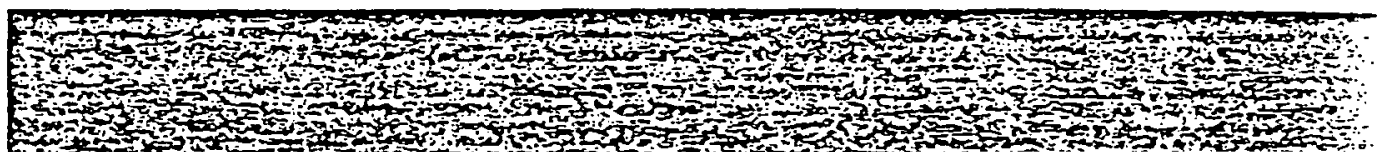




\section{Estimating

Purchaser Ref. DOE PROJECT

To AUBURN UNIVERSITY

Job no.

Ingersoll
ONE ONE (1) 11 \& $6 \times 9$ ESH - 2
Item no.

(hereinafter called Purchaser).

proposal.

EACH with equipment as described bolow.

\section{Standard Equipment}

- Compressor treme with erosshood oxtensions, eronkshot, bearlags, connecting rods, and erossheads.

- Compressor cylinders, plstons, rods, valves and poeking.

- Standard frame Iubrication system with lube oll pump, fher, cooler. gouge, shutdown switch and complete oll piping.

pressure seuee, erenkease oil level

- Force-feed lubrication of compressor cylinders with pre-fired tubing.

- Rolled threads on hardened carbon steol piston rods.

- Single-compartment distance piece.

- Suitoble driver cennectlon.

- Barring dovies; special wronches and tools.

- Foundation and general arringement plans, complete instruction books and part lists.

\section{Performance}

Service \& Slage

"K" value

Molocular weight

$\Delta$ Purehasoe's spocified eopacity

Inlel pressure, PSIA

Inlet temparature, ${ }^{\circ} \mathrm{F}$

Discharge pressure, PSIA

Oitcharge iemperature (theoretical), ${ }^{\circ}$

Compressibility at inlet (zi) (1)

Comprassibility of disch. (Zd) (1)

$R$ olativa $h \cdot: \pi i d i^{2} y(\%:)$

No. \& sixe eylinder:

RPM

Piston displacement, CFM

finles CFM (gueranteed)

$\Delta$ Compressor design capocily

BHP per service

†BHP - total (guerantead)

Number capocity control steps

Minimum capacity, \% design

Cylinder metorial

Cylinder flange size

Remorks:

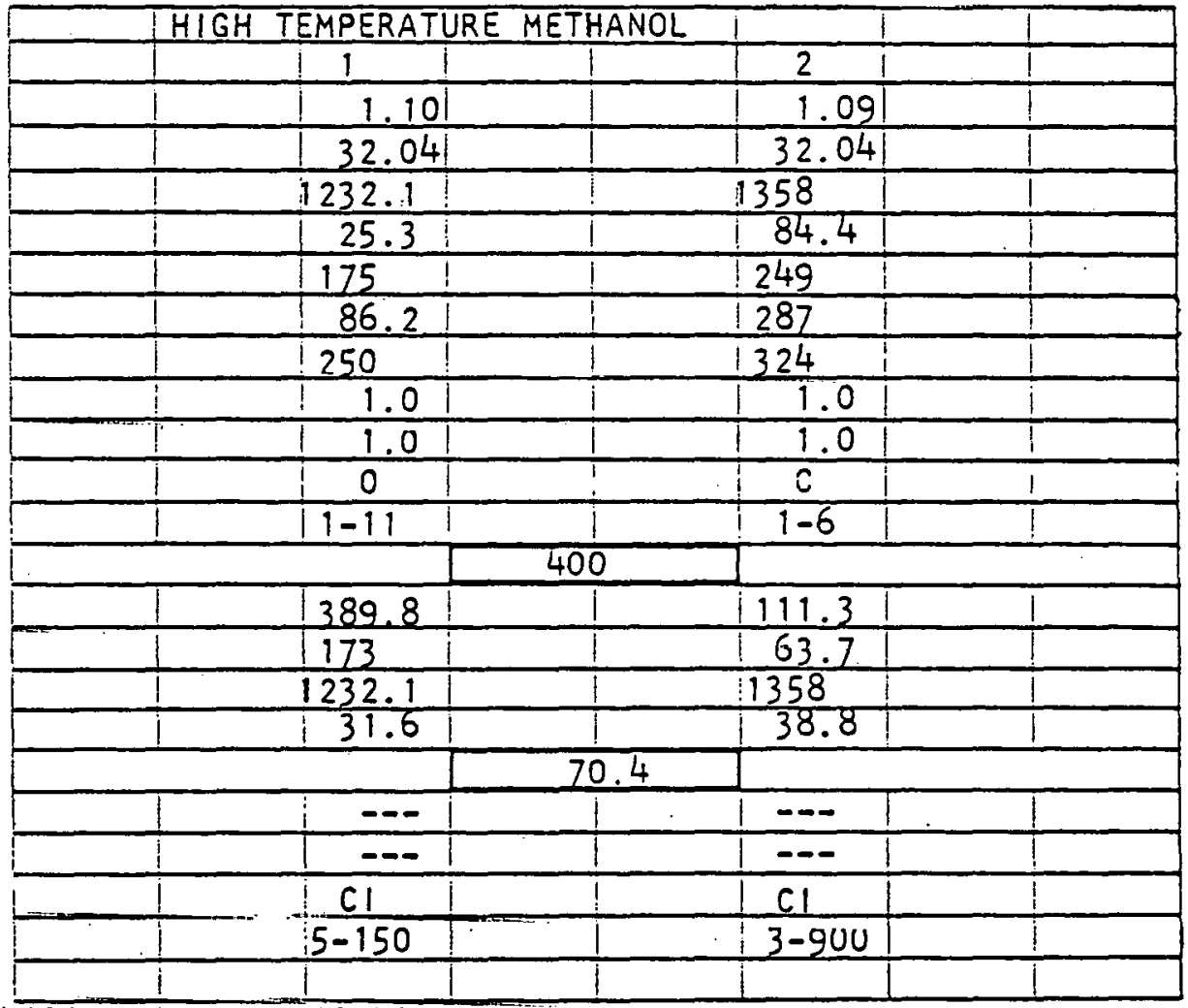

(1) No compressibility data available

Capacify contral - method:

Purchaser's bypass 


\section{Cylinder Data}

Item no./service............. Stage.

No. of cylinders per stage.

Type cylinder.

Single/double acting.

Cylinder liner, yesino.

Cylinder liner, wet/dry..........

O.D. liner, inches .............

Bore, inches..............

Stroke, inches. . . . . . . . . . .

Piston displacement, CFM ........

Clearance, \%.

Volumetric effieiency, $\%, \ldots \ldots \ldots \ldots$

Valve gas speed, FPM

Total no. inlet and discharge volves

Size and type of valves

Piston speed, FPM

Rod diameter, inches.

Piston load at rated conditions, lbs.

Max. allow. piston load, lbs........

Max. allow. eyl press., PSIG.

Recommended relief valve, PSIG...

Hydrostatic test, PSIG:

Inlet size, inches

Inlet ratings, Ibs. (ASA)

Inlet rotings, Ibs. (ASA)..........

Discharge size, inches...........

Discharge rating, Ibs. (ASA).

Cylinders

Cylinder liners.

Pistons.

Pistoon rlngs.

Piston rods.

Valve seats.

Vaive stops

Valve channels or plates..........

Valve springs.............

Compressor Pocking

X Full-floating metallic packing with stainlesssteel springs

X: Force-feed-lubricated

Non-lubricated $[$ TFE $\square$ Carbon

Cooling

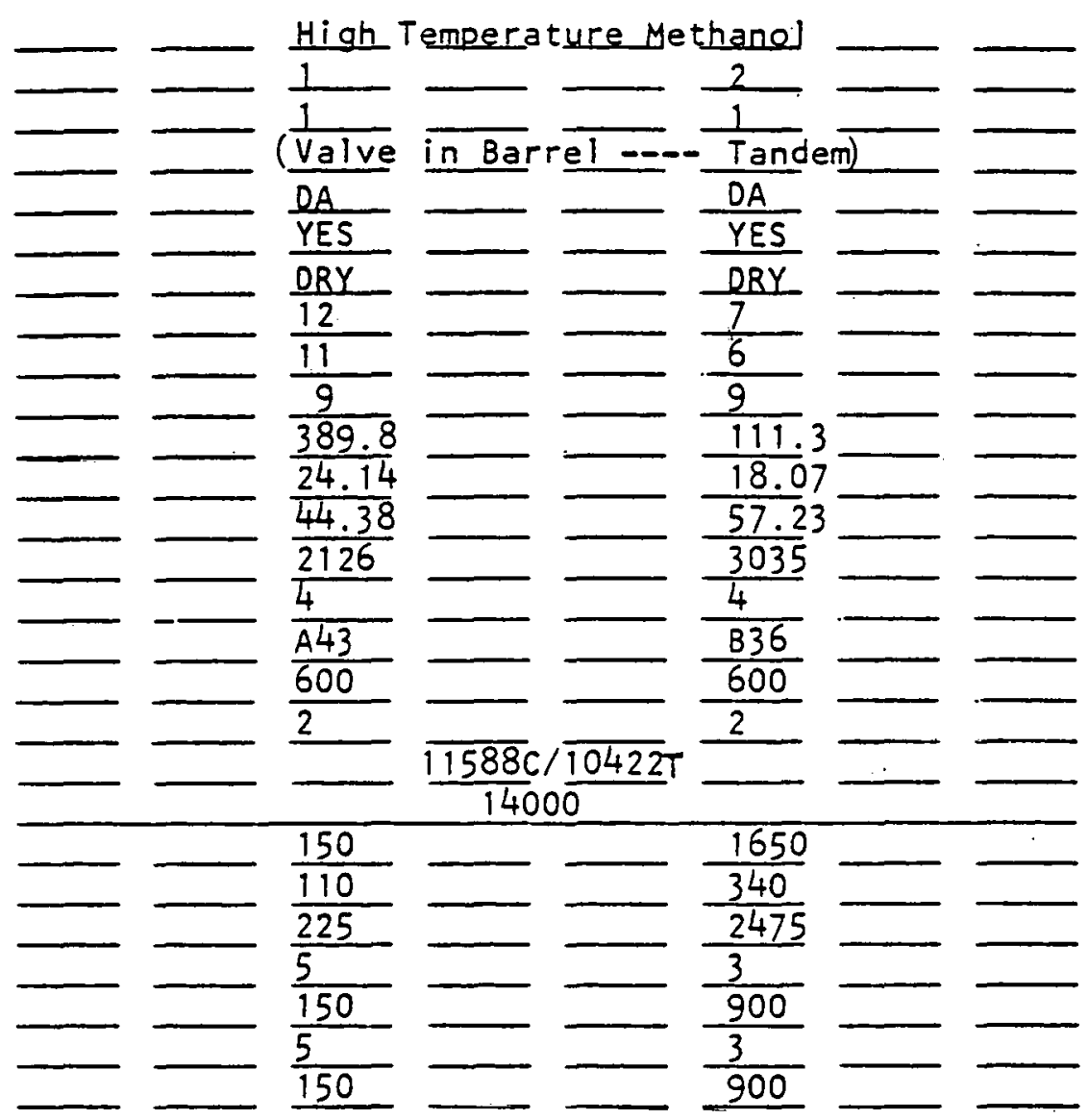

Compressor Materials

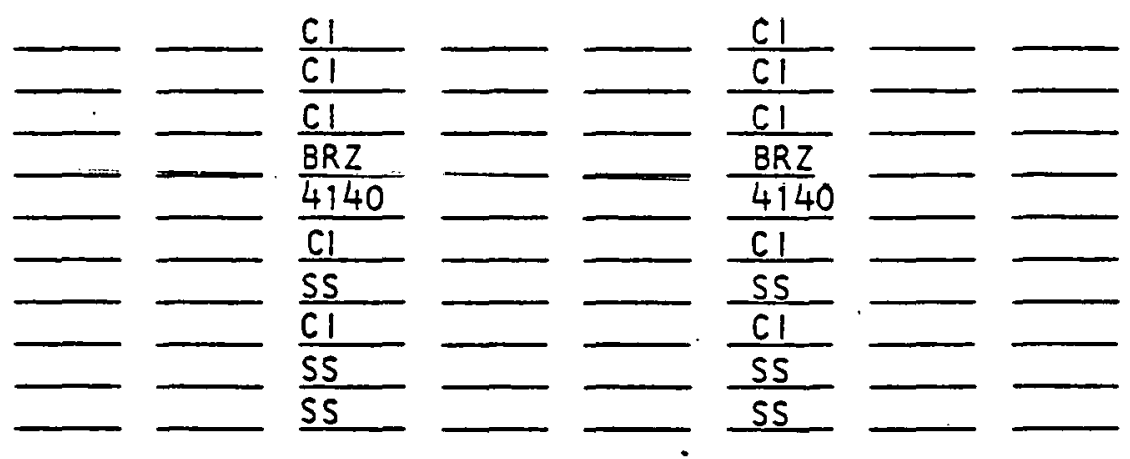

Distance Piece

X Standard length single compartment (Type 1)

$\square$ Extra long single compartment (Type 2)

Two comportment (Type _)

$x$ Solid cover $\square$ Open $\square$ Screened

Cylinder sizes, unless speciffed, are subject to change after final engineering. Other data not shown can be furnished of that time. 


\section{ESH COMPRESSORS}

\section{STANDARDS}

\section{Frame \& Gear}

a. Weathertight - all openings have gasketed covers.

b. No oil piping - all internally drilled thru crankshaft, conn rod, etc.

c. No internal bypass in oil filter.

d. Low oil pressure switches mounted \& piped on unit with SP.DT switch.

e. Built in relief valve and frame breather.

f. Cast iron scrappers.

g. Non-adjustable crosshead, $5^{\prime \prime}$ and 7"

h. Full floating bearing.

i. All bearings are aluminum except crosshead pin is bronze.

II. Lubricator

a. Direct connected to crankshaft.

b. Carbon steel tubing.

c. Check valve at each lube point.

d. Cylinders 15" diameter and larger and all vertical cylinders have two lube feeds. Horizontal cylinder lube in top \& bottom. Vertical cylinders one each side.

e. Lube feed to packing.

III. Packing

a. Lubricated \& vented.

b. Vent piped with earhnn steel tubing from packing to distance piece $1 / 4$ " connection.

c. Steel flange.

d. Held by studs.

(1) e. Lubricated unit has glass filled TFE pressure packing ring except FN-13324A pattern has bronze rings.

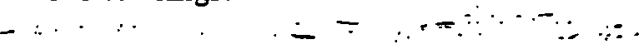

$\because \quad \because=$

g. Packings above 175 psig have cast iron tinnized backup ring except lubricated packing of FN-13324A.

i. Iron asbestos gasket.

IV. Frame Heads

a. Have combination frame head and distance piece on all. 5 \& 7 stroke stock sizes, except FN.13324A, also on the 9 \& 11 stroke cylinder pattems F26174, F26175, F25900.

b. Water jacketed around packing with separate connection from cylinder.

c. Held to cylinder and frame distance piece with studs.

d. Packing box to guide the packing.

(1) Estimate basis metallic packing and piston rings.
V. Cylinders

a. Valve in barrel.

b. Flanged connections.

c. Water jacketed with connection separated from heads, no internal connections of water to heads.

d. Cylinders requiring support have cold support.

e. Head gaskets are asbestos fibre except cylinders rated above 500 psig use flexitallic type.

f. Cylinders without liners have enough metal in bore to remachine $1 / 8^{\prime \prime}$ oversize.

g. Some cylinders have liners as standard - see lineup.

h. Has passages only in cylinder thus no gasketed point to seal inlet from discharge except pattern FN-13324A.

i. Bores have counterbore so piston rings will not wear ridge in cylinder and have trouble removing.

j. Top inlet is standard.

VI. Outer Head

a. Water jacketed (except for pattem 73487) with separate water connection.

b. Held to cylinder with studs.

VII. Vaives

a. Standard vaiving is "A." channel except for pattern FN-13324A and 72839 which uses HS channel.

b. Valve gaskets are lead coated, solid iron gaskets.

VIII. Piston

a. Held to rod by shoulder and nut.

b. Standard pistons up to and including 12" diameter are solid, above $12^{\text {n diameter they }}$ are cored.

c. Cored pistons are supplied with relief plugs.

(2) d. Standard pistons up to and including 7.1/2" diameter are cast iron, above 7-1/2" diameter they are aluminum.

(1) e. Standard piston ring material is glass filled TFE for lubricated except pattern FN-13324A is Micarta and carbon filled TFE with wear band for non-lubricated.

(1) f. Standard TFE-piston rings are one piece expander less angle cut.

(2) g. Aluminum pistons have minovar collars in them to keep expansion of piston and rod the same.

(2) Both pistons to be cast iron. 
IX. Piston Rod

a. Standard rod is AISI 4140 induction hardened to $50 \mathrm{RC}$.

b. Standard finish, in packing travel, is 8 RMS for non-lube and 16 RMS for lubricated.

c. Piston rod is $1-1 / 2$ " diameter for 5 \& 7 stroke

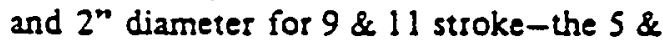
9 stroke rod is necked down at crosshead to $1.1 / 8$ and $1.3 / 4$ respectively, except pattem FN.13324A has $1.1 / 8^{\prime \prime}$ and $1.5 / 8^{n}$ rod on 5 \& 7 respectively.

$\mathrm{X}$ * Water Piping

a. Water is manifolded to one common iniet \& outlet connection for the heads and cylinder.

b. Water piping is copper tubing with sweat fittings supplied with a globe valve on the inlet. c. Water cooled packing has à separate connection on the distance piece and supplied with an injet strainer.

\section{Shipment}

a. ESH units are assembled and given mechanical run test before shipment.

b. Compressor is shipped assembled except for flywheel or belt wheel.

c. Lubricated cylinders are preserved for ship. ment with TECTYL 502-C (purchased from Valvoline Co.) which is equivalent to MIL C.16173C.

d. NL cylinders use VPI crystais or bags in the passage or bore. The rod is wrapped with VPI paper.

e. Domestic units are put on skid for shipment.

* No water piping included. Cylinder water jackets to be filled with insulating material by purchaser and cylinder insulated by purchaser to avoid condensation within cylinder. 


\section{SHOP TEST PROCEDURE FOR ESH, HSE}

1. Level the compressor, install flywheel, sheave and vee belts.

2. Connect flushing pump to compressor.

3. Clean compressor crankcase with clean, lint-free rags.

4. Fill crankease and lubricator with Mobil 524 oil.

5. Loosen lubricator lines at cylinders and pump the lubricator pumps until all lines are full. Tighten lubricator line fittings and pump 20 more times to make sure oil is in cylinders and packings. Connect water line.

6. Cover all frame openings and start the flushing pump.

7. Flush piping for $1 / 2$ hour.

8. Disconnect flushing pump and connect piping for running.

9. Rotate machine at least 3 complete revolutions by hand to make sure there are no interferances in the running gear.

10. Start driving motor and make sure oil comes up to pressure. If oil pressure does not rise to 30 psi in 15 seconds, shut down and find the source of trouble. Check for hot bearings.

11. If compressor runs satisfactorily, run for 5 minutes at full speed. Shut down the compressor and check bearings and running gear to see there is no overheating. Check packing and piston rod for adequate lubrication.

12. If everything is O.K., start up and run for 20 minutes at operating speed, making sure oil pressure is at least 30 psi. Shut down and check for possible overheating as in Step 11.

13. Start up and run for 2 hours.

14. Shut down aild check cylioder bores, piston rods, cranksnait. Feei beariugs to see if any are overheating.

15. Drain crankcase and wipe clean with lint-free rags. Look for metal chips or anything else abnormal. Drain water.

16. Prepare the unit for shipment following instructions covering that operation.

17. Cylinders and pistons should be left on frame for shipment unless order calls for their removal. 
The inlet and discharge vessels or gas piping attached to a compressor cyllnder flange should not introduce excessive forces or moments on the cylinder flange when assembled or in operation. Excessive forces may cause cylinder misalfgnment.

The recommended maximum allowable forces and moments at the cyllnder flange are given below for ESB/HSE units. (The dead weight of the attached vessel can be added to these values). Gas.piping to cylinders or vessels should have supports for its weight.

\section{AIIOWABLE FORCE-MAXIMUM}

Horizontal, $\mathrm{XX}$ axis, parallel to cylinder Vertical, YY axis, vertical to cylinder Horizontal, $2 Z$ axis, perpendicular to cyl.

\section{ALLOWABLE MOMENT-MAXIMUM}

Around $X X$ axis, $Y Z$ plane Around $Y Y$ axis, $X Z$ plane Around $Z Z$ axis, $X I$ plane
FORCE, LBS. $(\mathrm{Kg})$

\begin{tabular}{c|c|c}
\hline 5" or 7" ESH. & $9^{\prime \prime}$ or II" ESH or 9" HSE \\
\hline $\pm 540(245)$ & $\pm 750(341)$ \\
$\pm 430(195)$ & $\pm 600(273)$ \\
$\pm 220(100)$ & $\pm 300(136)$
\end{tabular}

$$
\begin{array}{rlr} 
& \text { MOMENT, LB. FT. }(\mathrm{Kg} . \mathrm{M}) \\
\pm 600 & (83.3) & \pm 900(125) \\
\pm 300 & (41.7) & \pm 450(62.5) \\
\pm 450 & (62.5) & \pm 700(97.2)
\end{array}
$$

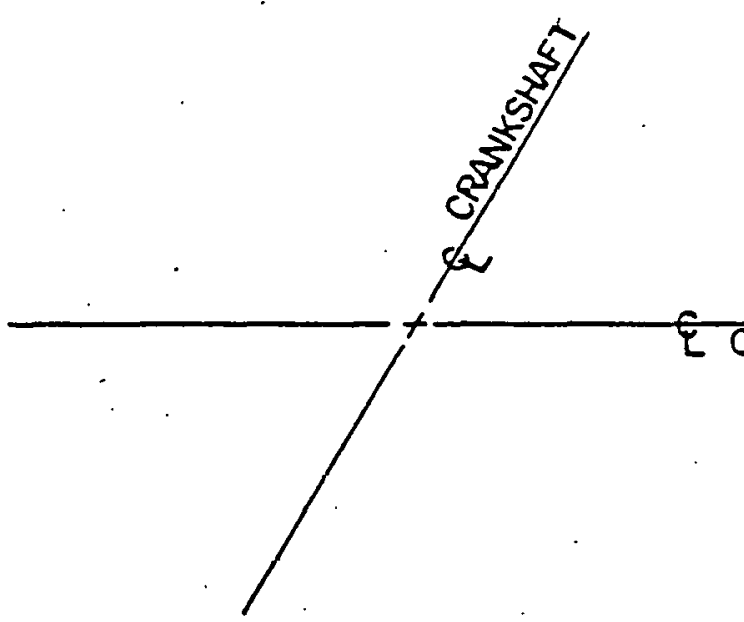

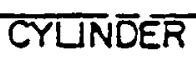

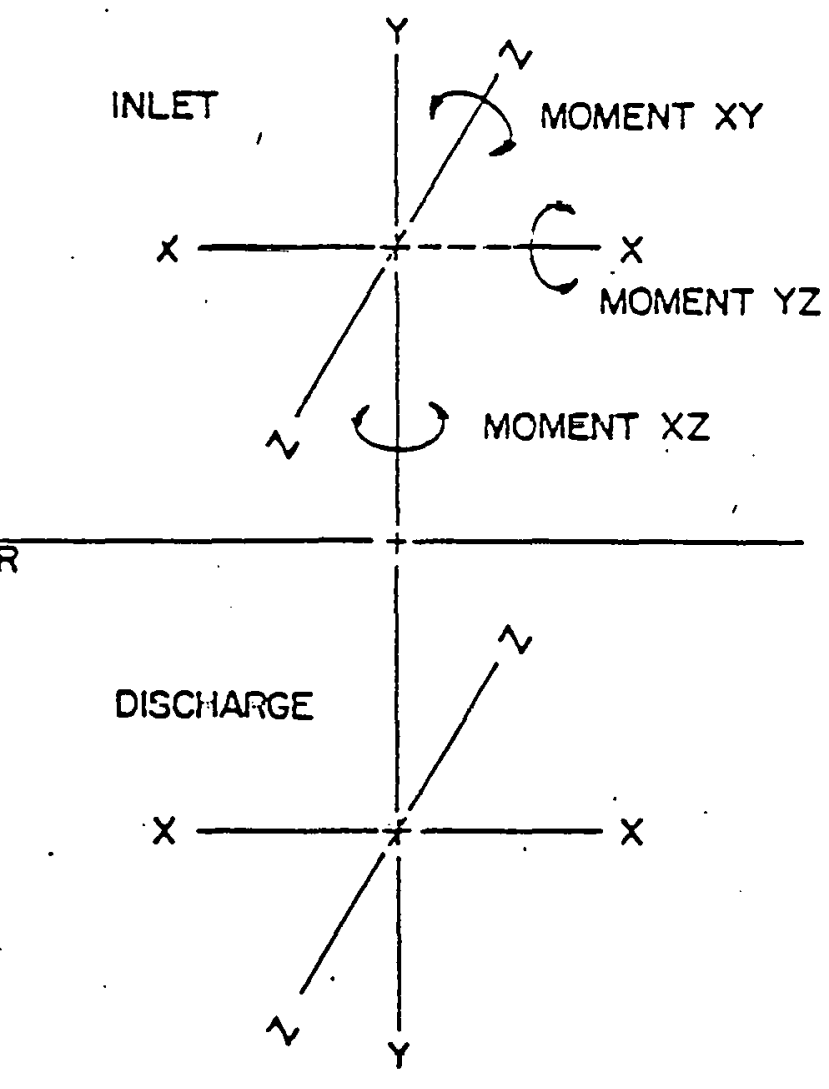

REVISED $1 / 26 / 78$ 


\section{Prices, Terms and Conditions}

Compressor lou ditur.

$\$ 32,588.00$ each

\section{Extra Equipment:}

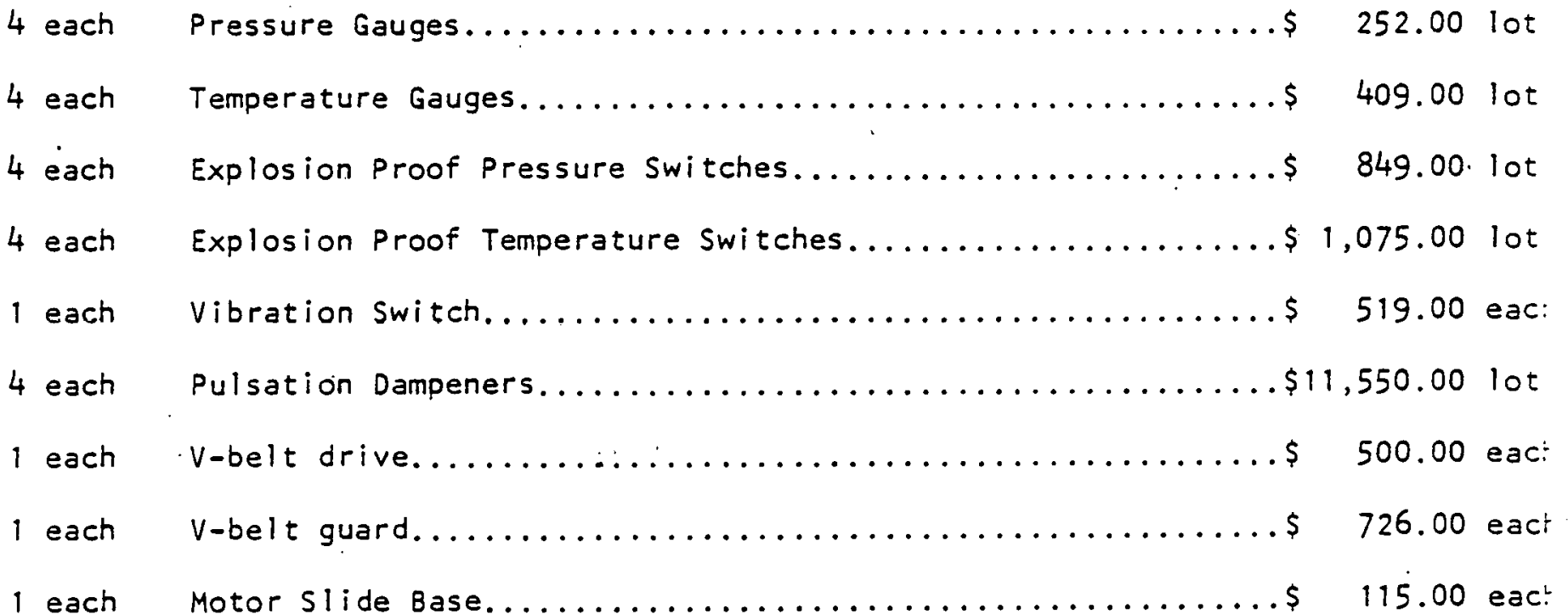

Metallic Packing and $R$ ings....................\$ 1,128.00 lot

\section{Driver}

MOTOR: (Typel Induction
480/3/60 $; \frac{100}{1.0}$ HP; $\frac{1800}{\text { RPM }}$ Rolts/phase/cycles $\frac{1.0}{B}$ P.F.;

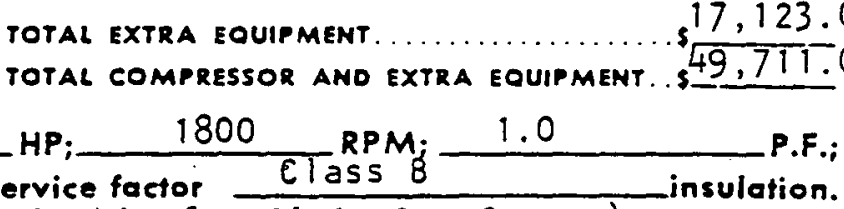

Special features: Explosion Proof Enclosure (Suitable for Cl I, Gr. C area)

TURBINE: (Soleplate)

Price.

GEAR AND HIGH SPEED COUPLING: (soleplate) (common bedplate)

Price.......\$

Price.......\$

Total Price (including extre equipment and driver) $\$ 55,282.00$ each.

F.O.B. Factory Painted Post, New York, and motor vendor's plant.

Erecting Engineer: Sorvices of a Supervisor of Erection are included for zero (0) doys pee compressor, Purchaser to pay man's traveling and living expenses from time he leaves the IngersollRand factory until he returns, and to provide necessary labor and rigging. Purchaser shall pay for services required beyond the days included above.

Terms of Payment: Net Cash 30 days from date of shipment

All payments to be made in U.S. Dollars in New York. Pro rata payments to apply as shipments are made.

Shipment will be made. 24-26 weeks after acceptance of order by an Executive Officer of Ingersoll-Rand Company, Woodeliff Lake, NJ and receipt of complete information necessary for manufacturing.

Confract Provisions: Form LD 101 (attached) is a part of this proposal. 
The Evaporator and Air Heater

The evaporator and air heater are both heat exchangers used to transfer heat between the refrigerant and air streams. These units are of $u$-tube design to reduce the space required at installation and provide the heat transfer characteristics desired. The following pages contain the design information provided and the quotations received from American Standard. Due to their complex nature these units must be constructed by experienced manufacturers. 


\subsection{6}

Heat Exchanger $\# 3$ is a Cross Flow Finned Tube Heat Exchanger with evaporating methianol on the tube side and air-water vapor mixture on the shell side. This Heat Exchanger condenses part of the water vapor out of the air stream which must be removed as condensate.

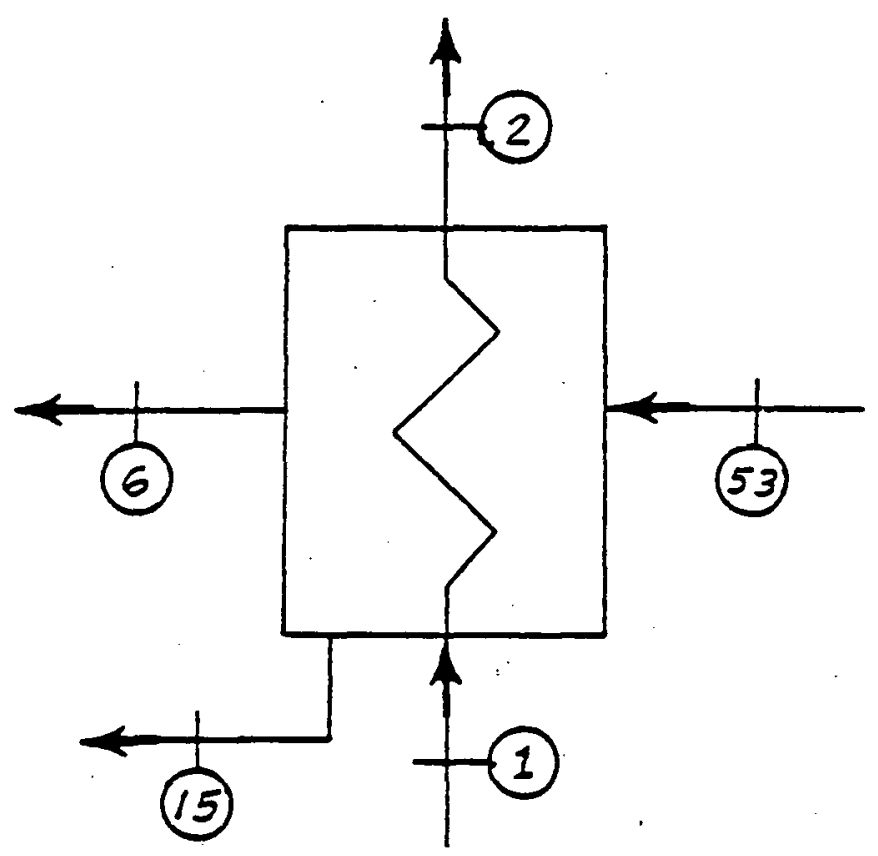

\section{STATE 1}

$T$ T $=175^{\circ} \mathrm{F}$

$P T=25.6$ PSIA

$\mathrm{hl}=146 \mathrm{BTU} / \mathrm{Tbm}$

$\dot{M} 1=1325 \mathrm{lbm} / \mathrm{Hr}$

$\dot{v} 1=1205 \mathrm{Ft}^{3} / \mathrm{Hr}$

SATURATED MIXTURE OF LIQUID AND VAPOR

METHANOL
STATE 2

$\mathrm{T} 2=175^{\circ} \mathrm{F}$

$P 2=25.6$ PSIA

$\mathrm{h} 2=549 \mathrm{BTU} / \mathrm{ibm}$

$\dot{M} 2=1235 \mathrm{Ibm} / \mathrm{Hr}$

$\dot{v} 2=9635 \mathrm{Ft}^{3} / \mathrm{Hr}$

SATURATED VAPOR

METHANOL 


\section{STATE 6}

$T 6=195^{\circ} \mathrm{F}$

$P 6=14.7-$ PSIA

$\mathrm{RH} 6=100 \%$

$h W 6=1144 \mathrm{BTU} / 1 \mathrm{bm}$

$h A 6=39.1 \mathrm{BTU} / 1 \mathrm{bm}$

M $W 6=2900 \mathrm{ibm} / \mathrm{Hr}$

$\dot{M A G}=1900 \mathrm{ibm} / \mathrm{Hr}$

$\dot{v}_{\sigma}=108,410 \mathrm{Ft}^{3} / \mathrm{Hr}$

AIR-WATER VAPOR MIXTURE

\section{STATE 53}

$T 53=205^{\circ} \mathrm{F}$

$P 53=14.7+P S I A$

$\mathrm{RH} 53=85 \%$

$h W 53=11498 \mathrm{TUU} / 1 \mathrm{bm}$

$h A 53=41.52$ BTU $/ 1 \mathrm{bm}$

$\dot{M} W 53=3400 \mathrm{lbm} / \mathrm{Hr}$

$\dot{M}$ A53 $=19001 \mathrm{bm} / \mathrm{Hr}$

$\dot{v} 53=123,493 \mathrm{Ft}^{3} / \mathrm{Hr}$

AIR-WATER VAPOR MIXTURE

STATE 15

$T 75=195^{\circ} \mathrm{F}$

P15 $=14.7$ PSIA

$\mathrm{h} 15=163 \mathrm{BTU} / \mathrm{Tbm}$

$\mathrm{h} / 5=500 \mathrm{1bm} / \mathrm{Hr}$

Vi15 $=8.3 \mathrm{Ft}^{3} / \mathrm{Hr}$

LIQUID WATER

HEAT RELEASE RATE

$\dot{Q}=497,700 \mathrm{BTU} / \mathrm{Hr}$ 
Heat Exchanger $\# 2$ is a Counter Flow Finned Tube Heat Exchanger with liquid methanol on the tube side and air-water vapor mixture on the shell side. This Heat Exchanger is to be used as an Air Heater.

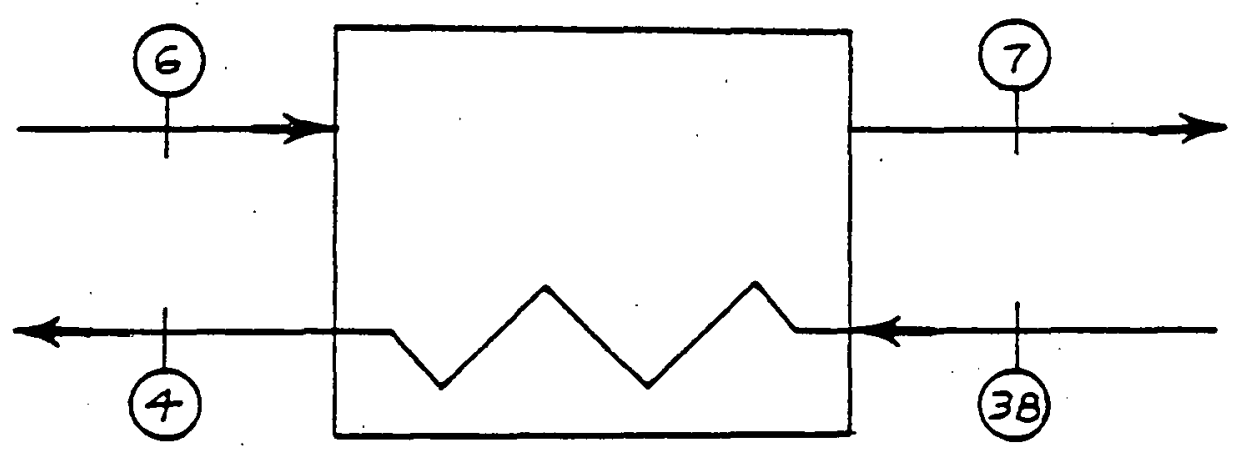

\section{STATE 6}

$T 6=195^{\circ} \mathrm{F}$

P6 $=14.7^{+}$PSIA

RH6 $=100 \%$

$h W 6=11448 T \mathrm{TU} / 1 \mathrm{bm}$

hAG $=39.1 \mathrm{BTU} / 1 \mathrm{bm}$

$\dot{M} W 6=2900 \mathrm{lbm} / \mathrm{Hr}$

MAE $=19001 \mathrm{bm} / \mathrm{Hr}$

$\dot{v} \sigma=108,410 \mathrm{Ft}^{3} / \mathrm{Hr}$

AIR-WATER VAFOR MIXTURE
STATE 7

$T 7=248^{\circ} \mathrm{F}$

$P 7=14.7^{-}$PSIA

$\mathrm{RH} 7=36 \%$

$h W 7=1169 \mathrm{BTU} / \mathrm{Tbm}$

$h A 7=51.84 \mathrm{BTU} / 1 \mathrm{bm}$

$\dot{M} W 7=2900 \mathrm{ibm} / \mathrm{Hr}$

MA7 $=19001 \mathrm{bm} / \mathrm{Hr}$

$\dot{v}_{i}=117,125 \mathrm{Ft}^{3} / \mathrm{Hr}$

AIR-WATER VAPOR MIXTURE 


\section{STATE 33}

$T 3 B=330^{\circ} \mathrm{F}$,

$P 3 B=284 P S I A$

$h 3 B=217.2 B T U / 1 b m$

$\dot{M} 3 B=13601 \mathrm{bm} / \mathrm{Hr}$

$\dot{V} 3 B=35 \mathrm{Ft}^{3} / \mathrm{Hr}$

SATURATED LIQUID

METHANOL

HEAT RELEASE RATE $\dot{\mathrm{Q}}=96,560 \mathrm{BTU} / \mathrm{Hr}$

\section{STATE 4}

$T 4=248.9^{\circ} \mathrm{F}$

$P 4=284$ PSIA

$\mathrm{h} 4=146 \mathrm{BTU} / 1 \mathrm{bm}$

$\dot{M} 4=13601 \mathrm{bm} / \mathrm{Hr}$

$\dot{\mathrm{V}}_{4}=32 \mathrm{Ft}^{3} / \mathrm{Hr}$

LIOUID METHANOL 
HEAT TRANSFER DIVISION

AMERICAN-STANDARD

BUFFALO. N.Y. 14240

\section{HEAT EXCHANGER SPECIFICATION SHEET}

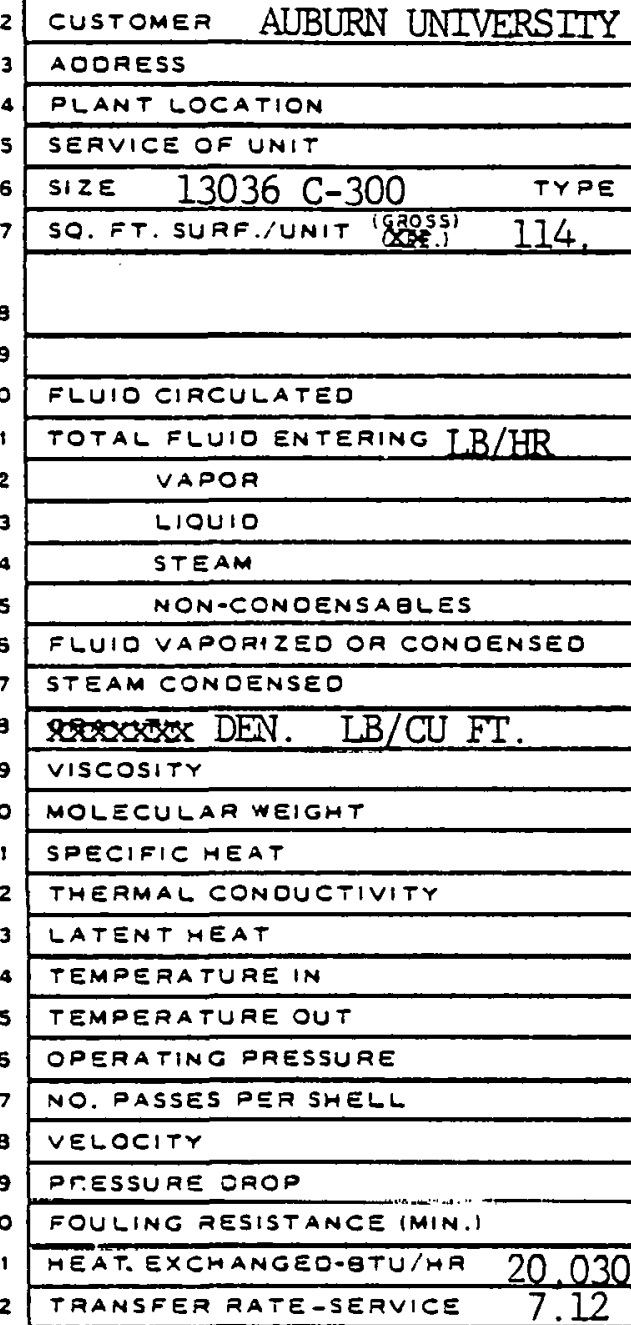

\section{PERFORMANCE OF ONE UNIT}

\begin{tabular}{c|c} 
SHELL SIDE & TUBE SIDE \\
\hline ATR/WATER & METHNOL, VAPOR \\
\hline 5300 & 1235 \\
\hline
\end{tabular}

JOB NO

REFERENCENO.

INOUIRY NO. $1279 A 095$

OATE I/17/80

ITEM NC. 0202

(HORI2.) CONNECTEO IN

ONE SO. FT. SURF.'SHELL lGROSSI 114
1235

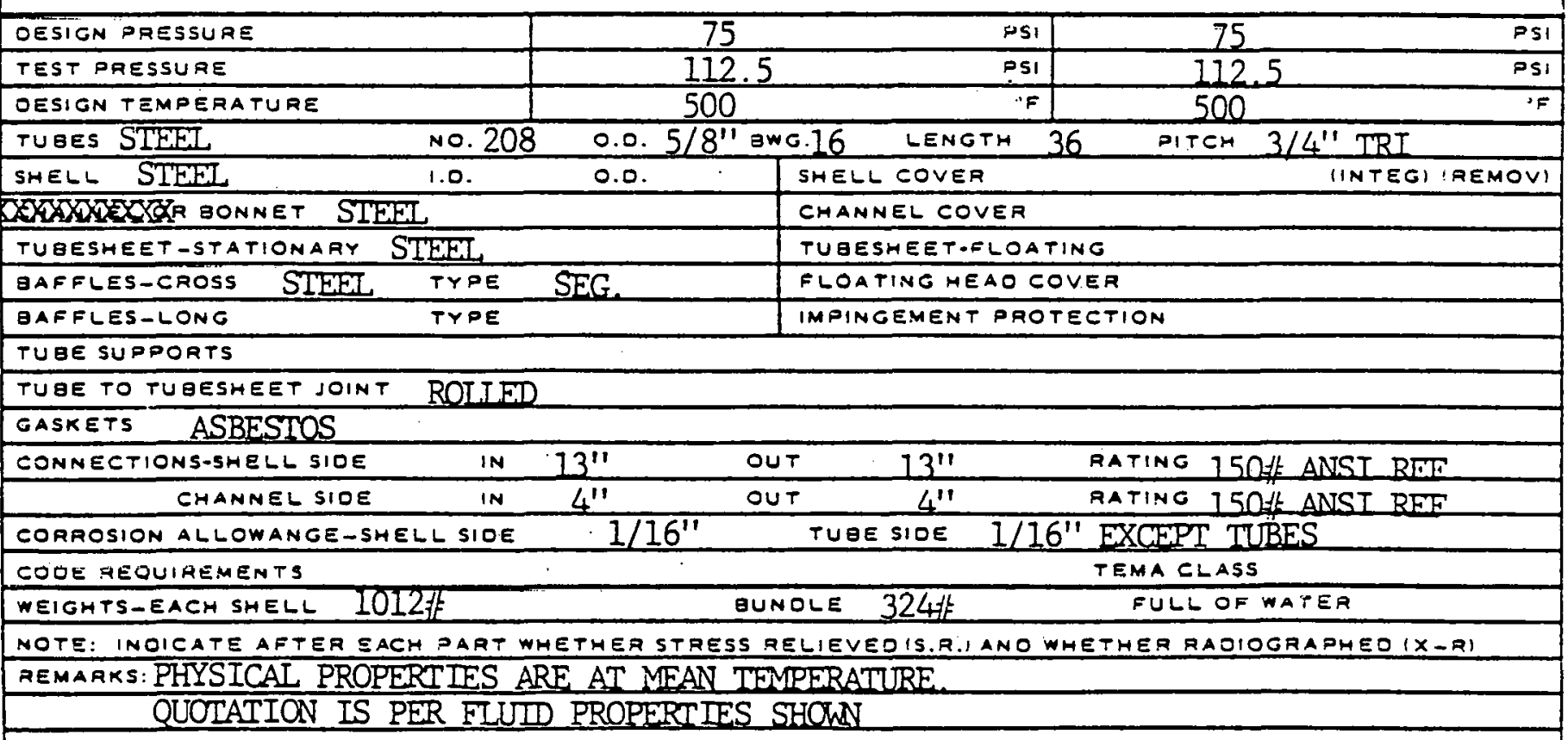

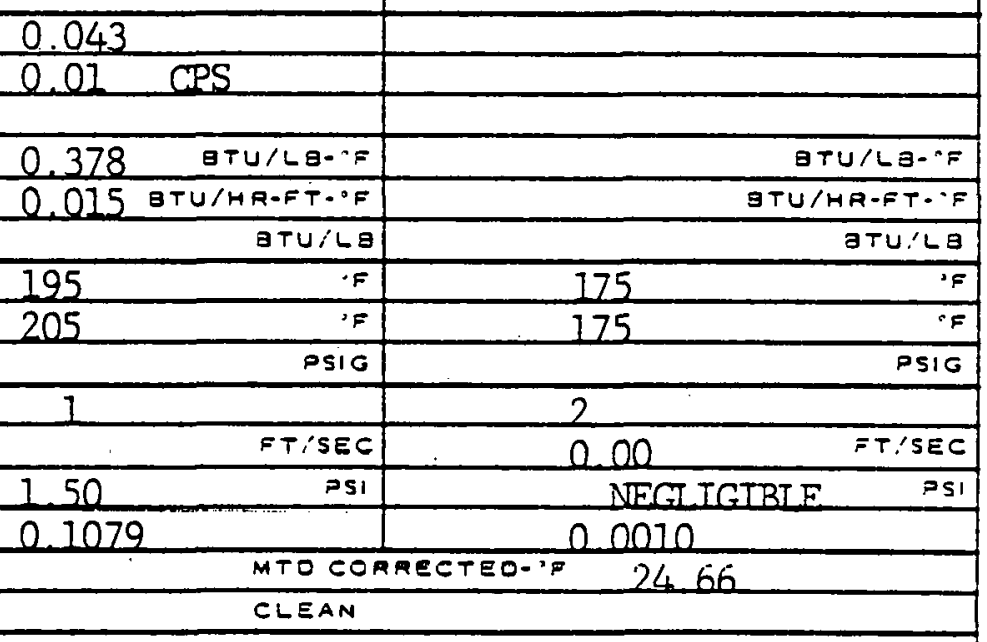

CONSTRUCTION OF ONE SHELL 


\section{HEAT EXCHANGER SPECIFICATION SHEET}

\begin{tabular}{|c|c|c|c|}
\hline 1 & \multirow{2}{*}{\multicolumn{2}{|c|}{ CUSTOMER AUUBRN UNIVERSITY }} & JOBNO \\
\hline 2 & & & REFERENCENO. \\
\hline 3 & \multicolumn{2}{|l|}{ AODRESS } & INOUIRY NO. $1279 A 094$ \\
\hline 4 & \multicolumn{2}{|l|}{ PLANT LOCATION } & $1-17-80$ \\
\hline s & \multicolumn{2}{|l|}{ SERVICE OF UNIT } & ITEMNO. \\
\hline 6 & \multicolumn{3}{|c|}{ SIzE $\quad 12042$ C-300 TYPE U-tube } \\
\hline 7 & \multicolumn{3}{|c|}{ SO. FT. SURF.'UNIT IERSTI 108.5 SHELLSIUNIT } \\
\hline a & \multicolumn{3}{|c|}{ PERFORMANCE OF ONE UNIT } \\
\hline 9 & & SHELL SIDE & TUBE SIOE \\
\hline 10 & FLUIO CIRCULATEO & AIR/WATER & METHANOL \\
\hline$\because 1$ & TOTAL FLUID ENTERING IR/HR & 4800 & 1360 \\
\hline 12 & VAPOA & & \\
\hline 13 & L10U10 & & \\
\hline 14 & STEAM & & \\
\hline is & NON-CONOENSABLES & & \\
\hline 16 & FLUIO VAPOAIZEO OA CONDENSED & & \\
\hline 17 & STEAM CONDENSED & & \\
\hline 18 & DEN IB/CU ET. & 0.043 & 48 \\
\hline 19 & VISCOSITY & 0.02 CPS & 0.10 \\
\hline 20 & MOLECULAR WEIGHT & & \\
\hline 21 & SPECIFIC HEAT & BTU/LB:-F & BTU/LB-`F \\
\hline 22 & THERMAL CONOUCTIVITY & 0.015 BTU/HR-FT. ${ }^{\circ} \mathrm{F}$ & O.097 BTU/HR-FT-AF \\
\hline 23 & LATENT HEAT & BTU/LO & BTU.LS \\
\hline 24 & TEMPERATURE IN & 195 & 330 \\
\hline 25 & TEMPERATURE OUT & 248 & 348.90 \\
\hline 26 & OPERATING PRESSURE & PSIG & PSIG \\
\hline 27 & NO. PASSES PER SHELL & 1 & 4 \\
\hline 28 & VELOCITY & FT:SeC & FT!SEC \\
\hline 29 & PTESSURE DROP & PSI & PSI \\
\hline 30 & FOULING RESISTANCE IMIN.) & 0.0022 & 0.0010 \\
\hline 31 & REAT. EXCHANGED-ETU/HR & MTOCOF & ARECTEO-'F \\
\hline 32 & TRANSFER RATE-SERVICE & CLEAN & \\
\hline 33 & & OONSTRUCTION OF ONE SHELL & \\
\hline 34 & DESIGN PRESSUAE & PSi & 300 \\
\hline 35 & TEST PRESSURE & 112.5 & $450^{\circ}$ \\
\hline 36 & OESIGN TEMPEAATURE & 500 & 500 \\
\hline 37 & TUBES WLD S/S & $0.0 .5 / 8$ gwG. 16 & $3 / 4^{\prime \prime}$ TRI \\
\hline 38 & SHELL STEEI, & SHELL COVER & IINTEG) IREMOVI \\
\hline 39 & SWOMOEOXXR OONNET & CHANNEL COVER & \\
\hline so & TUEESHEET-STATIONAAY & TUBESHEET-FLOA & TING \\
\hline 41 & BAFFLES-CROSS STEET, & FLOATINC HEAOC & EOVER \\
\hline 42 & BAFFLES-LONG & IMPINGEMENT PRO & DTECTION \\
\hline 43 & TUAE SUPPOATS & & \\
\hline 44 & TUQE TO TUQESHEET JOINT & & \\
\hline 45 & GASKETS ASBESTOS & & \\
\hline 46 & EONNEETIONS-SHELL SIOE & $12^{\prime \prime}$ & RATINC 150 \\
\hline 47 & CHANNEL SIOE & OUT & RATING 300 AE ANSI REF \\
\hline 48 & CORROSION ALLOWANGE-SHELL SIDE & TUBE SIOE $1 / 16$ & 6" EXCEPT TUBES \\
\hline 49 & COOE AEOUIREMENTS & & TEMACLASS \\
\hline so & WEIGHTS-EACH SHELL & BUNOLE & FULL OF WATEA \\
\hline 51 & NOTE: INOICATE AFTER EACM PART H & VHETHER STRESS RELLIEVEO IS.A.IANO V & WHETHER RAOIOGRAPHEO!X-RI \\
\hline 32 & REMARKS: PHYSICAL PROPERTIE: & S ARE AT MEALI TEMPERATURE & \\
\hline & QUOTATION IS PER EI & LUTD PROPERTIES SHOWN & \\
\hline
\end{tabular}


OAi J: January 17,1980

REFEลENCE: Am. Std. Inq. \#1279 A095

AUBURN UNIVERSITY

Dept. of Mech. Engr.

Aubumn, Alabama 36830

Attention: Mr. Tom Birch

Gentlemen:

We are pleased to offer our quotation on your incuirl as referenced above.

One (I) \#12048 C-300 unit... . \$3,834.00 net each, F.O.B. Buffalo, New York

One (1). 非13036 C-300 unit . . . \$4,264.00 net each, F.O.B. Buffalo, inew York

DESCRIPTION. $\quad$ Specification sheetsattached. Bulletin Attached.

DATA :

When ordering, advise nozzle orientation desired

Drawings for approval can be submitted inithin

days following receipt of order.

to

Norking

Exception and/or clarifications to submitted specifications are shown on the attached sheet

2RICING: $\quad$ XXrices are firm for acceptance within 30 days from the date of quotation and are firm provided shipment is requested or is acceptable within NINE $(9)$ months from the date of quotation. If shipment is requested or ielayed by the purchaser of for any other reason beyond this period, the prices quoted are subject to adjustment to conform to the latest prices in effect at the time of shipmert.

TERMS OF PAYMENT

Q Net 30 days. After 30 days from date of invoice, a Is per month service charge will be added. A 2 s per month service charge will be added to all invoices over 60 days.

Other

DEIIVERY: $\square$ shipment from stock

E $\frac{18}{18}$ to 20 weeks Eollowing receipt of drawirg approval and or release for manufacture

Should you require any additional assistance and for orcer placement in regarc to this quotation, you may contact, Mr. Nick Phillips of our Birmingham Sls. Office(205)663-5086. $\mathrm{JB} / \mathrm{dw}$

cc: N. Phillips-Binmingham

T. O'Neal-Atlanta Reg.

Very truly yours.

HEAT TRANSFER DIYLSION 
The Accumulator Tanks

The accumulator tanks are pressure vessels equipped with spray nozzles that are used to saturate the superheated refrigerant at the compressor exits. These tanks are to be equipped with level controllers provided by the Foxboro Company. They are to be constructed by the Auburn University Machine Shop with purchased materials and parts. The construction details of these tanks are shown in the following pages. 
Accumulator tank $\# 1$ contains saturated liquid methanol at $248.9^{\circ} \mathrm{F}$.

This tank is used to saturate the vapor from the iirst stage of compression in order to reduce the work required for the second stage of compression.

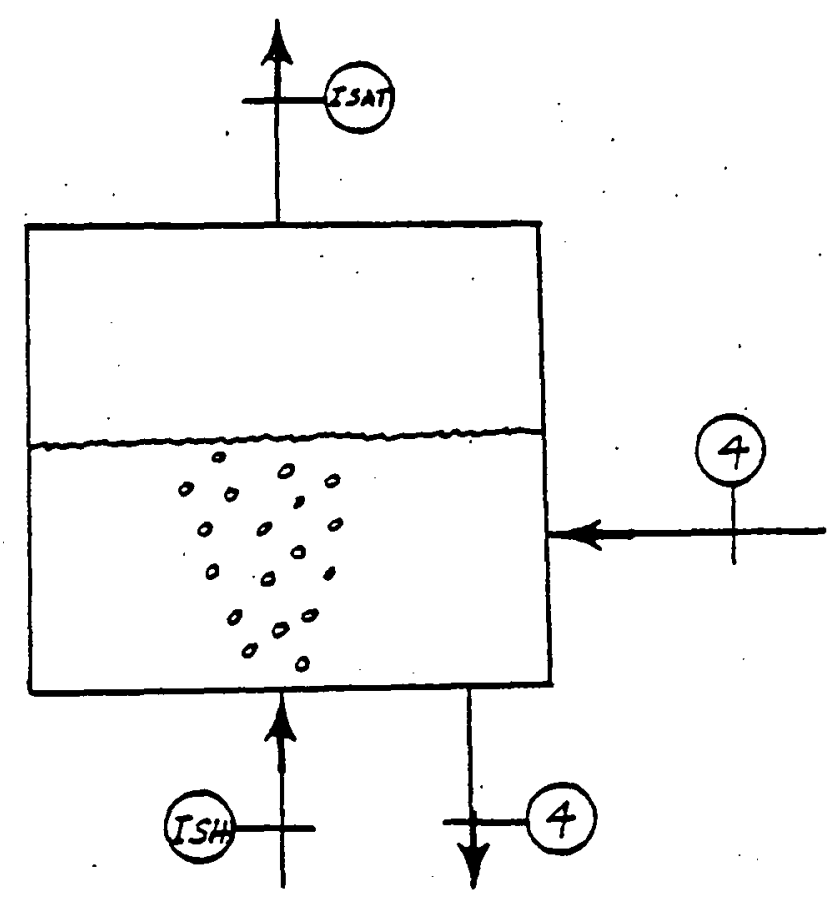

STATE ISH

STATE ISAT

$T I S H=2970 \mathrm{~F}$

TISAT $=248.9^{\circ} \mathrm{F}$

PISH $=85.3$ PSIA

PISAT $=85.3$ PSIA

$\mathrm{hISH}=615 \mathrm{BTU} / 1 \mathrm{bm}$

hISAT $=5608 T \mathrm{~J} / 1 \mathrm{bm}$

$\dot{M} I S H=12351 \mathrm{bm} / \mathrm{Hr}$

MISAT $=1360 \quad 16 \mathrm{~m} / \mathrm{Hr}$

$\dot{V} I S H=3735 \mathrm{Ft}^{3} / \mathrm{Hr}$

VISAT $=2900 \mathrm{Ft}^{3} / \mathrm{Hr}$

SUPERHEATED VAPOR

SATURATED VAPOR

METHANOL

METHANOL 
STATE 4 IN

$T 4=248.9^{\circ} \mathrm{F}$

$P 4=85.3$ PSIA

$h 4=146 \mathrm{BTJ} / 1 \mathrm{bm}$

$\dot{M} 4 \mathrm{IN}=13601 \mathrm{bm} / \mathrm{Hr}$

V $4 \mathrm{IN}=32 \mathrm{Ft}^{3} / \mathrm{Hr}$

SATURATE LIQUID

METHANOL
STATE 4 OUT

$T 4=248.9^{\circ} \mathrm{F}$

$P 4=85.3$ PSIA

$h 4=1468 T U / 16 m$

$\dot{M} 40 U T=13351 \mathrm{bm} / \mathrm{Hr}$

V4OUT $=31.4 \mathrm{Ft}^{3} / \mathrm{Hr}$

SATURATED LIOUID

METHANOL

Accumulator Tank $\frac{\mu}{\pi} 2$ contains saturated liquid methanol at $330^{\circ} \mathrm{F}$. This tank is used to saturate the vapor from the second stage of compression in order to facilitate heat transfer in Heat Exchanger ABOT.

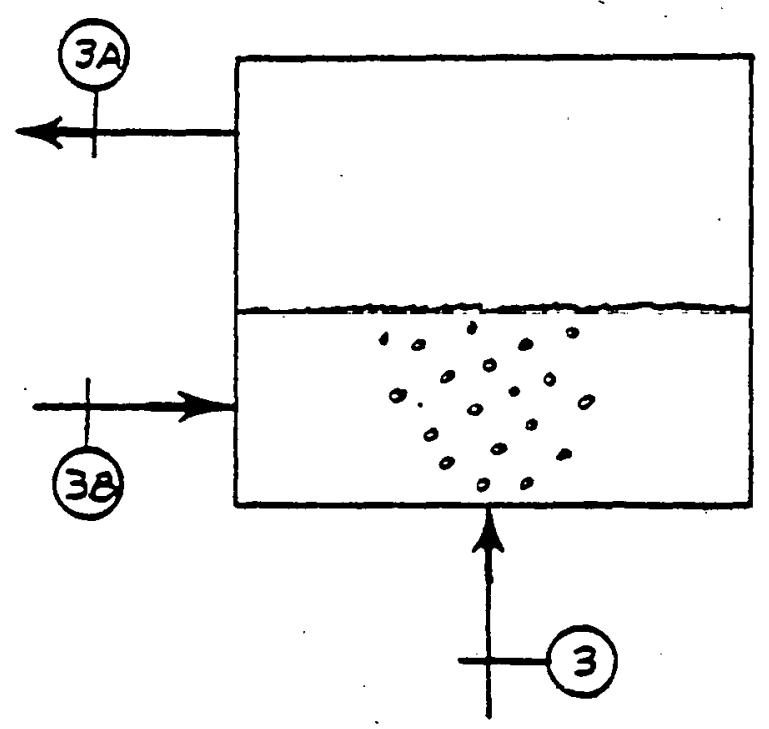




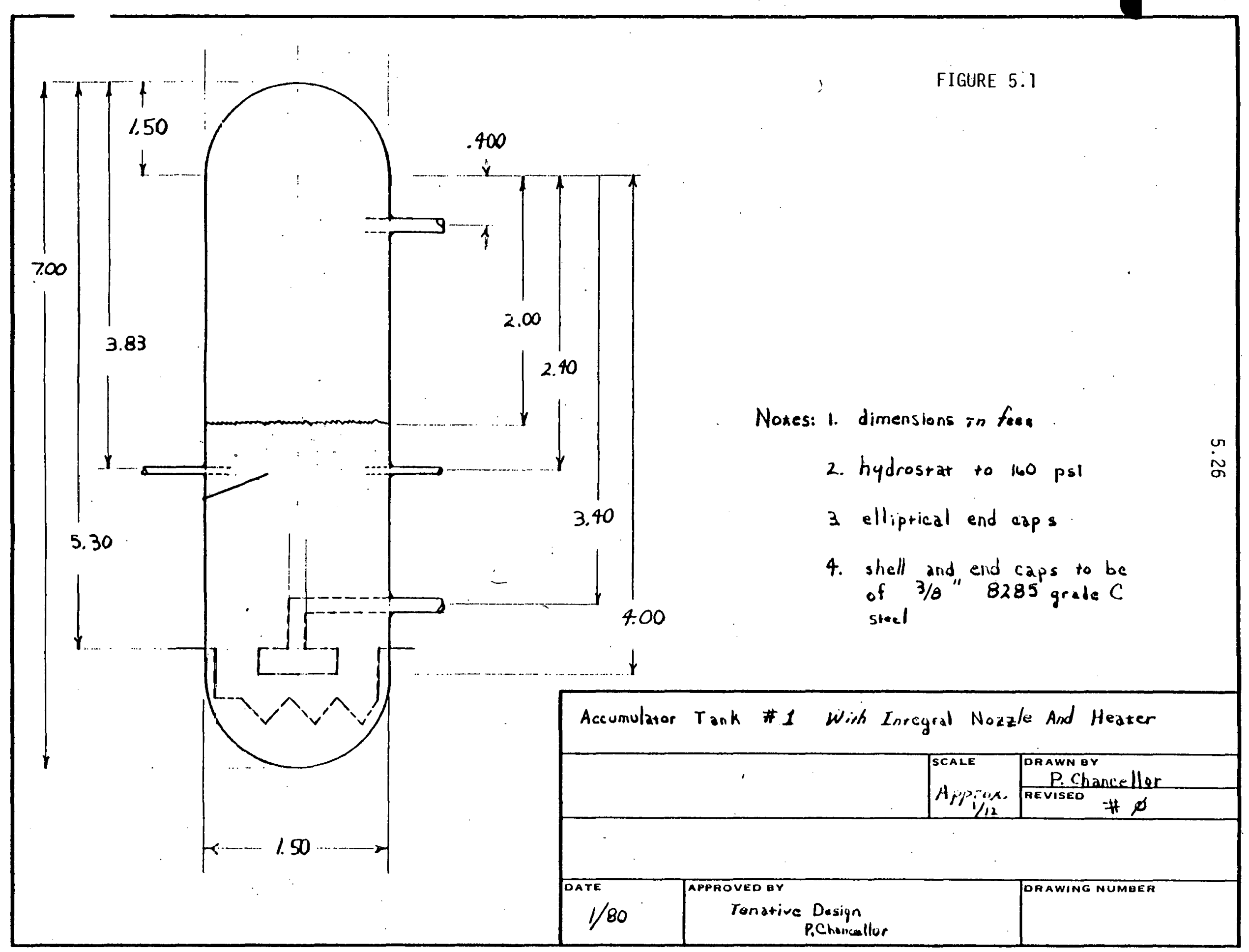




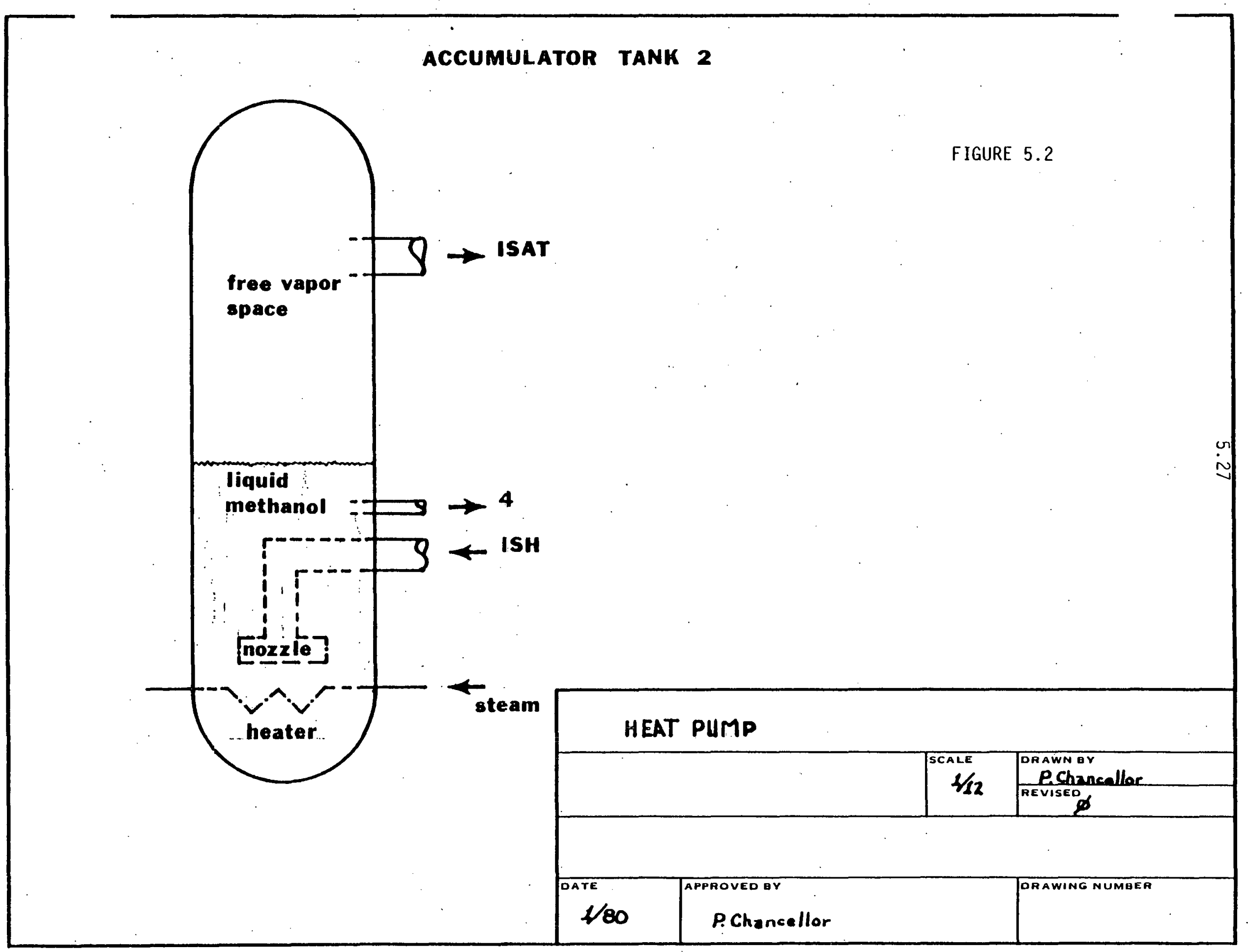




\section{The Oryer}

For the demonstration model a simulated dryer will be used to reduce cost. The requirements of the simulated dryer are: 1) reproduce the flow rate of water admitted to the air stream by an actual dryer, 2) simulate the energy flows present in an actual dryer, and 3) produce dryer exhaust conditions that are typical of industrial dryers. These conditions are met by the simulated dryer design shown in the following pages. The dryer design is fairly simple and can be constructed on site using commercially available materials. 
FIGURE 5.3 .

\section{CUTANAY OF DRYER SIMULATOR AND INTEGRAL BOILER.}

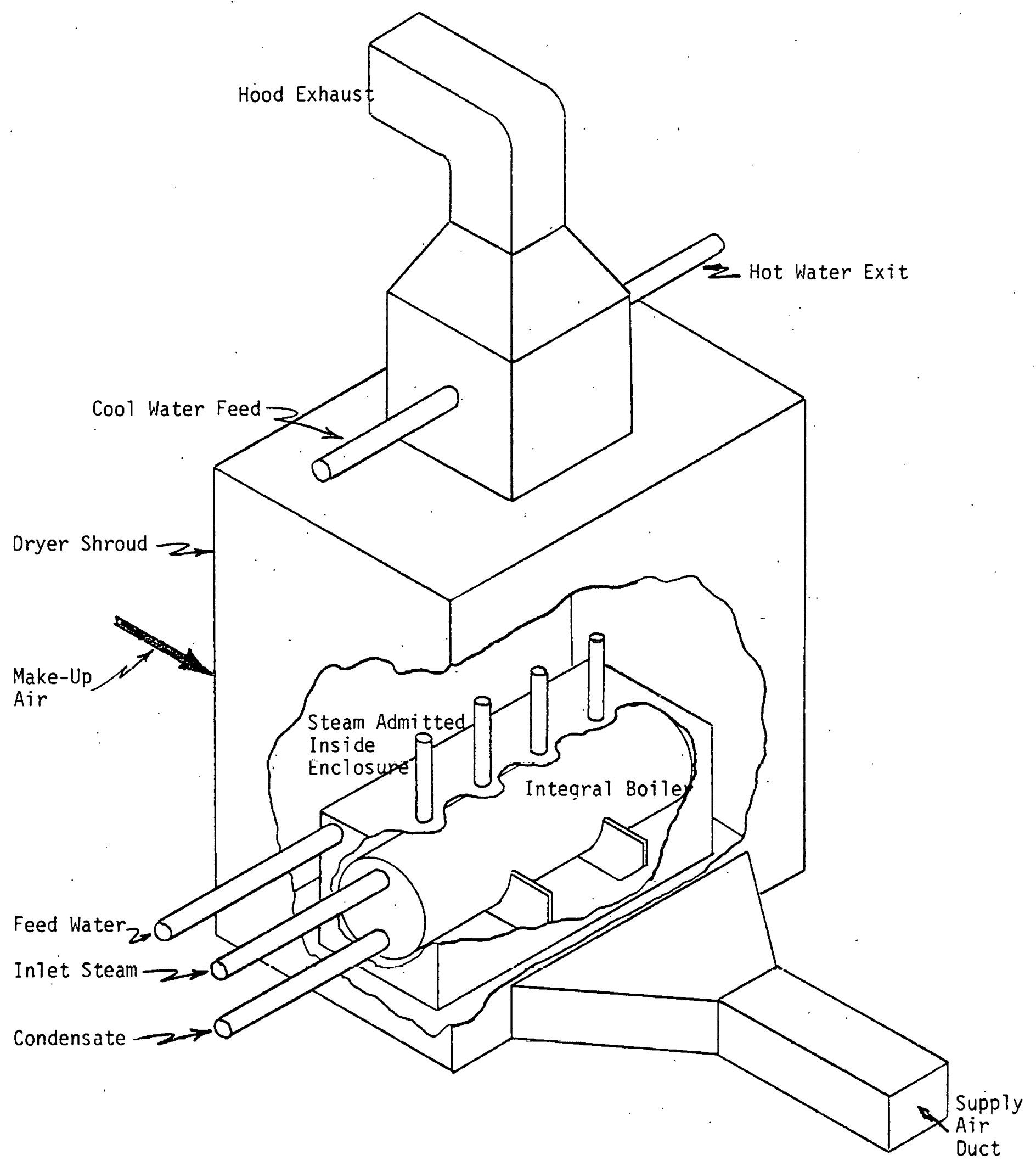


5.30

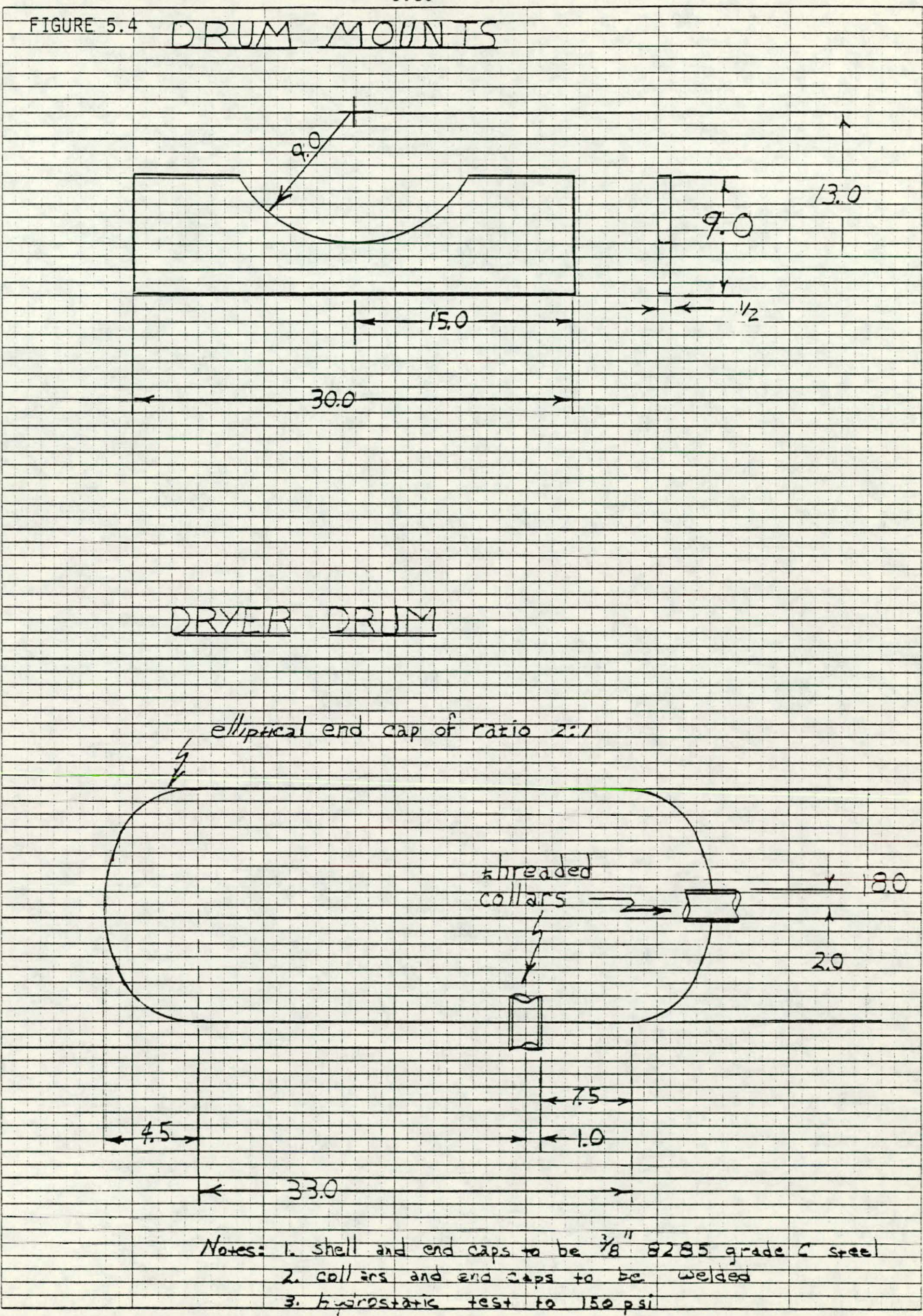


FIGURE 5.5- BOILER WIATER IANK

Notes: 1. $1 / s^{1 n}$ steel plate but welód 2 bottom plate as indichted $\left(30^{\prime \prime} \times 50^{\prime \prime}\right)$ 


\section{The Condenser}

The condenser is a shell and tube heat exchanger used to generate steam for the drying process. It is a one-pass design with the tubes placed in the lower portion of the shell giving $40 \%$ free vapor space on the shell side. The following pages contain information provided for design and the quotation received from Pfaudter Corporation. The construction of this unit is fairly simple except for the expansion joint that must be provided for system start-up and can probably be built in the Auburn University Machine Shop with purchased parts. 
1. Heat Exchanger $\# 1$ is a Shell and Tube type Heat Exchanger with condensing methanol vapor on the tube side and boiling water on the shell side. This heat exchanger is to be used as a steam generator.

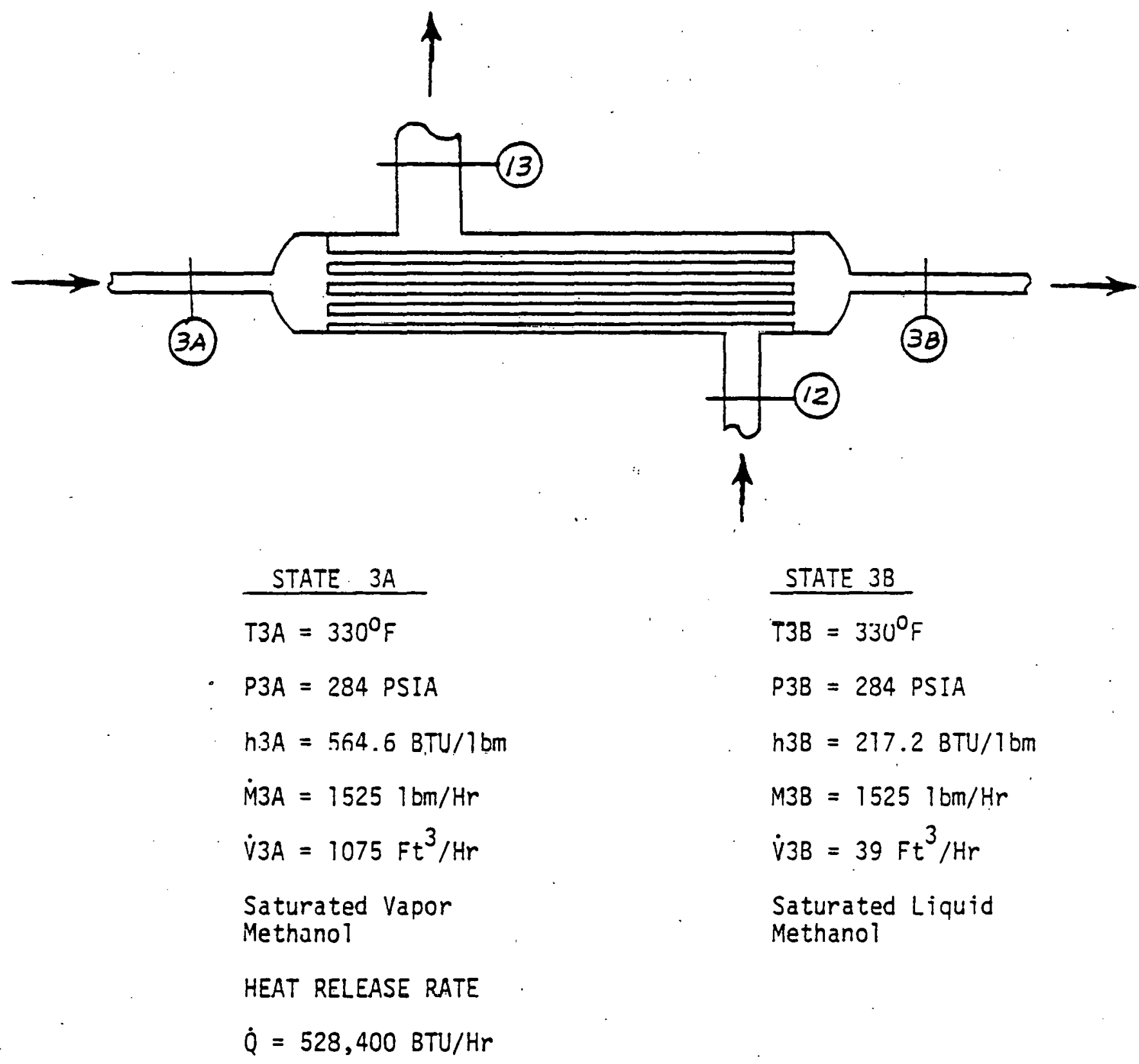


5.34

STATE 12

$\mathrm{T} 12=302^{\circ} \mathrm{F}$

P12 = 75 PSIA

$\mathrm{h} 12=271.75 \mathrm{BTU} / \mathrm{lbm}$

$\dot{M} 12=585 \cdot 1 \mathrm{bm} / \mathrm{Hr}$

$\dot{v}_{12}=11 \mathrm{Ft}^{3} / \mathrm{Hr}$

LIQUID WATER
STATE 13

$T 13=307^{\circ} \mathrm{F}$

P13 = 75 PSIA

$h 13=1182.4$ BTU $/ 1 \mathrm{bm}$

$\dot{M} 13=585 \mathrm{lbm} / \mathrm{Hr}$

$\dot{\mathrm{V}} 13=3380 \mathrm{Ft}^{3} / \mathrm{Hr}$

SATURATED VAPOR

HATER 


\section{A QUOTATION FROM}

THE PFAUOLER CO.

DIVISION OF SYBRON CORPORATION
TO: Department of Mechanical Engineering Auburn University Auburn, AL 36830

Attention: Mr. Paul Chancellor
QUOTATION NO. H-9455-A

DATE

OFFICE

PAYMENT

F.O.B.
October 11,1979

Rochester, NY

Net 15 days from date of invoice

Elyria, $\mathrm{OH}$

WE ARE PLEASED TO QUOTE THE FOLLOWING:

REFERENCE

QUANT ITY

DESCRIPTION
Item A.

One (1) Condenser.

Pfaudler Model 16192 BEM vertical, one pass Heat Exchanger with (115) 3/4" diameter 18 BWG tubes on $1^{\prime \prime}$ triangular pitch.

Tubes are $16 \mathrm{ft}$. long in 316 stainless steel yielding $361 \mathrm{sq}$. ft. of heat transfer surface.

This unit has a $16^{\text {". }}$ diameter, one pass, carbon steel shell with one carbon steel $1^{\prime \prime}$ diameter nozzle and one carbon steel $2^{\prime \prime}$ diameter nozzle.

The shell is $m$ ated to one long 316 stainless steel bonnet and one short 316 stainless steel bonnet complete with compressed asbestos gaskets, two 316 stainless steel 2 " diameter nuzzles.

She 11 side 150 psig at $375^{\circ} \mathrm{F}$.

Tube side 300 psig at $375^{\circ} \mathrm{F}$.

Add $\$ 130.00$ for ASME Code Stamp.

2,700165 .

F.0.B. Elyria, Ohio, (each).....\$10,122.00.

12 to 14 weeks after receipt of an order following settling of all details.

HE MHINTED TEAMS AND CONDITIONS ON THE BAGX HEREOF AAE PART OF THIS QUOTATION. BUYEA'S PURCHASE ORDEA SHALL CONSTITUTE AN OFFER TO SELLER.

SUBMITTED BY

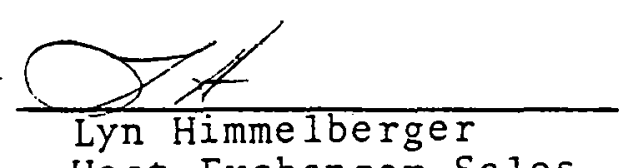

Heat Exchanger Sales

(Continued on page ............. attached, which constitutes part of this ouotation.) 


\subsection{6}

QUOTATION/CONTINUED

Department of Mechanical Engineering

SEAL OPTION

ALTERNATE

TERMS \& CONDITIONS
Pfaudler has the capability of using two separate techniques for the tube-to-tubesheet joint: the roller expansion method and the strength welding technique. Both of these are avialable at the same price. A schematic of the strength welded design for fixed tubesheet tube bundles is avail$a b l e$ upon request. Please advise up on placement of an order which technique you would prefer. If you do not specify a preference, we will utilize the strength welded technique.

For an expansion joint, add.....\$678.00. Also, shell side pressure rating is 75 psig. Delivery will require four (4) additional weeks.

Our terms and conditions are on the back of Sheet No. 1 of this quotation. Any variance from terms and conditions on the reverse side of the quotation will have no force or effect unless negotiated by both parties and consented. to in writing.

THE PFAUDLER COMPANY A division Of SYBRON CORPORATION

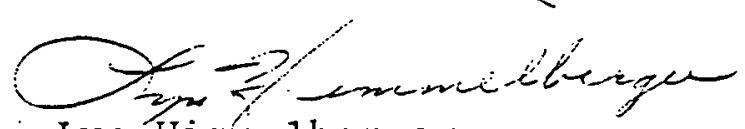

Lyn Himme lberger

Heat Exchanger Sales

LH: jmt

Enc1. 


\section{The Expansion Valves}

Three pneumatically controlled expansion valves are required for the demonstration unit construction. They are an integral part of the control system design and therefore must be.compatable with other control components and circuits. The valves specified by the control system designers were quoted as separate items by the Foxboro Company. The quotations received are shown in the following pages. 


\section{QUOTATION}

To Auburn University

Dept. of Mechanical Eng.

Auburn, Alabama 36830

ATTENTION OF Mr. Thomas Burch

\author{
No. \\ 2-1-283- Additional \\ Please refer to this \\ number when ordering \\ DATE January 23, 1980 \\ PLEASE SEND YOUR \\ PURCHASE ORDER TO: \\ 3393 Malone Drive \\ Chamblee, Ga. 30341
}

YOUR INQUIRY FOXboro Order 80D-0001

Remarks :

This offering is for the Foxboro Company offering

Categories and standard conditions of sale attached.

Orders placed on or before the expiration date shown

below will be bllied at the prices quoted.

F.O.B. Foxboro, Mass.

TERMS: Full Payment 30 days from dates of invoices.

ESTIMATED SHIPMENT: 12-16 weeks

THIS QUOTATION EXPIRES: FEb. 24, 1980

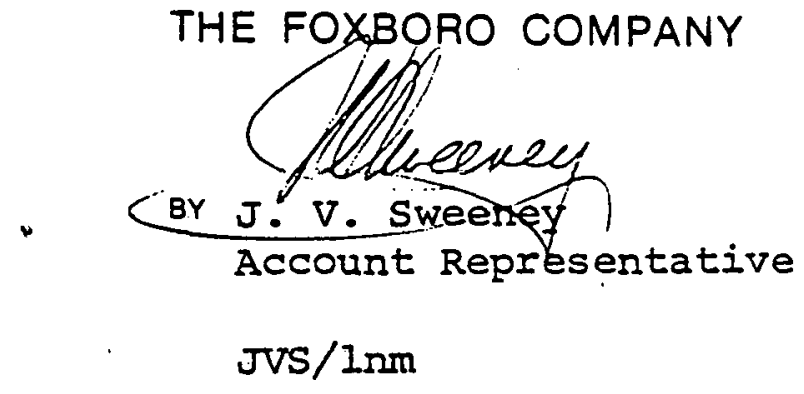


TRIM SIZE AND

CHARACTERISTIC :

ACTUATOR :

SUPPIY AND ACTION: OPTIONA工 FEATURES:

INPUT SIGNAT :

ACTION :

REQUIRED DATA

SERVICE :
Carbon steel

Flanged, ANSI Class 300

(Steel body) Standard bolted bonnet, ptfe guide bushing, 316 ss stem,

17-4PH plug and seat, ptfe $Y$ rings packing.

Equal Percentage Cv0.5 reduced port P25D

20 psi, air-to-open Pneumatic Positioner, Type $C$ with plain manifold $(\mathrm{V}-\mathrm{CO})$. 3-15 psig Increasing input, inc. output.

Liquid Methonal

PRICE EACH: 
QUOTAIION FROM THE FOXBORO COMPANY

\section{Auburn University}

Auburn, Alabama

$5.40 \quad 2-1-283$

No.Add' 1 .

SHEET

3 DATE

$1 / 23 / 80$

aPATUS HEREIN ENUMERATED WILL BE FURNISMED UNOEA THE CONOMONS PRINTED IN FORM 2547 - OFFEAING CATEGORIES AND STANOARO CONDITIONS OF SALE

\begin{tabular}{|c|c|c|c|}
\hline TEM & QUAN & & \\
\hline 7 & 1 & $\begin{array}{l}\text { FOXBORO BUTTERFLY CONTROI } \\
\text { MODEL: } \\
\text { NOMINAI SIZE: } \\
\text { BODY MATERIAI: } \\
\text { END CONNECTIONS : } \\
\text { CONSTRUCTION : } \\
\text { ACTUATOR : } \\
\text { VALVE ACTION: } \\
\text { SIGNAL RANGE: } \\
\text { OPTIONAL FEATURES : } \\
\text { REQUIRED DATA } \\
\text { SERVICE : } \\
\text { TAG: } \\
\text { I4 InCh Valve and Flanges } \\
\text { I6 InCh VaIVe and Flanges }\end{array}$ & $\begin{array}{l}\text { VAIVE } \\
\text { S2FY-2YC } \\
16 \text { in } \\
\text { Cast Iron } \\
\text { Flanged } \\
\text { Cast Iron Body and Trim, } \\
\text { Flanged ends, ball bearing } \\
\text { shaft, max working pressure } \\
15 \text { psig. } \\
\text { Model p50 } \\
\text { Air-to-open } \\
3 \text { to I5 psig } \\
1 \text { pair companion flanges } \\
\text { Air Bleed } \\
\text { Air Bleed } \\
\text {-PRICE: } \\
\text {-PRICE: }\end{array}$ \\
\hline
\end{tabular}

$\$ 1328.00$

$\$ 1635.00$ 


\section{The Control System}

The actual control system design is presented in chapter 6 of this manual. The components specified by the control system and quoted by the Foxboro Company are shown in the following pages for completeness. 


\section{QUOTATION}

To Auburn University

Dept. of Mechanical Engineering

Auburn, Alabama 36830

ATTENTION OF' Mr. Thomas Burch
NO. $\frac{2-1-283}{\begin{array}{c}\text { Please refer to this } \\ \text { number when ordering }\end{array}}$

DATE January 14, 1980

PLEASE SEND YOUR PURCHASE ORDER TO:

3393 Malone Drive Chamblee, Ga. 30341

YOUR INQUIRY FOXbOrO Order 80D-0001

Remarks :

This offering is for The Foxboro Company offering

Categories and Standard Conditions: of sale attached.

$$
+0+21 \$ 3120
$$

Orders placed on or betore the explratiun date shown

below will be billed at the prices quoted.

F.O.B. Foxboro, Mass.

TERMS: Full Payment 30 days from dates of invoices.

ESTIMATED SHIPMENT: 10-12 weeks

THIS QUOTATION EXPIRES: FEbruarY 15, 1980

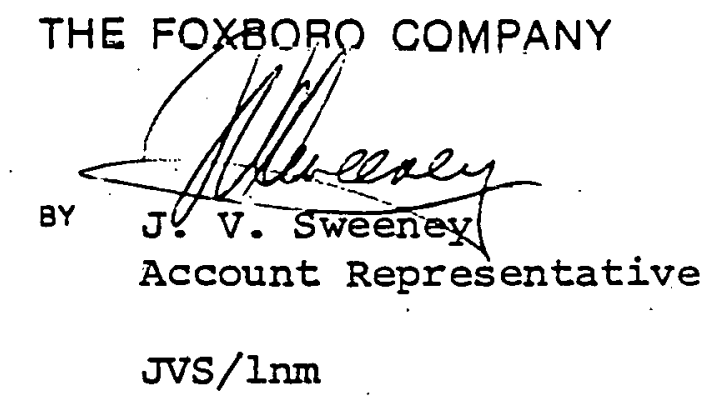


RANGE :

PRICE : 
Auburn University

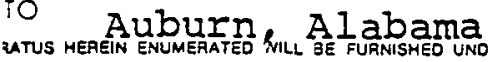

$$
\text { No. } 2-1-283 \text { SheEt } 3 \text { Date } 1 / 14 / 80
$$

OTIONS PAINTED IN FORM 25AT - OFFEFING CATEGORIES AND STANOAAO CONOITIONS CF SALE

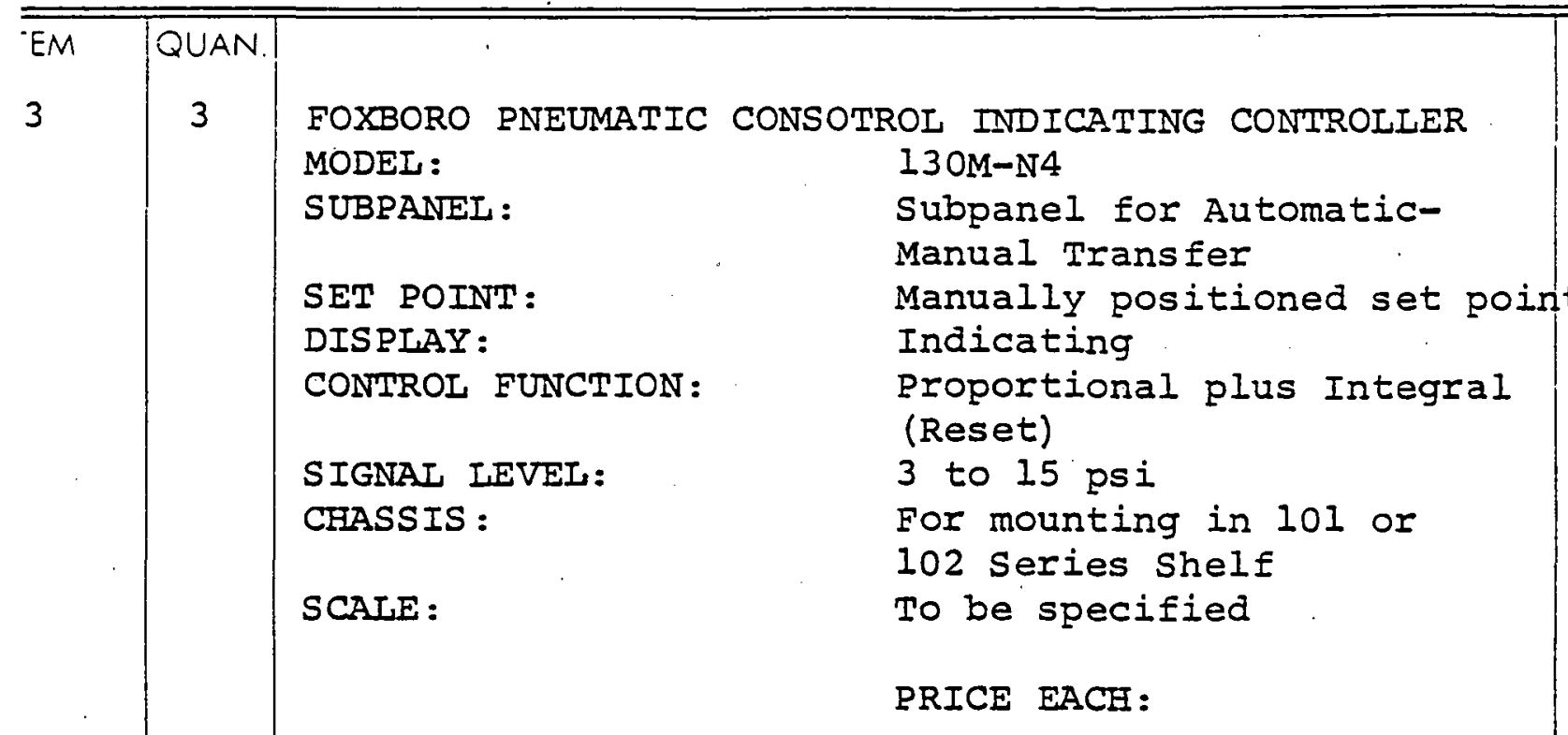

DIFFERENTIAL DEVIATION PRESSURE SWITCH

MODEL :

Barksdale Model DPD2T-A3

PRICE :

1 FOXBORO PNEUMATIC CONSOTROL SHELF

MODEL :

SHELF SIZE :

CONNECTIONS :

SHELF ARRANGEMENT:

Shelf

Position

1

2

3
$102-3 \mathrm{~N}$

3 units of mounting capacity Back of shelf Pneumatic, 1/4 NPT

Sequence is from left to right facing front of shelf Shelf conn. Set Type Number 102-CO $102-\mathrm{CO}$ 102-CO

PRICE :
$\$ 183.00$

$\$ 909.00$

$\$ 279.00$ 


\section{Chapter 6}

\section{THE CONTROL SYSTEM}

Chapter four discussed the three independent variables which determine system performance. They are:

T3A - exit temperature of compressor's second stage,

Tl - the evaporator's tube side inlet temperature,

T6 - the evaporator's shell side exit temperature.

The control system's job is to maintain these three variables at 01 near the optimum set points in addition to providing boiler feedwater.

Figure 6.1 shows a schematic of the waste heat recovery system with integral controis. Each of the various control loops are referenced and function as shown in the table below.

Table 6.1

\begin{tabular}{|c|c|}
\hline Control Loop & Function \\
\hline 1. Zones $A$ ? and $A 2$ & $\begin{array}{l}\text { Maintain an energy balance by monitoring dryer } \\
\text { hood exhaust temperature and modulating exhaust } \\
\text { bleed valve. }\end{array}$ \\
\hline 2. Zone B & $\begin{array}{l}\text { Protect the compressor from liquid by monitoring } \\
\text { the differential pressure across } H X E \text { and modu- } \\
\text { lating the valve between accumulator tank } \frac{\|}{\pi 2} \\
\text { and } H X E \text {. }\end{array}$ \\
\hline 3. Zone $c$ & $\begin{array}{l}\text { Maintain a fixed refrigerant mass flow rate by } \\
\text { monitoring the liquid level in accumulator tank } \\
\# 2 \text { and modulating the valve between accumulator } \\
\text { tank } \# 2 \text { and } H X C \text {. }\end{array}$ \\
\hline 4. Zone $D$ & $\begin{array}{l}\text { Maintain boiler feedwater by monitoring the water } \\
\text { level in HXA and cycling the condensate pump. }\end{array}$ \\
\hline
\end{tabular}




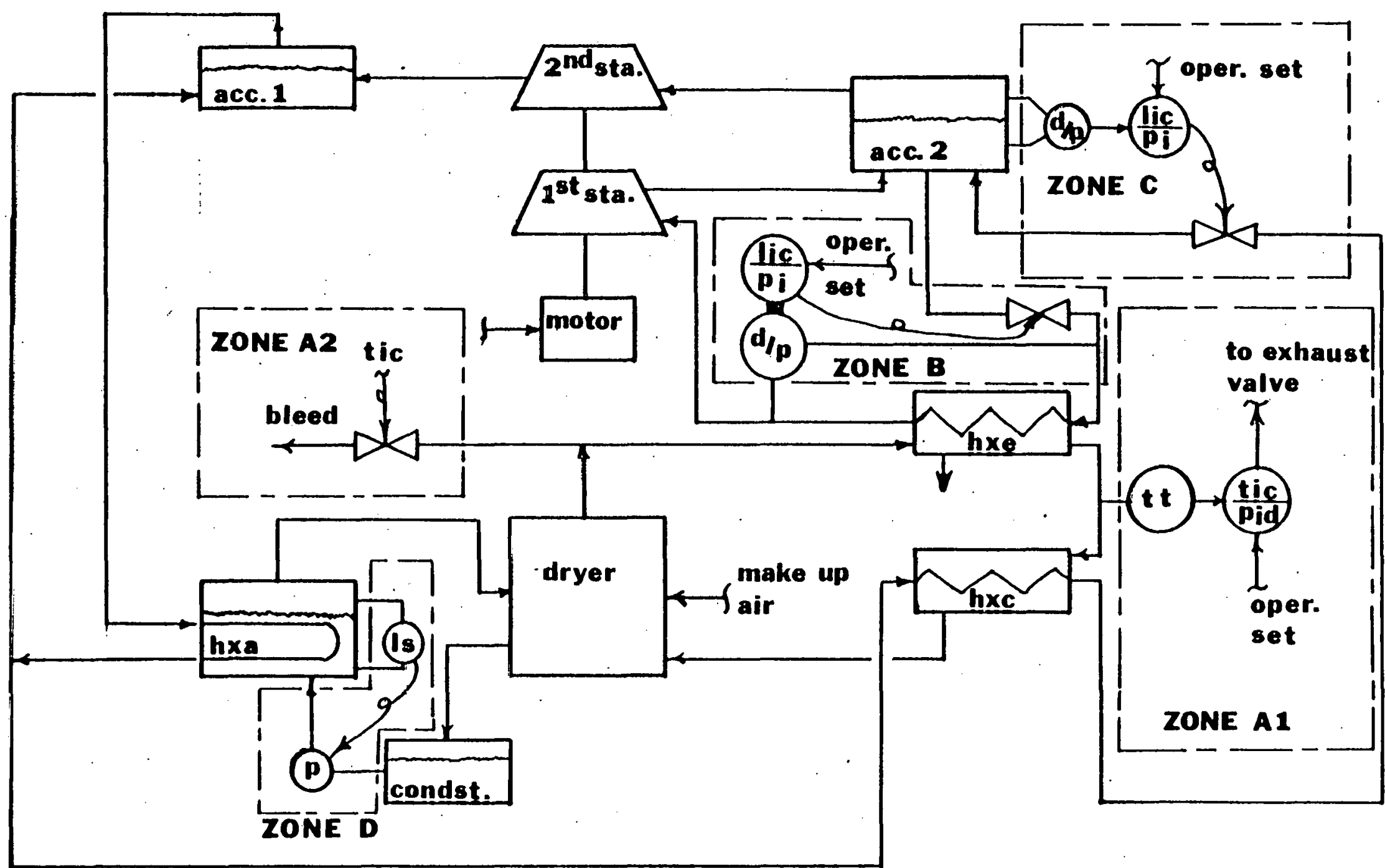

FIGURE 6.1

CONTROL SYSTEM 


\section{3}

The components required to implement this control system are shown in Table 6.2 along with their mode of control.

Table 6.2

\begin{tabular}{|l|l|l|}
\hline Zone & Components & Mode of Control \\
\hline \multirow{3}{*}{ Al } & $\begin{array}{l}\text { Temperature Transmitter } \\
\text { Temperature Indicating } \\
\text { Controller }\end{array}$ & $\begin{array}{l}\text { Proportional } \\
\text { Integral } \\
\text { Differential }\end{array}$ \\
B & $\begin{array}{l}\text { Differential Pressure Cell } \\
\text { Level Indicating Control }\end{array}$ & $\begin{array}{l}\text { Proportional } \\
\text { Integral } \\
\text { C }\end{array}$ \\
Differential Pressure Cel1 \\
Level Indicating Control & $\begin{array}{l}\text { Proportional } \\
\text { Integral } \\
\text { Deffon }\end{array}$ \\
\hline
\end{tabular}

Detailed specifications for these components are included in Chapter 6 of this report. 


\section{Chapter 7 \\ LAYOUT AND CONSTRUCTION}

Assembly of the demonstration model is facilitated greatly since most of its components are free-standing. Figure $7: 1$ gives an isometric view of the system component arrangement along with the piping and ductwork needed. Line sizing was performed by the computer program and validated by hand calculation as shown in Table 7.1 below (see Figure 7.2 for line designations).

TABLE 7.1

\begin{tabular}{|l|c|}
\hline Line Designation & $\begin{array}{c}\text { Line Diameter } \\
\text { (in inches) }\end{array}$ \\
\hline Disat & 5 \\
\hline DISH & 5 \\
\hline$D_{3}$ & 4 \\
\hline$D_{3} \mathrm{~A}$ & 3 \\
\hline$D_{3} \mathrm{~B}$ & 2 \\
\hline$D_{4}$ & 2 \\
\hline$D_{4} \mathrm{~A}$ & 2 \\
\hline$D_{2}$ & 6 \\
\hline
\end{tabular}




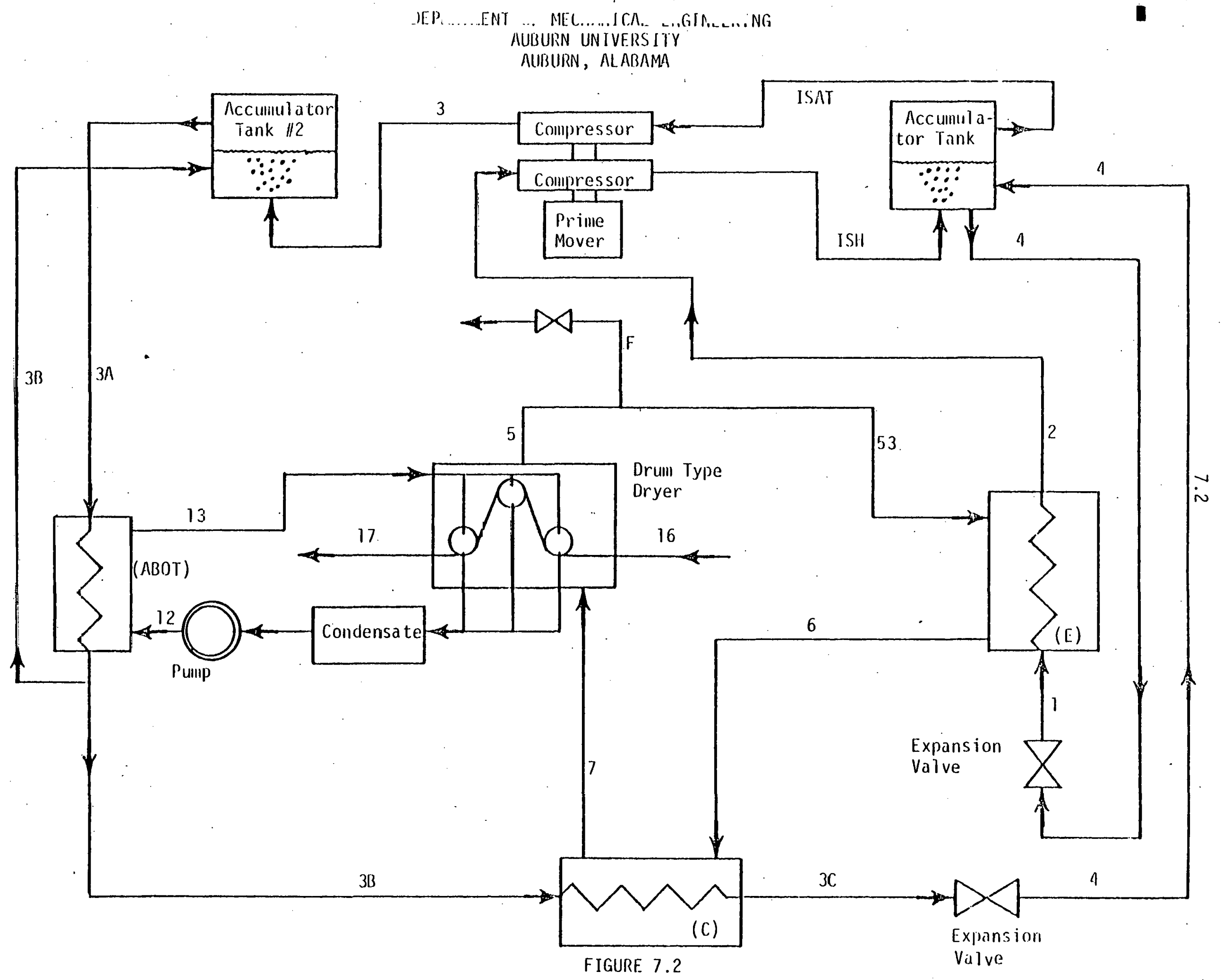




\section{ISOMETRIC DRAWING OF PROTOTYPE SYSTEM}

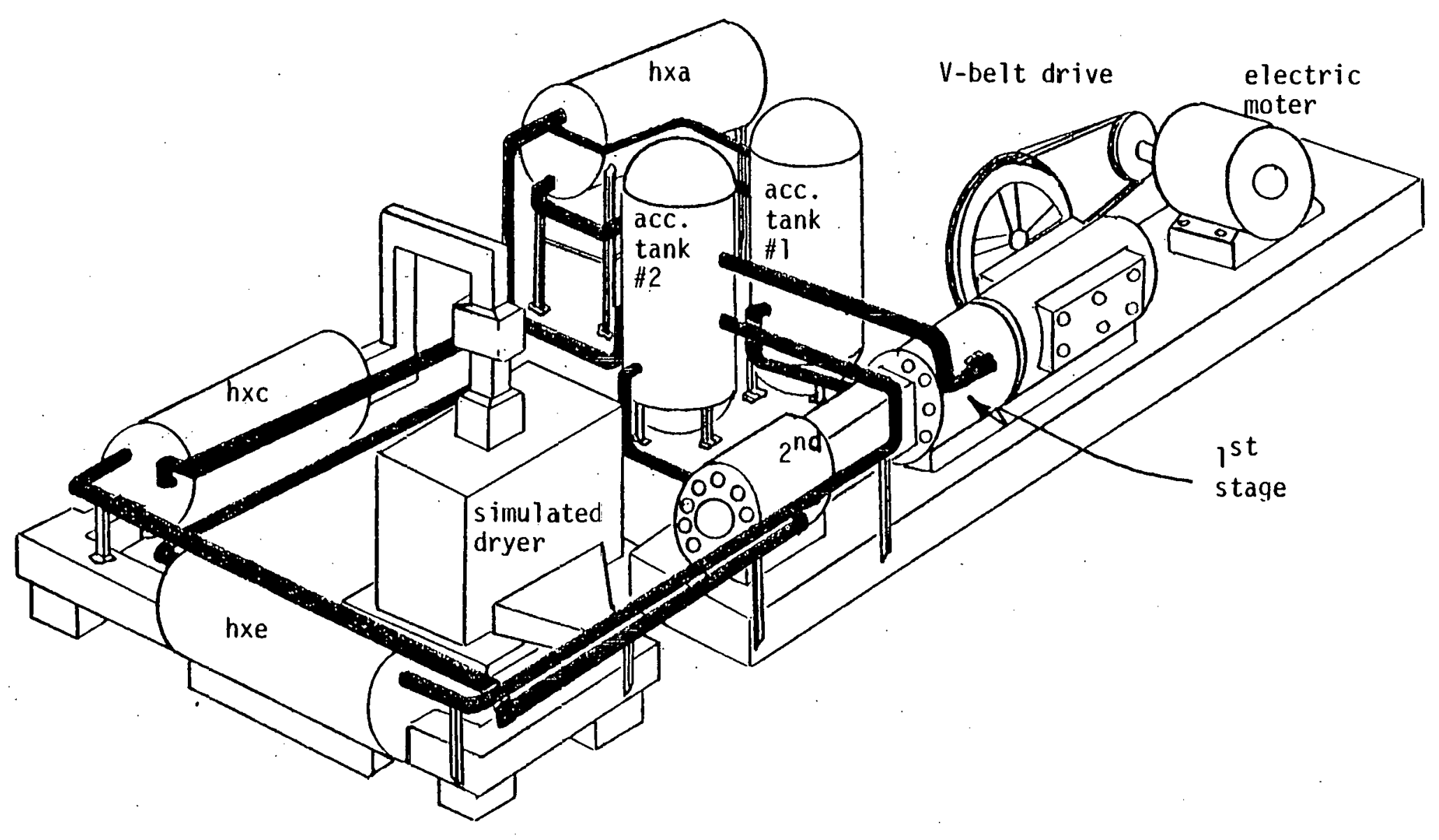


TOP VIEW OF PROTOTYPE SYSTEM LAYOUT

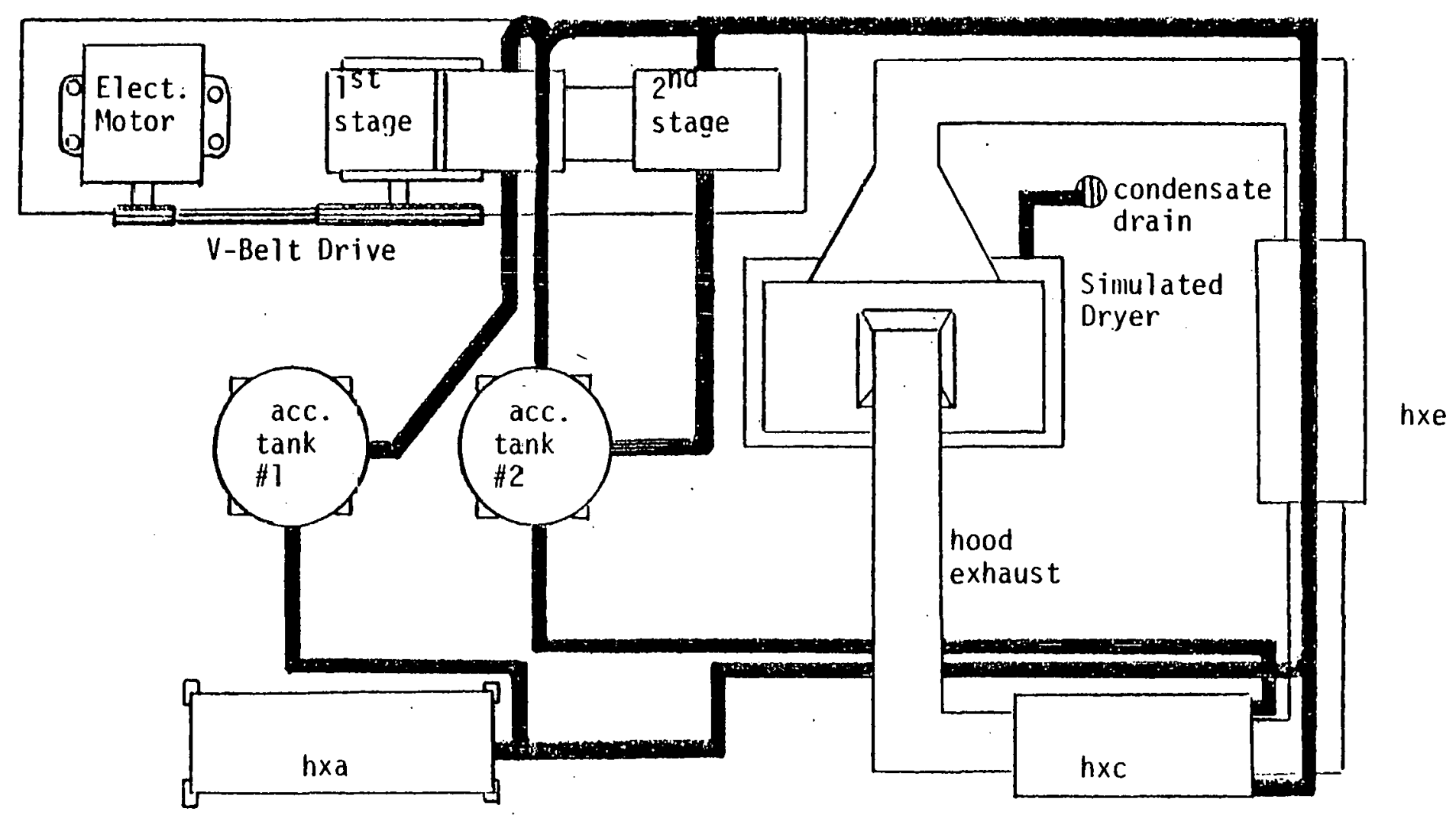

FIGURE 7.3 
The objectives of the system layout are:

1. To provide for ease of access

2. To allow adequate safety precautions

3. To minimize line lengths where high heat transfer rates might be a problem

4. To fit into the allocated space

Figure 8.3 illustrates a top view of the system layout and reflects the objectives stated above. 


\section{Chapter 8}

\section{ECONOMICS AND SUMMARY}

Phase I of this contract has been completed within the allotted time and budget. Each of the deliverable items is included within this final report excepting only the Operators Manual which is bound separately.

The work accomplished in Phase I represents considerably more effort than was originally anticipated as can be seen by the graph below (Figure 8.1).

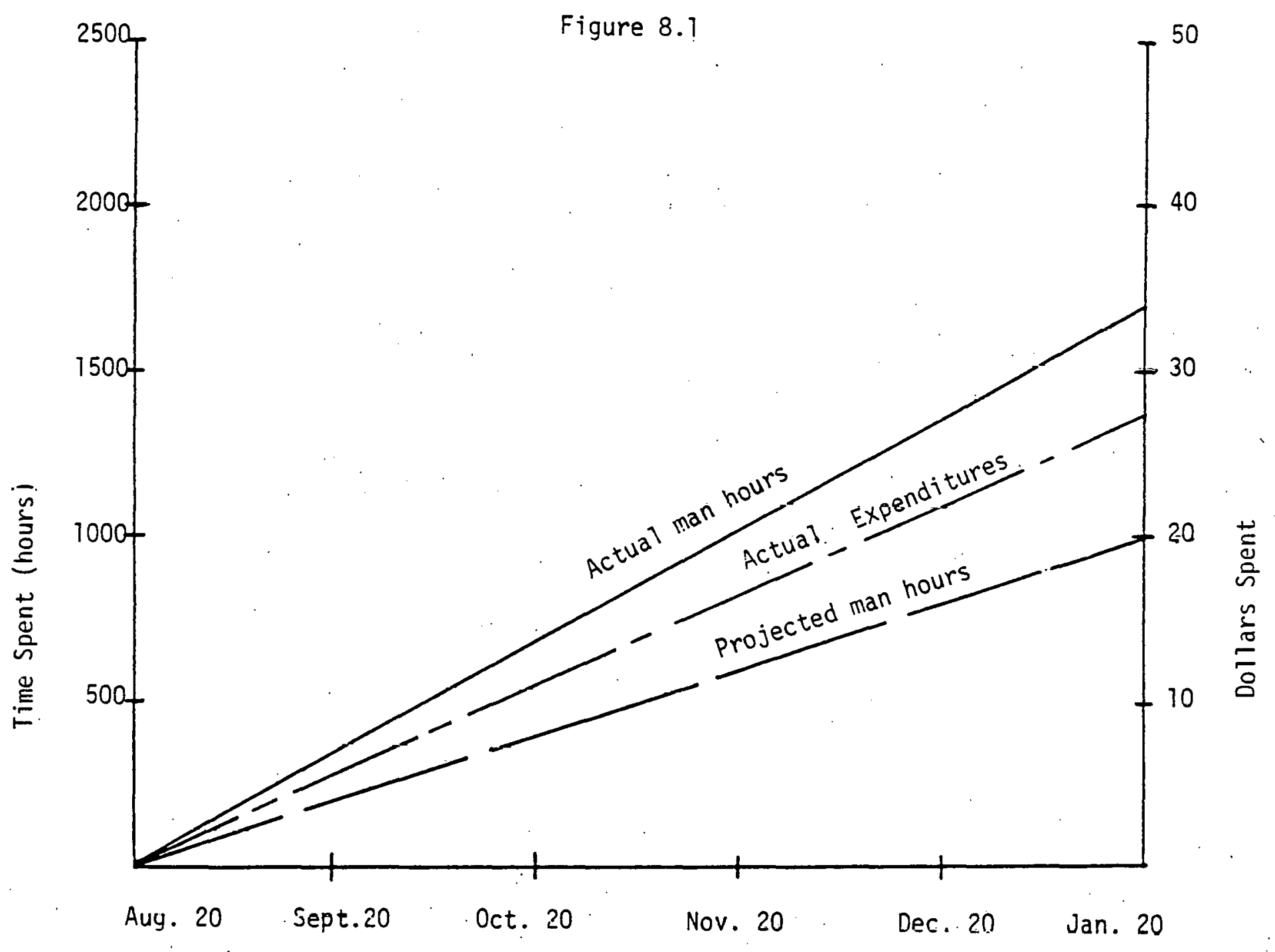

Analysis of Phase I Time and Expense 
The increased man hours required reflect that time required to select a more appropriate working fluid, extend the thermodynamic data for that fluid, and provide the consequent engineering information necessary for specifying system configuration and components. This additional work, some 700 man hours, has been performed in order to deliver the items specified in Phase I of the contract; the Department of Energy has incurred no additional charges for this work.

Since each of the three phases and their budgets were included in the original proposal to DOE, it is evident that a revised budget is needed to insure adequate funds for the completion of Phase II. The table below (Figure 8.2) includes the proposed budget and the revised budget which reflects increases in both man hours and component costs.

Figure 8.2

\begin{tabular}{|l|c|c|}
\hline \multicolumn{1}{|c|}{ Category } & Proposed $\$$ & Actual \$ \\
\hline Salaries and Wages & 75,377 & 38,377 \\
System Components & 26,015 & 94,469 \\
Miscellaneous & 10,717 & 21,434 \\
Inflation & -- & 1,240 \\
\hline TOTALS & $\$ 62,109$ & $\$ 155,520$ \\
\hline
\end{tabular}


Figure 8.3 further divides the system component category to explain the significant cost deviation from that proposed.

Figure 8.3

\begin{tabular}{|l|c|c|c|}
\hline \multicolumn{1}{|c|}{ Component } & Proposed Cost $\$$ & Actual Cost $\$$ & Difference \\
\hline $\begin{array}{l}\text { Compressor } \\
\text { with Motor } \\
\text { Heat Exchangers } \\
\text { Controls and } \\
\text { Instrumentation }\end{array}$ & 11,550 & 55,800 & 44,250 \\
Valves & 2,970 & 18,220 & 15,250 \\
Ducts \& Piping & 10,505 & 13,705 & 3,200 \\
Inflation D 10\% & 590 & 2,504 & 1,512 \\
\hline TOTALS & 550 & 3,550 & 3,000 \\
\hline
\end{tabular}

As can be seen from Figure 8.3, the most outstanding difference from proposed costs and actual costs is the compressor cost. The reasons for this high compressor cost are:

1. Using methanol as a working fluid.

2. Building a model rather than a full size unit.

Since methanol is the working fluid; a two-stage compression is required. This essentially doubles compressor cost per horsepower. Also, methanol temperature approaches $400^{\circ} \mathrm{F}$ at the exit from the second stage. This high temperature would be no problem for a large centrifugal compressor (e.g., > 100 HP), but poses a serious problem for smaller reciprocating compressors. Non-standard design features are required to alleviate the problems of operating a reciprocating compressor at these high temperatures. The result is a much higher cost per compressor horsepower. 


\section{4}

Realizing the disproportionate cost of the compressor for the $50 \mathrm{HP}$ demonstration model, the investigators have sought for viable alternatives. If the demonstration system were scaled up from $50 \mathrm{HP}$ to $100 \mathrm{HP}$, it may be possible to use a rotary screw compressor supplied by Frick and Company. Since the time and budget of Phase I was limited, it has been impossible to perform a detailed evaluation of this alternative. Assuming that a 100 HP rotary screw compressor could be supplied by Frick and Company, the budget for building such a system is presented below in Figure 8.4.

Figure 8.4

\begin{tabular}{|l|c|}
\hline \multicolumn{1}{|c|}{ Category } & Projected Cost \$ \\
\hline System Components & $\vdots$ \\
Salaries and Wages & 100,258 \\
Inflation @ i0\% & 38,066 \\
Miscellaneous & 2,480 \\
\hline TOTAL & 21,434 \\
\hline
\end{tabular}

